\title{
IPHAS and the symbiotic stars
}

\section{Selection method and first discoveries $\star \star \star \star$}

\author{
R. L. M. Corradi 1,2, E. R. Rodríguez-Flores ${ }^{2,3}$, A. Mampaso ${ }^{2}$, R. Greimel ${ }^{1}$, K. Viironen ${ }^{2}$, \\ J. E. Drew ${ }^{4,5}$, D. J. Lennon ${ }^{1,2}$, J. Mikolajewska ${ }^{6}$, L. Sabin ${ }^{2}$, and J. L. Sokoloski ${ }^{7}$ \\ 1 Isaac Newton Group, PO Ap. de Correos 321, 38700 Sta. Cruz de la Palma, Spain \\ e-mail: rcorradi@ing.iac.es \\ 2 Instituto de Astrofísica de Canarias, 38200 La Laguna, Tenerife, Spain \\ 3 Instituto de Geofísica y Astronomía, Calle 212, N. 2906, CP 11600, La Habana, Cuba \\ ${ }^{4}$ Imperial College of Science, Technology and Medicine, Blackett Laboratory, Exhibition Road, London, SW7 2AZ, UK \\ 5 Centre for Astrophysics Research, STRI, University of Hertfordshire, College Lane, Hatfield, AL10 9AB, UK \\ ${ }^{6}$ N. Copernicus Astronomical Center, Bartycka 18, 00-716 Warsaw, Poland \\ 7 Columbia Astrophysics Laboratory, USA
}

Received 4 November 2007 / Accepted 10 December 2007

\begin{abstract}
Context. The study of symbiotic stars is essential to understand important aspects of stellar evolution in interacting binaries. Their observed population in the Galaxy is however poorly known, and is one to three orders of magnitudes smaller than the predicted population size.

Aims. IPHAS, the INT Photometric $\mathrm{H} \alpha$ survey of the Northern Galactic plane, gives us the opportunity to make a systematic, complete search for symbiotic stars in a magnitude-limited volume, and discover a significant number of new systems.

Methods. A method of selecting candidate symbiotic stars by combining IPHAS and near-IR (2MASS) colours is presented. It allows us to distinguish symbiotic binaries from normal stars and most of the other types of $\mathrm{H} \alpha$ emission line stars in the Galaxy. The only exception are T Tauri stars, which can however be recognized because of their concentration in star forming regions.

Results. Using these selection criteria, we discuss the classification of a list of 4338 IPHAS stars with H $\alpha$ in emission. 1500 to 2000 of them are likely to be Be stars. Among the remaining objects, 1183 fulfill our photometric constraints to be considered candidate symbiotic stars. The spectroscopic confirmation of three of these objects, which are the first new symbiotic stars discovered by IPHAS, proves the potential of the survey and selection method.
\end{abstract}

Key words. surveys - Galaxy: stellar content - stars: binaries: symbiotic - stars: emission-line, Be - stars: pre-main sequence ISM: planetary nebulae: general

\section{Introduction}

Symbiotic stars are the interacting binaries with the longest orbital periods. They are composed of a compact star, in most cases a hot white dwarf, accreting from the wind of a cool giant companion. Part of the giant's wind is ionized by the white dwarf, producing the composite spectrum containing both absorption features from a cool stellar photosphere and emission lines from highly excited ions: these characteristics originally caused these objects to be named "symbiotic". Depending on their near-IR colours, symbiotic stars are divided into "stellar" (S) types, if colours are typical of red giant branch (RGB) stars,

\footnotetext{
* Based on observations obtained at the $2.5 \mathrm{~m}$ INT telescope of the Isaac Newton Group of Telescopes in the Spanish Observatorio del Roque de Los Muchachos of the Instituto de Astrofísica de Canarias. This publication makes use of data products from the Two Micron All Sky Survey, which is a joint project of the University of Massachusetts and the Infrared Processing and Analysis Center/California Institute of Technology, funded by the National Aeronautics and Space Administration and the National Science Foundation. This research has also made use of the SIMBAD database, operated at CDS, Strasbourg, France.

$\star \star$ Table 1 is only available in electronic form at

http://www. aanda.org
}

or "dusty" (D) types, if their near-IR emission shows a significant contribution from the warm dust known to be typical of evolved asymptotic giant branch (AGB) stars. S-types account for around $80 \%$ of the sample of known symbiotic stars. The presence of an RGB or an AGB star also determines the orbital separation at which the symbiotic phenomenon occurs; orbital periods for the S-types are between 200 and 6000 days, while they are longer for the D-types ( $>20 \mathrm{yr}$, but no single robust determination exists so far). For more details on the properties of symbiotic stars, see e.g. Corradi et al. (2003).

A variety of phenomena occur in symbiotic stars that are relevant to a number of important astrophysical problems. For example, symbiotic stars have been proposed as potential supernova Ia progenitors (Munari \& Renzini 1992; Hachisu et al. 1999). Also, symbiotic stars are excellent laboratories for studying (i) thermonuclear outbursts (nova-like accretion instabilities) under a wide range of conditions (cf. Munari 1997); (ii) the powering mechanism of supersoft X-ray sources (cf. Jordan et al. 1996); (iii) the collimation of stellar winds and the formation of jets (cf. Tomov 2003) and (iv) bipolar (planetary) nebulae (cf. Corradi 2003).

A crucial figure in the discussion of some of these topics is the total number of symbiotic stars in the Galaxy. This is 
basically unknown. In fact, no systematic search for symbiotic stars in the Milky Way has been done so far, and the present sample of 173 known Galactic systems (Belczyński et al. 2000), plus another 26 suspected ones, is mainly the result of occasional discoveries during the study of peculiar, variable or erupting stars, or of sparse objective prism surveys. This figure should be compared with the predicted total number of symbiotic stars in the Galaxy, which spans two orders of magnitude: $3 \times 10^{3}$ (Allen 1984), $3 \times 10^{4}$ (Kenyon et al. 1993), $3 \times 10^{5}$ (Munari \& Renzini 1992), and $4 \times 10^{5}$ (Magrini et al. 2003).

IPHAS, the INT Photometric H $\alpha$ survey of the Northern Galactic plane (Drew et al. 2005), gives us the opportunity to improve the determination of this basic number. The search for symbiotic systems in IPHAS takes advantage of the generally strong $\mathrm{H} \alpha$ emission that characterizes this class of objects. In this paper, we define our criteria for the detection of symbiotic stars using the data from the IPHAS project, complemented by near-IR colours from the 2MASS survey. The effectiveness of the method is illustrated by the spectroscopic confirmation of three new symbiotic stars.

\section{The data}

\subsection{IPHAS}

IPHAS is an international collaboration, whose aim is to produce a complete, fully photometric, and spatially detailed $\mathrm{H} \alpha$ map of the part of the Galactic Plane between latitudes $-5^{\circ}$ and $+5^{\circ}$ that is visible from the Northern hemisphere. The IPHAS observations are obtained using the Wide Field Camera (WFC) at the prime focus of the $2.5 \mathrm{~m}$ Isaac Newton Telescope (INT) on La Palma, Spain. The WFC consists of a mosaic of four $2 \mathrm{k} \times 4 \mathrm{k}$ EEV CCDs, providing a field of view of $34 \times 34 \operatorname{arcmin}^{2}$ with a sampling of 0.33 per pixel. The IPHAS images are taken through three filters: a narrow-band $\mathrm{H} \alpha\left(\lambda_{\mathrm{c}}=6568 \AA ; F W H M=95 \AA\right)$ and two broad-band Sloan $r$ and $i$ filters, with matched 120 , 30 , and $10 \mathrm{~s}$ exposures, respectively. In this way, the magnitude range $13 \leq r \leq 20$ is covered for point sources (the fainter end at $10 \sigma)$. Pipeline data reduction and data distribution are handled by the Cambridge Astronomical Survey Unit. The presentation of the survey and further details can be found in Drew et al. (2005).

At the time of writing this article, more than $90 \%$ of the $\sim 1800$ square degrees of the northern Galactic Plane to be covered by IPHAS has been observed. A first photometric catalogue, containing more than 200 million objects, is about to be released (González-Solares et al. 2008). From it, a list of $4853 \mathrm{H} \alpha$ emitting stars with $r<19.5$ mag has been extracted by Witham et al. (2008). This is the sample that we consider in this paper for our first search for symbiotic stars within IPHAS. Independently, we are employing other methods to select both point-like and extended $\mathrm{H} \alpha$ emitters from the IPHAS observations (see e.g. Viironen et al. 2008): we will consider these additional samples in following papers (note that most symbiotics have a stellar profile, but a small fraction of them might be extended in the $\mathrm{H} \alpha$ filter due to their resolved nebulosity, see Corradi 2003).

\subsection{Reference samples of known objects}

Unsaturated IPHAS data are available for only four known symbiotic stars from the general catalogue of Belczyński et al. (2000). They are DQ Ser, V352 Aql, V1413 Aql, and Ap 3-1. In order to build a meaningful reference sample of known symbiotic stars to be used to define our selection method, additional observations were obtained at the INT using the same instrumental setup as for IPHAS. Photometric data of 18 known symbiotic stars were taken on June 2, 2004, and April 20, and September 17 and 18, 2005. Exposure times were tuned so as to avoid saturation of the (generally bright) targets. Reduction was done with IRAF. Moreover, existing flux-calibrated spectra of 18 symbiotic stars (one of which is in common with the sample above), obtained at different epochs, were convolved with the WFC filters and instrument response curves to derive $\mathrm{H} \alpha, r$ and $i$ magnitudes in the IPHAS photometric system. Where the $i$ magnitude cannot be derived from the spectra, we adopted the $r-i$ colour from Munari et al. (1992). All together, we collected a sample of 39 known symbiotic stars (29 of the $S$ type and 10 of the D type) with magnitudes and colours fully consistent with those produced by the photometric catalogue of IPHAS. This constitutes the primary reference sample to develop our selection method.

We have also investigated other classes of stars and nebulae which can potentially be confused with symbiotic stars according to their IPHAS colours, because of also having $\mathrm{H} \alpha$ in emission, or because their spectral type is similar to that of the cool component of symbiotic stars. Using IPHAS and additional observations, we have derived $\mathrm{H} \alpha, r$ and $i$ magnitudes for 67 planetary nebulae (PNe, Viironen et al. 2008), 79 cataclysmic variables (Witham et al. 2006), and 518 Mira variables. As Be stars are a frequent class of $\mathrm{H} \alpha$ emitting bright stars in the Galactic plane (cf. Sect. 4.1), they deserve special attention: 18 of these stars in the low-reddening open clusters NGC 663, NGC 869 and NGC 884, as well as 22 objects in the more reddened cluster NGC 7419, turned out to have good IPHAS photometric data and were adopted as the comparison samples for this class of stars. Another frequent class of $\mathrm{H} \alpha$ emitters in star-forming regions are T Tauri stars; their characteristics derived from IPHAS data on Cyg OB2 are being investigated by Vink et al. (2008). Finally, $\mathrm{H} \alpha$ emission is also observed in late $\mathrm{K}$ to $\mathrm{M}$ dwarfs with enhanced chromospheric and coronal activity (dMe stars). However, their $\mathrm{H} \alpha$ emission is generally faint, with line equivalent widths ${ }^{1}$ of a few $\AA$ (Hawley et al. 1996). These are much smaller than in symbiotic stars (see Sect. 4.3), and for this reason $\mathrm{dMe}$ stars are not further considered in this paper.

\subsection{Near-IR 2MASS magnitudes}

As symbiotic stars contain a luminous cool giant, near-IR magnitudes provide information both on their nature and support making distinctions between them and other classes of objects. The division in the two main groups of $\mathrm{S}$ and $\mathrm{D}$ types was indeed originally made using the $J-H$ and $H-K$ colours (Allen \& Glass 1974). Recently, the position of symbiotic stars in the near-IR colour-colour diagram was discussed by Rodríguez-Flores (2006) and Phillips (2007).

We have therefore extracted from the Two Micron All Sky Survey (2MASS) database the $J, H$ and $K_{\mathrm{S}}$ magnitudes of 187 known symbiotic stars, 288 PNe (most of them from Ramos-Larios \& Phillips 2005), 95 cataclysmic variables, 1230 Be stars (1148 of which are from Zhang et al. 2005), 487 T Tauri stars (Dahm \& Simon 2005), and 121 Mira variables (Rodríguez-Flores 2006).

\footnotetext{
1 According to the standard definition, the equivalent width of an emission line would be negative. However, in order to simplify the discussion, in this paper we use a positive sign, so that a larger $\mathrm{H} \alpha$ equivalent width corresponds to a stronger $\mathrm{H} \alpha$ emission, and to a larger $r-\mathrm{H} \alpha$ colour.
} 


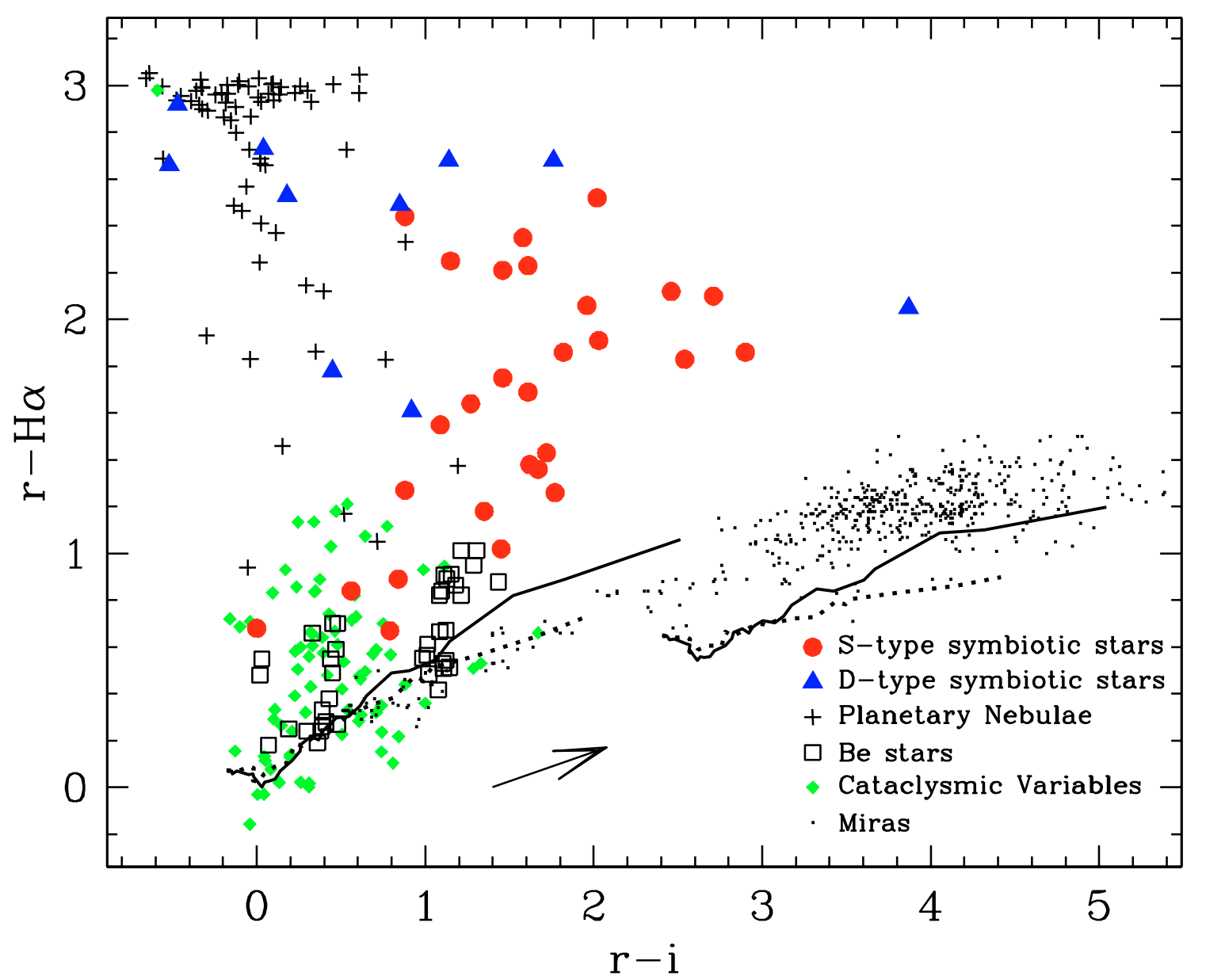

Fig. 1. IPHAS colour-colour diagram for different classes of objects. The locus of main-sequence and RGB stars is indicated by the solid and dotted lines, respectively. Two sequences are shown, corresponding to reddening values $E(B-V)=0$ (left) and 4 (right), respectively. The arrow indicates the reddening vector for normal stars: its length corresponds to 3 mag extinction in $V$.

Similarly, we have searched for 2MASS counterparts of the $4853 \mathrm{H} \alpha$ emitters in Witham et al. (2008). For 4330 of them, we found a 2 MASS source within 1 arcsec from the IPHAS coordinates (note that the two sets of data are calibrated into the same astrometric system). Also, the 2MASS counterpart was identified for another 8 objects with slightly worse astrometric match. This makes a total sample of $4338 \mathrm{H} \alpha$ emitters with 2MASS magnitudes.

It should be noted that the detection limits of IPHAS ( $r \leq$ 19.5 mag for the objects in Witham et al. 2008) and of the 2MASS point-source catalogue $\left(K_{\mathrm{S}} \sim 15 \mathrm{mag}\right)$ roughly match the characteristic colours of symbiotic stars. We have considered a list of 71 known and bright symbiotic stars $(r \leq 14.5 \mathrm{mag})$ : $70 \%$ of them have indeed an observed optical-to-near-IR colour $\left(r-K_{\mathrm{S}}\right) \geq 4.5$. On the average, they are presumably closer and less reddened than the new symbiotics stars that we aim to detect with IPHAS, for which we then expect to be able to find a 2MASS counterpart. We are therefore confident that adding 2MASS data does not affect the completeness of our search for symbiotic stars within IPHAS.

\subsection{Follow-up spectroscopy}

As the present study was progressing, we started a campaign of spectroscopic follow-up of the $\mathrm{H} \alpha$ emitters detected by IPHAS. Accordingly a dozen candidate symbiotic stars, selected as described in the next sections, were observed at the INT using the IDS spectrograph, on nights of May 11, June 14, and September 9, 2006. Grating R300V was used, which gives a reciprocal dispersion of $1.87 \AA$ per pixel of the $2 \mathrm{k} \times 4 \mathrm{k}$ EEV detector, and a spectral coverage from 3800 to $8500 \AA$ (these figures slightly vary from night to night). The slit width was 1.1 arcsec projected on the sky, providing a spectral resolution of $5 \AA$. Exposure times were $30 \mathrm{~min}$ for the two brighter sources discussed in Sect. 5, and $2 \mathrm{~h}$ for the faintest and more reddened one. Several spectrophotometric standards were observed during the night for relative flux calibration. Reduction was performed using the package onedspec in IRAF. Note that the EEV CCD suffers from significant fringing redward of $\sim 7000 \AA$, which was not possible to remove with the calibration frames obtained during those nights. Also, the flux calibration is somewhat uncertain above $8000 \AA$ because of significant optical aberrations at the edge of the large format CCD used with IDS.

\section{Interpreting the IPHAS and 2MASS colour-colour diagrams}

\subsection{The IPHAS colour-colour diagram}

Our primary tool for the selection of candidate symbiotic stars in the Galactic Plane is the IPHAS $r-\mathrm{H} \alpha$ vs. $r-i$ colour-colour diagram, which is presented and thoroughly discussed in Drew et al. (2005). It is shown in Fig. 1 for the various classes of objects considered. In the diagram, the $r-\mathrm{H} \alpha$ axis mainly indicates 


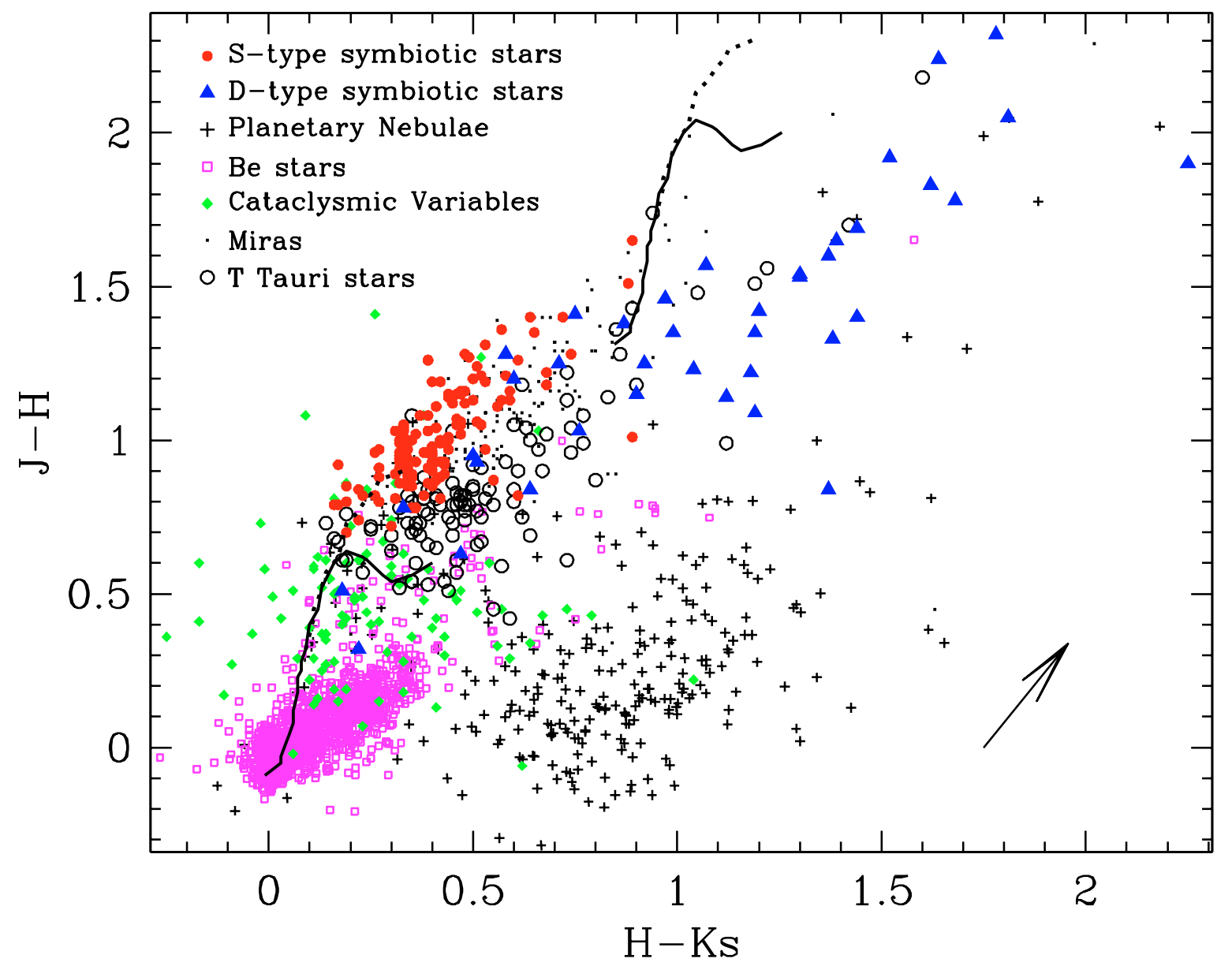

Fig. 2. 2MASS colour-colour diagram for different classes of objects. Symbols are the same as in Fig. 1, albeit smaller owing to the larger number of objects. In addition, T Tauri stars are indicated by empty circles. Like in Fig. 1, the locus of main-sequence and RGB stars is indicated by the solid and dotted lines, respectively, for reddening values $E(B-V)=0$ (lower-left sequence) and 4 (upper-right sequence). The arrow shows the reddening vector for normal stars corresponding to 3 mag extinction in $V$.

increasing values of the $\mathrm{H} \alpha$ emission line equivalent width, while $r-i$, for normal stars, is essentially a sequence of increasing spectral types and/or reddening. In the figure, the locus of main-sequence and RGB stars is indicated by the solid and dotted lines, respectively (Drew et al. 2005). Two sequences are shown, corresponding to reddening values $E(B-V)=0$ (left) and 4 (right), respectively. The reddening vector for normal stars, adopted from Howarth (1983) in the same way as by Drew et al. (2005), is indicated by the arrow: its length corresponds to 3 mag extinction in $V$. We note that, as shown by Drew et al. (2005), at the higher extinctions probed by IPHAS $\left(6<A_{V}<12\right.$, roughly) this vector flattens progressively and in a mildly spectral-type dependent manner.

The positions of the different classes of object are indicated as follows. Symbiotic stars of the $\mathrm{S}$ type are shown as filled circles, and the D types as triangles. In the case that several measurements of the same object obtained at different epochs are available, we plot the mean colours as the dispersion of symbiotic stars in the graph is anyway large enough to cover their intrinsic variability. PNe are indicated by crosses; cataclysmic variables by filled diamonds; Be stars by empty squares; Mira variables by dots. In all cases, we show the observed colours.

As expected, given their emission-line spectra are superposed on relatively weak continua, PNe stand out in the graph for their extreme $r-\mathrm{H} \alpha$ colours. Most of them lie close to the $r-\mathrm{H} \alpha \sim 3.1$ limit which is expected for pure $\mathrm{H} \alpha$ emission line stars ("ideal" sources for which all the flux in the $\mathrm{H} \alpha$ and $r$ bands comes from the $\mathrm{H} \alpha$ line). With smaller $\mathrm{H} \alpha$ excesses than $\mathrm{PNe}$ (with some overlap), but still above the other classes of $\mathrm{H} \alpha$ emitters and normal stars, lie the symbiotic stars. They span a significant range in $r-i$ colour. Be stars and cataclysmic variables are located closer to the locus of main-sequence stars with some moderate $\mathrm{H} \alpha$ excess (except for Nova Aql 1995, which is now in the nebular phase and shows a large $r-\mathrm{H} \alpha$ colour). Mira variables (which only sometimes show the $\mathrm{H} \alpha$ line in emission) are mainly found at the right of the diagram, owing to their cool photosphere and frequent large reddening by circumstellar dust. The class of T Tauri stars is not shown in the figure, as they are spread all over the diagram above the sequence of normal stars (e.g. Vink et al. 2008). They show a broad range of spectral types as well as $\mathrm{H} \alpha$ equivalent widths. The latter are in most cases smaller than $500 \AA$ (see Fig. 5 in Dahm \& Simon 2005), which is lower than for the most extreme symbiotic stars. Our strategy to separate them, at least in a statistical sense, from the evolved stars on which we are focusing our attention in this work, will be presented in Sect. 4.4.

The symbiotic stars with the smallest $r-\mathrm{H} \alpha$ colours belong to the rarer subgroup of the yellow symbiotics, containing G-K giants. Examples are BD-213873, AG Dra and LT Del. Also, the figure suggests that the D-types have $r-\mathrm{H} \alpha$ colours generally larger than the S-types, and more similar to PNe. This was already remarked upon in the past (cf. Kenyon 1986), and in fact 
the distinction between PNe and some D-type symbiotic stars is tricky (cf. Corradi 2003). However, our present sample is limited to ten objects.

Further information on the location of symbiotic stars in the IPHAS diagram, albeit limited to the $r-i$ colour, can be obtained from the literature. Munari et al. (1992) published $R$ and $I$ magnitudes for a large sample of symbiotic stars. We have transformed these data to the Sloan system for the INT+WFC, and in this case we are able to correct for interstellar extinction using the data in Whitelock \& Munari (1992). The resultant range of intrinsic $r-i$ colours spanned by 56 symbiotic stars of $\mathrm{S}$ type is between -0.1 and $+2.8 \mathrm{mag}$, while the range spanned by 19 D-type systems is even larger, from -0.7 to +3.6 mag.

\subsection{The $2 M A S S$ colour-colour diagram}

The previous section has shown the potential of the IPHAS colour-colour diagram to separate symbiotic stars from the vast majority of stars. However, some overlap is present with classes of $\mathrm{H} \alpha$ sources whose population in the Galactic Plane is much larger than that of symbiotic stars. In addition, mixing between classes is inevitably raised in the presence of photometric errors.

The 2 MASS $J-H$ vs. $H-K_{\mathrm{S}}$ colour-colour diagram provides an additional resource to refine the selection of symbiotic stars (Rodríguez-Flores 2006). The various classes of objects, as well as normal main-sequence and giant stars (Bessel \& Brett 1988), are indicated in Fig. 2 with the same symbols as in Fig. 1. In addition, we plot here as empty circles 104 classical T Tauri stars from Dahm \& Simon (2005) with an $\mathrm{H} \alpha$ equivalent width larger than $40 \AA$ (roughly corresponding to the smallest $\mathrm{H} \alpha$ equivalent width shown by the known symbiotic stars in Sect. 3.1). All these $\mathrm{T}$ Tauri stars belong to the young cluster NGC 2264, which has a low foreground extinction $\left(A_{V}=0.22 \mathrm{mag}\right)$, and is rich in pre-main sequence $\mathrm{H} \alpha$ emitters (Dahm \& Simon 2005). For all objects we plot the observed colours. As for the IPHAS diagram, the reddening vector is computed from Howarth (1983) for the 2MASS filters $J, H$, and $K_{\mathrm{s}}$ : the adopted shifts for a reddening of $1 \mathrm{mag}$ in $V$ are 0.113 and 0.069 for the $J-H$ and $H-K_{\mathrm{S}}$ colours, respectively.

The bulk of PNe occupy a distinct locus at the bottom of the near-IR diagram (see also Ramos-Larios \& Phillips 2005), to the right of normal stars. Most of the cataclysmic variables and Be stars are instead clumped closer to the locus of main-sequence stars. In particular, note the well-defined sequence at the bottom of the diagram displayed by Be stars, which extends to the right side of the unreddened main-sequence stars. We will come back to this important feature in Sect. 4.1.

S-type symbiotic stars, according to their original definition (Allen \& Glass 1974), have colours typical of RGB stars, with an upper-right tail due to reddening. They form a compact cluster in the diagram, except for few objects. The most extreme case is UV Aur (a carbon star), which is displaced to the right of the S-types sequence, toward the broad region which is occupied by $\mathrm{T}$ Tauri stars, D-type symbiotic stars, and some extreme Mira variables. The location of D-type symbiotics is modeled by Phillips (2007) with a variable combination of stellar and warm dust continuum emission, plus some extinction. Three objects, AS 201, V471 Per, and StHA 190, have significantly smaller $J-H$ colours than the bulk of symbiotics. Again, these are yellow symbiotics, which tend to occupy the bottom part of the locus of symbiotic stars, as in the IPHAS diagram.

Some PNe fall in the region of D-type symbiotic stars. These are examples of possible misclassified PNe, which might
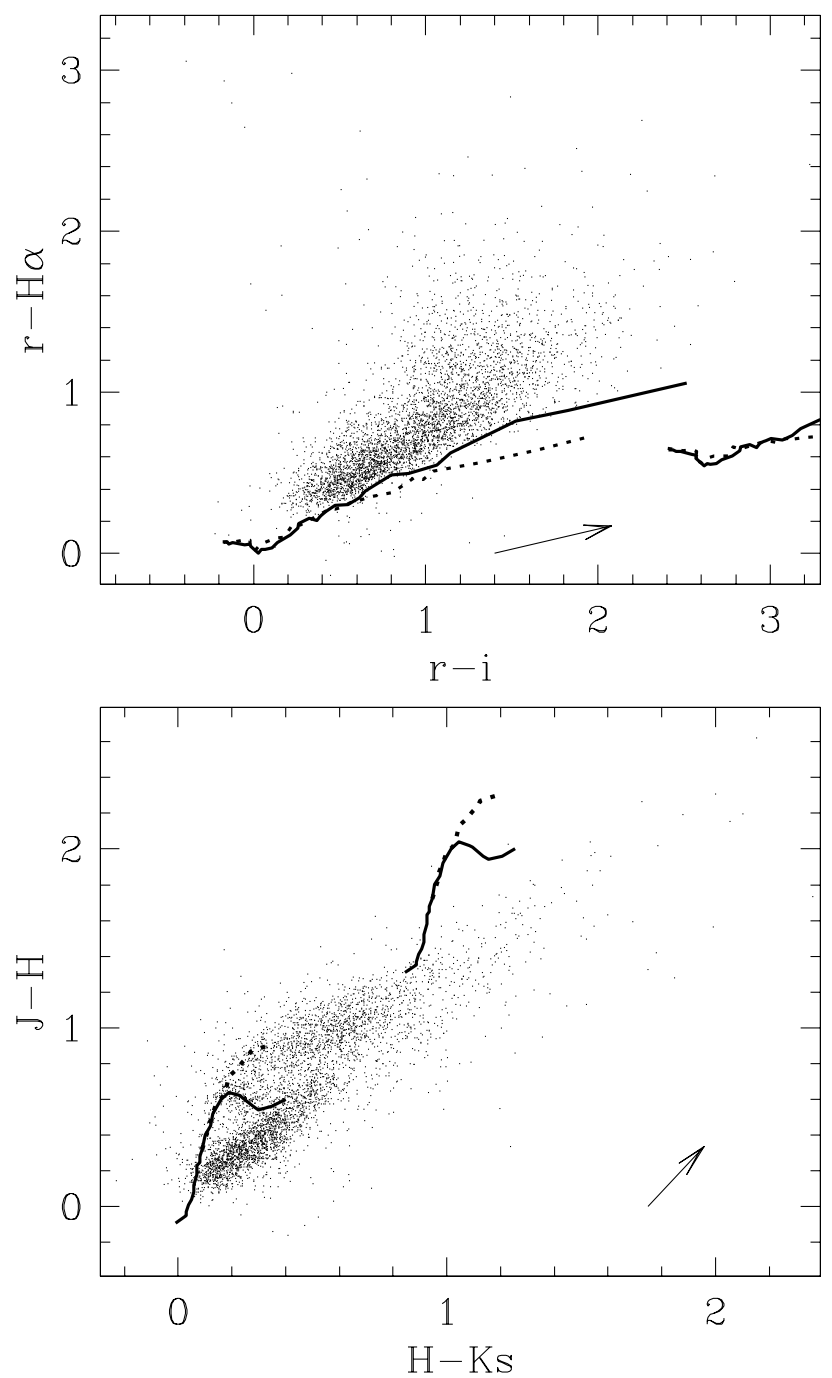

Fig. 3. The $4338 \mathrm{H} \alpha$ emitters by Witham et al. (2008) in the IPHAS (top) and 2MASS (bottom) colour-colour diagrams. The loci of mainsequence and giant stars and the reddening vectors are also indicated (see Fig. 1 for a detailed explanation).

instead be symbiotic stars with extended nebulae as frequently suggested in the literature (Corradi 1995; Schmeja \& Kimeswenger 2001; Santander-García et al. 2007).

Finally, T Tauri stars form a broad sequence running parallel to, and on right side of, S-type symbiotics, that extends well into the locus of the D-type symbiotic stars. Note that T Tauri stars with smaller equivalent widths than those displayed, including the so-called weak-line T Tauri stars, would be clumped on the left-bottom end of this sequence (Dahm \& Simon 2005). The significant overlap with symbiotic stars in both the 2MASS and IPHAS diagrams, makes T Tauri stars the most frequent "contaminants" in our search for symbiotics stars.

\section{Application to the list of IPHAS $\mathrm{H} \alpha$ emitters by Witham et al. (2008)}

The analysis of the IPHAS and 2MASS colour-colour diagrams presented in the previous section allows us to discuss the nature of the 4338 IPHAS $\mathrm{H} \alpha$ emitters by Witham et al. (2008) with 2MASS counterparts (Sects. 2.1 and 2.3). Their location in the colour-colour diagrams is shown in Fig. 3. Looking at the 2MASS diagram, there are some striking similarities with 

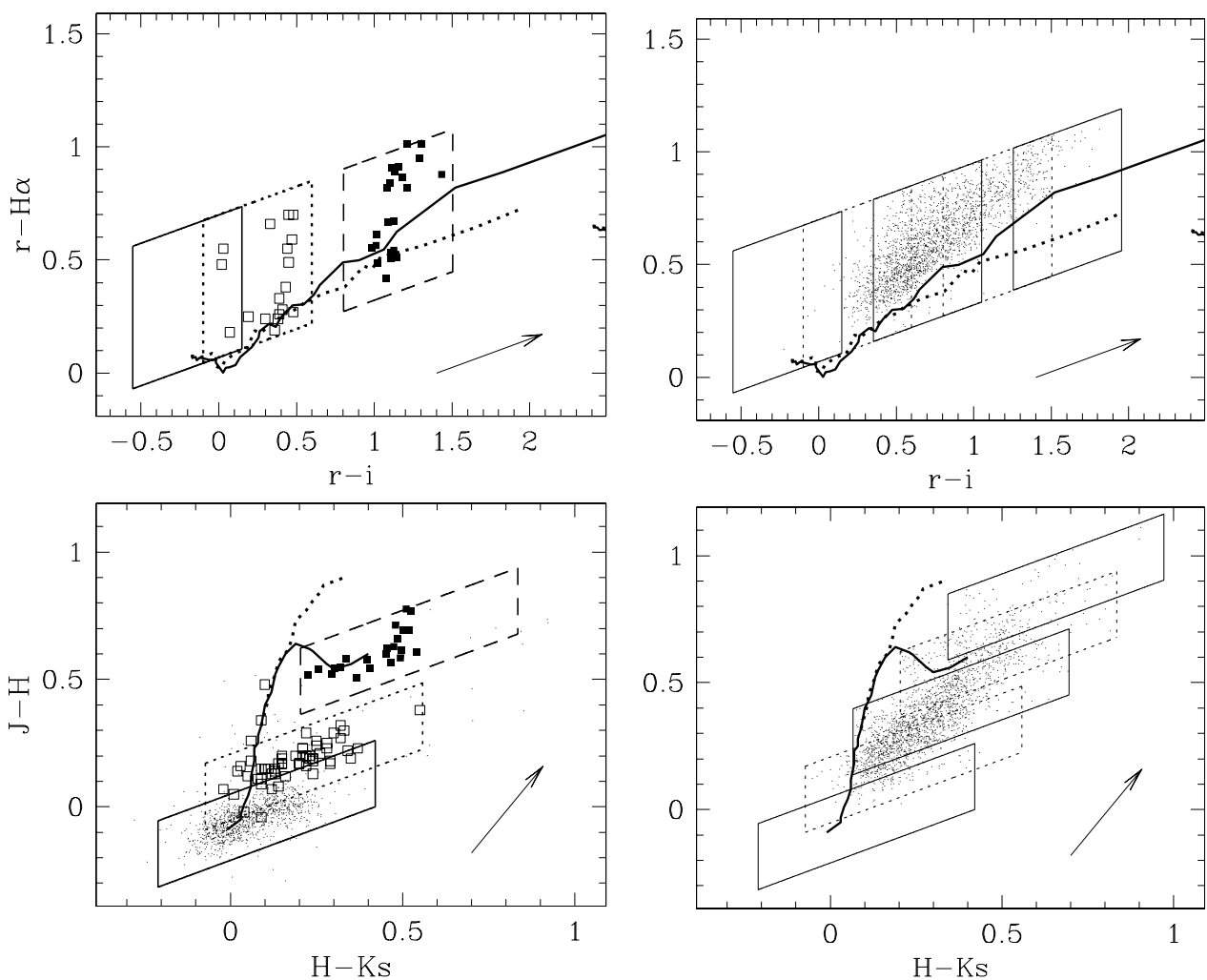

Fig. 4. Left: location of known Be stars in the IPHAS and 2MASS diagrams. The dots mark dereddened colours for the 1148 Be stars from Zhang et al. (2005). Open squares are the reddened data for the open clusters NGC 663, NGC 869 and NGC 884, and filled squares similar data for NGC 7419. The solid line is the adopted zero-reddening selection box for Be stars. Right: the 2035 candidate Be stars in the list of $4338 \mathrm{H} \alpha$ emitters by Witham et al. (2008). The alternate solid/dotted boxes show the selection boxes (see text) for $A_{\mathrm{v}}=0,2,4$, 6, $8 \mathrm{mag}$, from bottom-left to top-right, respectively. the plot of known objects in Fig. 2. First, the 2MASS diagram of Fig. 3 shows a main concentration of sources at its bottom, to the right of the unreddened main-sequence stars. They form a dense band with a slightly lower inclination than our adopted reddening vector. This is obviously similar to the sequence defined by known Be stars in Fig. 2. Second, another inclined sequence of objects can be seen in Fig. 3 at higher $J-H$ values, in the region where $\mathrm{T}$ Tauri stars (frequent in the Galactic Plane) and symbiotic stars (presumably less numerous) should be located.

We therefore start our discussion by considering what appears to be one of the most represented classes of objects in the list of Witham et al. (2008): the Be stars.

\subsection{Be stars}

In order to further investigate the similarities between Fig. 2 and 3, we have studied in more detail the location of Be stars in the colour-colour diagrams. Zhang et al. (2005) provide the reddening of 1148 individual objects: this allow us to identify the locus of unreddened Be stars in the 2MASS diagram, and to define a corresponding "selection" box. These are indicated by the dots and the solid line, respectively, in the lower-left panel of Fig. 4. When shifted to the reddening of the clusters NGC 663, NGC 869 and NGC 884 ( $A_{V} \sim 2$ mag, dotted line) and NGC 7419 ( $A_{V} \sim 6$ mag, dashed line), such a box includes the corresponding data points for these clusters (empty and filled squares, respectively).

Similar consistent results are obtained for the IPHAS colourcolour diagram. There, if we define the locus of Be stars from the position of the objects in the four open clusters considered, and correct for the corresponding reddening, we can determine a zero-reddening selection box for this class of objects, which is shown as a solid line in the upper-left panel of Fig. 4.

We have then done the exercise of extracting candidate Be stars from the 4338 IPHAS H $\alpha$ emitters in Witham et al. (2008).
An object is considered to be a good candidate Be star if it falls inside the selection boxes in both the IPHAS and the 2MASS diagrams. The combination of the selection boxes in the two diagrams, shifted so as to consider a range of reddening values from $A_{V}=0$ to $A_{V}=8 \mathrm{mag}$ (represented by the sequence of boxes in the right panels of Fig. 4), results in a total of 2035 candidate Be stars (also plotted in Fig. 4). The majority of them would have reddening values between 3 and 5 mag in $V$. Objects at zero reddening are virtually absent, presumably because such objects are in the main nearby and thus bright and saturated in IPHAS. Only for significant reddening values (i.e. at the largest $J-H$ colours, cf. the lower-right panel of Fig. 4 with Fig. 2), mixing of the Be stars candidates with low-reddening $\mathrm{T}$ Tauri stars is possible. An idea of the magnitude of the contamination can be gained considering that only $4 \%$ of the $450 \mathrm{~T}$ Tauri stars in Dahm \& Simon (2005), including those with the lowest $\mathrm{H} \alpha$ equivalent widths, have $J-H \leq 0.55$. Adopting this figure, and the worst possible (and unlikely) scenario that all sources with $J-H>0.55$ in our complete list of 4338 objects are T Tauri stars, it would be concluded that only one hundred objects of this class would be expected with a lower $J-H$ colour, at variance with the more than 1600 Be star candidates ( $80 \%$ of our list) lying below this colour limit.

A smaller amount of mixing is expected with other, less frequent classes of objects, like CVs, compact PNe, and symbiotic stars. We conclude that somewhere between 1500 and 2000 Ho emitters in Witham et al. (2008) are likely to be Be stars, which would therefore be the largest class of objects in this list of IPHAS H $\alpha$ stars.

\subsection{Planetary nebulae}

In Sect. 3.1, we remarked on the overlap in the IPHAS diagram between PNe and symbiotic stars, especially those of the D-type. On the other hand, the 2MASS colours are generally different, 

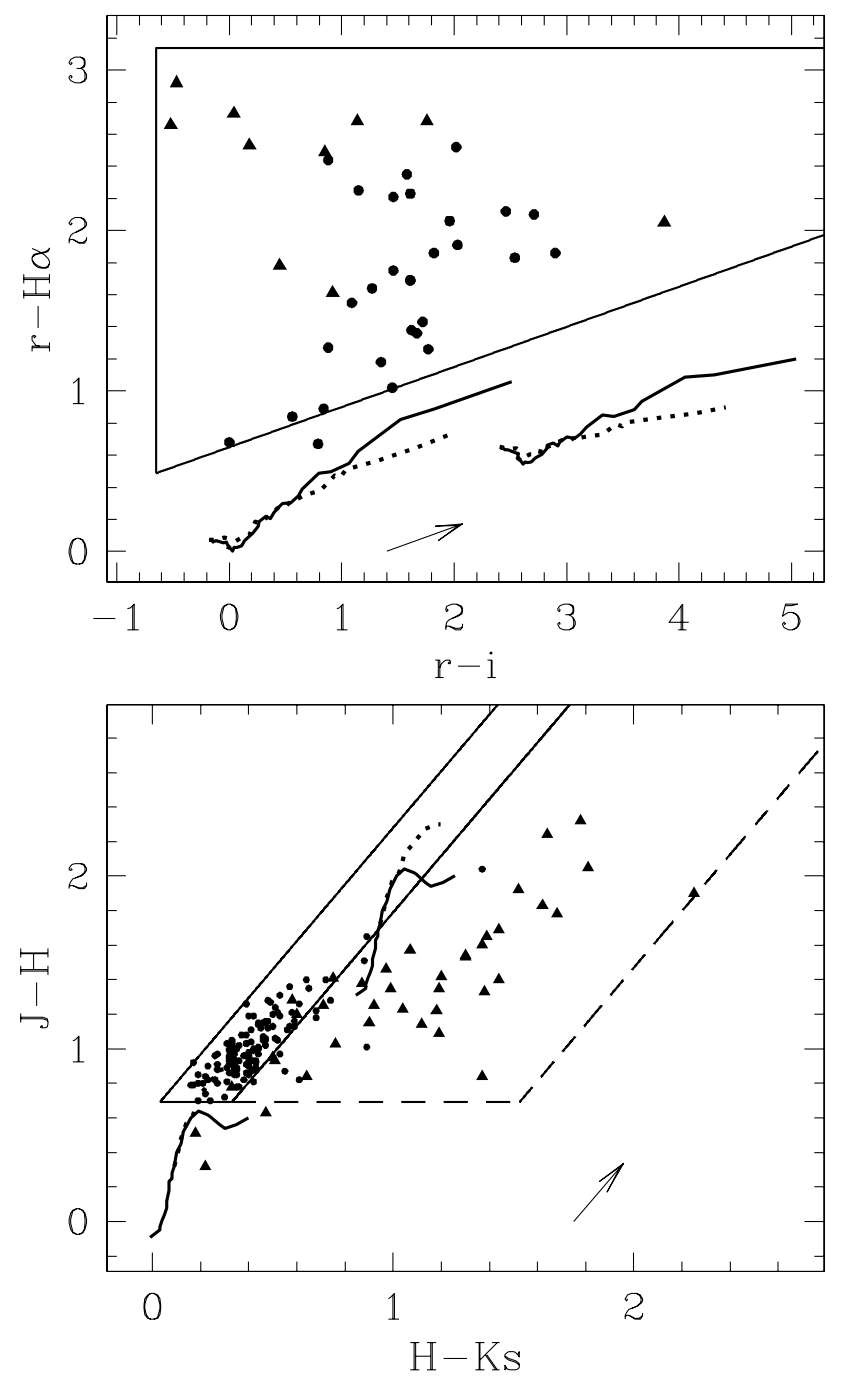

Fig. 5. Top: the selection box for symbiotic stars in the IPHAS colourcolour diagram, superimposed on the reference sample and the locus of main-sequence and RGB stars (see Fig. 1 for details). Bottom: the selection boxes for the S-type (continuous line) and the D-type (dashed line) symbiotics in the 2MASS diagram. Symbols as in Fig. 2.

allowing a neat separation of the two classes of objects (with notable exceptions which might be related to misclassification). A search for compact PNe from the list of Witham et al. (2008), resulting in a few new candidates, and a confirmation of their nature by spectroscopic observations, is presented elsewhere (Viironen et al. 2008).

\subsection{Symbiotic stars}

In a similar way as done for Be stars, based on the properties of our reference samples, we define selection boxes in the colourcolour diagrams to be used for our search for symbiotic stars in the Milky Way.

In the IPHAS diagram, the limited number of symbiotic stars of the D-type in the reference sample, as well as their significant overlap with the S-types (with possible differences, however, as outlined in Sect. 3.1), prevent us from defining separate boxes for the two classes. We prefer instead to use the same selection criterion, leaving to the near-IR colours the task of separating the two classes according to the original definition. Our selection box for symbiotic stars (Fig. 5, upper panel) is then defined as follows. In the $r-i$ axis, we allow the range of intrinsic colours spanned by known objects as discussed at the end of Sect. 3.1, and extend it to the right side of the graph to allow for the significant extinction that is expected when observing through the Galactic Plane.

In the vertical direction, we define a lower limit which is an inclined line, parallel to the reddening vector (Sect. 3.1), and defined by the formula $(r-\mathrm{H} \alpha) \geq 0.25 \cdot(r-i)+0.65$, which includes all known symbiotic stars in the samples of Sect. 3.1 except for the yellow symbiotic BD-213873. This limit roughly corresponds to $\mathrm{H} \alpha$ equivalent widths of $50 \AA$ (Drew et al. 2005). We decided to fix the lowest side of the selection box at this limit in order to avoid significant mixing with Be stars (see Sect. 4.1). The upper limit of the selection box for symbiotic stars is the $r-\mathrm{H} \alpha$ value for pure $\mathrm{H} \alpha$ emitters, namely $r-\mathrm{H} \alpha \sim 3.1$.

In the 2MASS diagram, the large number of objects available allows us to define separate boxes for the $\mathrm{S}$ and D-type symbiotic stars. For the S-types, we first set the lower limit for the $J-H$ colour which includes all known objects. Then the left and right limits for the $H-K_{\mathrm{S}}$ colours were chosen to run parallel to the reddening vector. They are chosen to include the vast majority of S-type systems, except for nine objects (7\% of the whole sample) that are detached and on the right side - these will, in any case, be included in the selection box for D-types. We have decided to keep the right limit for S-types (the most frequent class of symbiotics) as leftward as possible, in order to minimize mixing with T Tauri stars (even if at the expense of the D-types). The selection box for S-type symbiotic stars is indicated by the solid line in the lower panel of Fig. 5. For the D-types, we use the same procedure, with the further assumption of setting the same lower limit for the $J-H$ colour as for the S-types, and the $H-K_{\mathrm{S}}$ left limit so as to have contiguous boxes with no gap or overlap. The selection box for the D-type symbiotics is indicated by a dashed line in Fig. 5.

In this way, the three yellow symbiotics AS 201, StHA 190, and V471 Per, fall out of both boxes in the 2MASS diagram. But they are located in the very populated region of the diagram where not only main sequence stars are found, but also other $\mathrm{H} \alpha$ emitters like Be stars and cataclysmic variables. With so much mixing, a search for this kind of object becomes very difficult.

When our selection criteria are applied to the list of Witham et al. (2008), 337 sources fall in both the IPHAS and 2MASS selection boxes for the S-types (Fig. 6, left), and 846 fulfill the criteria to be considered D-type candidates (Fig. 6, right). The complete list of all these candidates is reported in Table 1, which is only available electronically. In the 2MASS diagram, a good number of objects fall in the lower right part of the selection boxes for S-type symbiotic stars, while most of the objects in the D-type box fall in its left-bottom side. These are the regions where $\mathrm{T}$ Tauri stars are also expected to be found, indicating that significant mixing of the two classes of objects is likely to be present in the candidate list (Table 1).

Our selection method recovers the three known symbiotic stars included in the list of Witham et al. (2008), as well as two suspected ones. Out of the 29 objects listed by Witham et al. (2008) as known young stellar objects, 13 are included in the list of D-type candidate symbiotics, and 3 in the list of S-type candidates (note that none of them is classified as a Be star). This confirms that the mixing with young stellar objects is more severe for the D-types. Among the 9 known or newly discovered PNe included in the list of Witham et al. (2008), only one enters the list of D-type candidates. Its nature will be discussed in Viironen et al. (2008). 

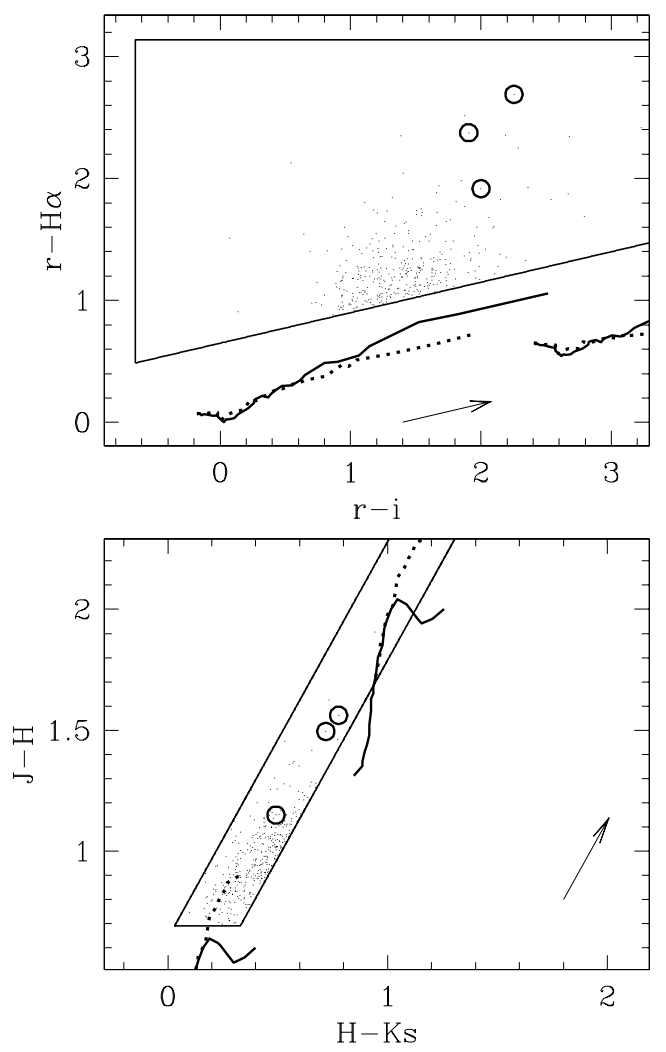
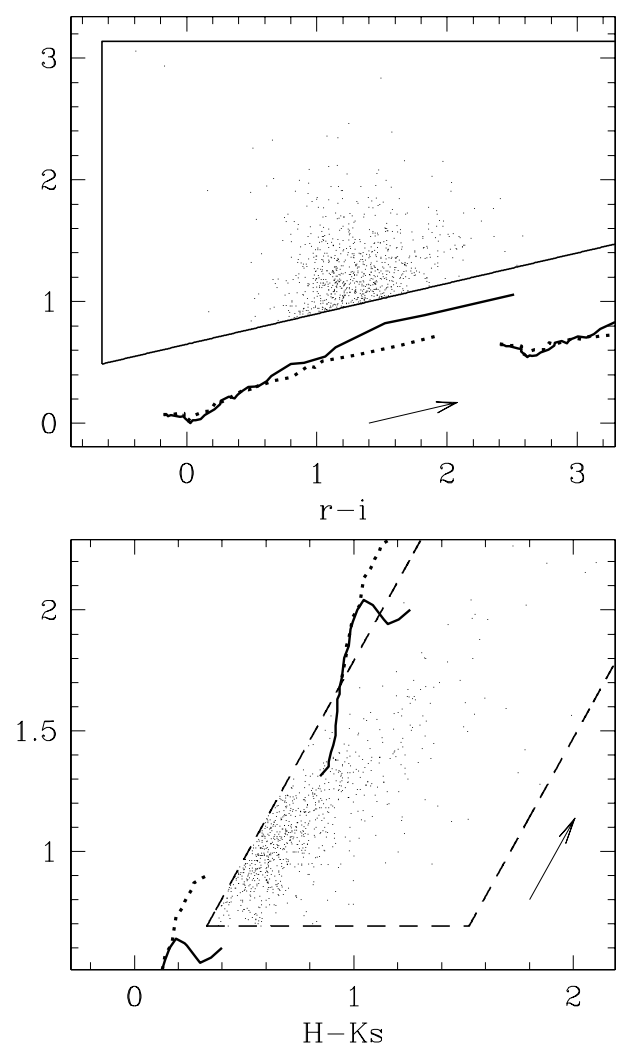

Fig. 6. Left: the 337 objects with IPHAS and 2MASS colours fulfilling our selection criteria for S-type symbiotic stars. The locations of the three new symbiotic stars discovered by IPHAS, for which we present spectroscopic confirmation in Sect. 5, are indicated by the circles. Right: the 846 candidate D-type symbiotic stars.
Note that if the lowest edge of the IPHAS selection box is lowered so as to include all known symbiotic stars in our samples, then another 152 candidates of S-type, plus 330 of D-type, would be added. At the same time, the confusion with other classes of object would also increase. In this respect, we stress that our aim is not to define absolute limits for the colours of symbiotic stars, but only to provide a practical way of selecting new candidates from the IPHAS survey, guided by the best reference samples that we were able to build from the literature, IPHAS itself, and from additional observations obtained in backup time during our observational campaign. As demonstrated below, the proposed selection method seems indeed to be promising.

\subsection{Clustering as an additional criterion to separate young stars from symbiotics}

Observations targeting a narrow band of the Galactic Plane must include a large number of star-forming regions, young clusters and associations. There, numerous objects, especially Be and $\mathrm{T}$ Tauri stars, are $\mathrm{H} \alpha$ emitters. We have shown that while Be stars can be separated from symbiotic stars because of their smaller $r-\mathrm{H} \alpha$ and $J-H$ colours, T Tauri stars overlap with symbiotics in both the IPHAS and 2MASS diagrams. Therefore, they must be regarded as the most serious "contaminants" in our search for symbiotic stars using IPHAS and 2MASS data.

Our strategy for tackling the problem is to consider the spatial distribution of the $\mathrm{H} \alpha$ emitters selected by IPHAS. T Tauri stars are expected mainly to be concentrated in young clusters. On the other hand, symbiotic stars, which belong to an older Galactic stellar population (bulge/thick-disc, Munari \& Renzini 1992), should be more isolated $\mathrm{H} \alpha$ sources (except for projection effects through the Galactic Plane).

A first step in exploring this issue was taken by measuring the degree of clustering of the objects in the list of

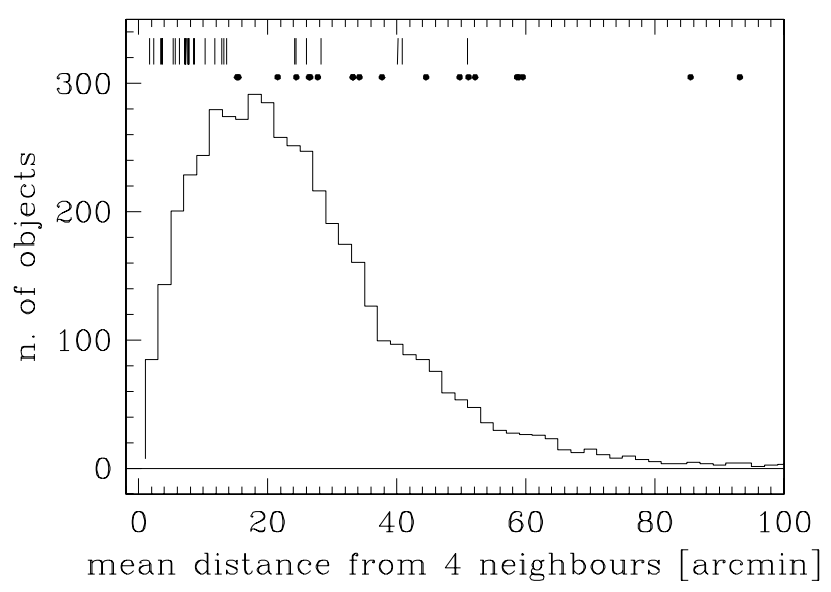

Fig. 7. Histogram of the mean distance to the four most nearby objects for each of the $4853 \mathrm{H} \alpha$ emitters by Witham et al. (2008). 29 young stellar objects are indicated by the short lines at the top of the diagram, while $20 \mathrm{PNe}$ and symbiotic stars are indicated by the dots.

Witham et al. (2008). For each of the $4853 \mathrm{H} \alpha$ emitters, the mean distance to the $n$ most nearby objects (with $n$ from 1 to 12) was computed. We show in Fig. 7 the distribution of the distances for $n=4$, that we find to be a good compromise between having a sufficient number of neighbours to detect the existence of a group, while avoiding the limited statistics that the requirement of a large number of neighbours might suffer from, given the size of the global sample.

The 29 objects listed by Witham et al. (2008) as young stellar objects, as well as 20 known, suspected or confirmed new PNe (Viironen et al. 2008) and symbiotic stars (to be presented in Paper II) were then considered. They confirm the expected trend: young stars (the vertical short lines at the top of Fig. 7) tend to occur with smaller angular separations than do PNe and 

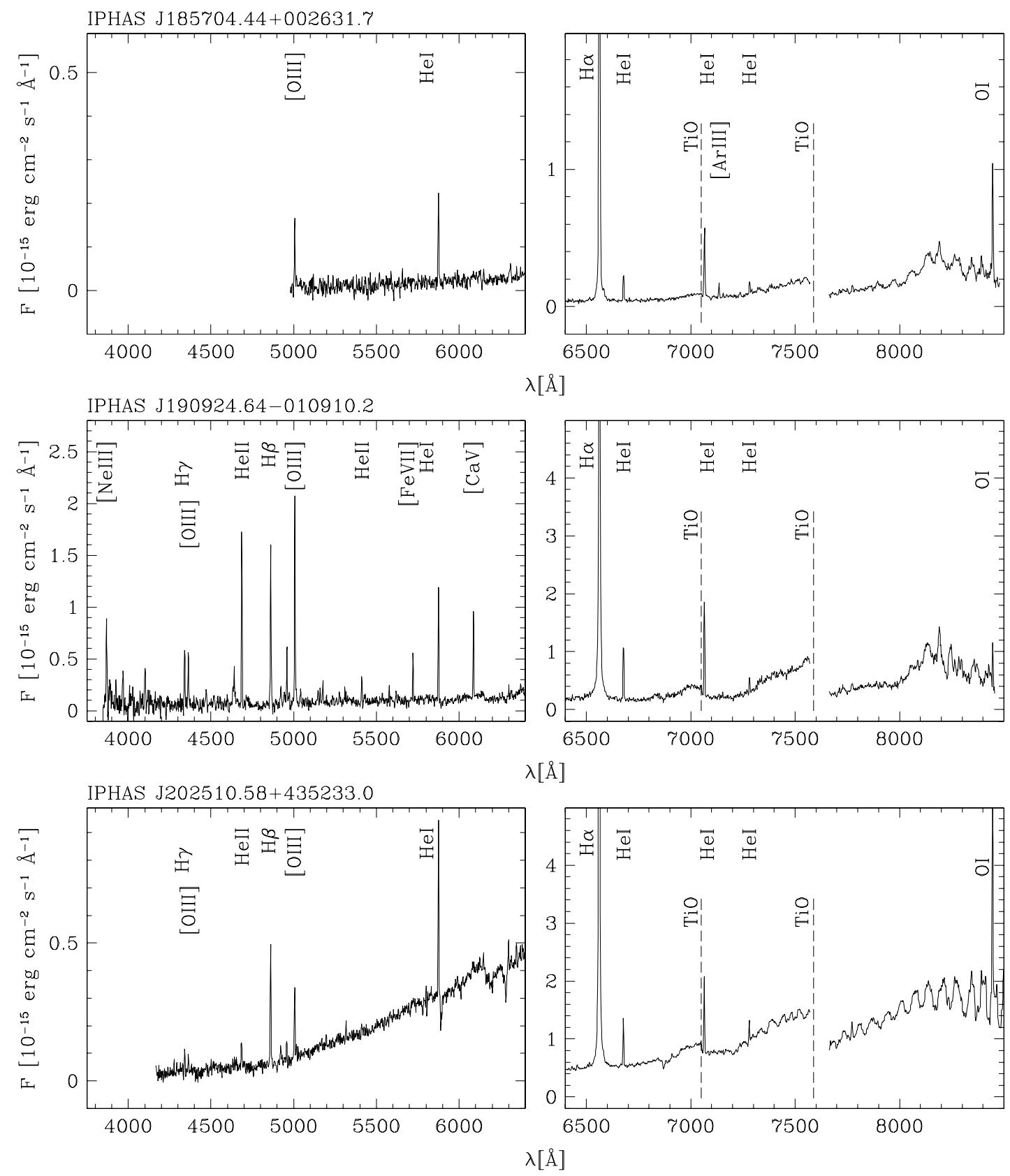

Fig. 8. Spectra of the first three new symbiotic stars discovered by IPHAS. The region around $7600 \AA$ with the strong oxygen atmospheric absorption band is not plotted. The main low and high excitation emission lines and the heads of the TiO bands, which demonstrate the symbiotic nature of the objects, are labeled. The oscillations in the very red part of the spectra are due to fringing in the detector.

symbiotic stars (the filled circles). Therefore, this additional parameter seems to be a useful one to distinguish, at least in a statistical sense, young stellar objects from stars belonging to older Galactic populations like symbiotic binaries. We expect that the significance of this parameter will improve further as the sample of $\mathrm{H} \alpha$ emitters detected by IPHAS grows in number; this would allow a better definition of clustering in the area observed by IPHAS. For the time being, we add the mean distance to the four nearest neighbours as a further datum in the list of candidate symbiotic stars in Table 1; the weight to be given to this parameter is left to the discretion of the user. In any case, we note that about $50 \%$ of both the S-type and D-type candidates in Table 1 have a mean distance to their four nearest neighbours that exceeds 15 arcmin: these candidates are our preferred initial targets for spectroscopic follow-up.

\section{Spectroscopic confirmation: the first symbiotic stars discovered by IPHAS}

As an illustration of the potential of IPHAS and the selection method proposed in the previous sections, we present here the spectra of the first three new symbiotic stars discovered by IPHAS. Data for more stars and a deeper analysis of the individual objects will be presented in Paper II.

In the following, we use both the standard nomenclature for IPHAS point sources ("IPHAS J" followed by the J2000 coordinates, see Drew et al. 2005), as well as the name "IPHAS-Sy $n n$ " to indicate the $n n$th symbiotic star discovered by the survey.

The INT spectra are shown in Fig. 8, and the location of the objects in the IPHAS and 2MASS diagrams is indicated by open circles in Fig. 6. The three objects have large $r-\mathrm{H} \alpha$ colours 
and fall in the selection box for S-type symbiotics. In the latter, two of them have colours characteristic of strongly reddened red giants, as confirmed by the INT spectra.

\subsection{IPHAS J185704.44+002631.7 (IPHAS-Sy 1)}

The IPHAS and 2MASS magnitudes of IPHAS-Sy 1 are: $r=$ $18.28, i=16.03, \mathrm{H} \alpha=15.59, J=10.42, H=8.86$, and $K_{\mathrm{S}}=$ 8.09. No objects are listed in the SIMBAD database, within a radius of 2 arcmin about the source coordinates, and the nearest known radio HII detection is over 30 arcmin away. There is no evidence to link this object to a known star-forming region.

The $\mathrm{H} \alpha$ equivalent width is $800 \AA$, which corresponds to $r-\mathrm{H} \alpha=2.69$. This is the faintest objects of the three in the optical (but not in the near-IR), and the one with the highest $J-H$ colour. This can be mainly ascribed to reddening, as shown by the steep decrease of the flux below $5000 \AA$ (no emission is detected blueward of the [OIII]5007 $\AA$ line) and the large $r-K_{\mathrm{S}}$ colour amounting to $10.2 \mathrm{mag}$. The symbiotic nature of IPHASSy 1 is indicated by the simultaneous presence of high excitation emission lines of [OIII] and [ArIII], and the TiO absorption bands of a M-type star (Fig. 8, upper panel).

\subsection{IPHAS J190924.64-010910.2 (IPHAS-Sy 2)}

IPHAS-Sy 2 has the following magnitudes: $r=17.16, i=15.26$, $\mathrm{H} \alpha=14.79, J=11.44, H=10.29$, and $K_{\mathrm{S}}=9.80$. Its $\mathrm{H} \alpha$ equivalent width is $650 \AA(r-\mathrm{H} \alpha=2.38)$.

In SIMBAD, it is indicated as a possible PN (K 4-17), but the spectrum described by Stenholm \& Acker (1987) does not allow its real nature as a symbiotic star to be identified. This is instead revealed by our INT spectroscopy, which captures a rich set of high excitation emission lines ([NeIII], [OIII], HeII, and even [FeVII]), accompanied by the red continuum of an M4 giant (Fig. 8, middle panel). No star formation regions are catalogued in the vicinity of IPHAS-Sy 2.

\subsection{IPHAS J202510.58+435233.0 (IPHAS-Sy 3)}

Its magnitudes are: $r=15.34, i=13.34, \mathrm{H} \alpha=13.42, J=9.94$, $H=8.45$, and $K_{\mathrm{S}}=7.73$. The $\mathrm{H} \alpha$ equivalent width is $250 \AA$ $(r-\mathrm{H} \alpha=1.92)$.

IPHAS-Sy 3 is listed in SIMBAD as a misclassified planetary nebula (PN K 3-59), but the spectrum of Sabbadin et al. (1987) only reveals weak $\mathrm{H} \alpha$ line emission superimposed on a very red continuum. Our observations show the composite spectrum defining a symbiotic star, with the simultaneous presence of the continuum of an early $M$ star and high excitation emission lines.

In projection, the star is located in the Cygnus $\mathrm{X}$ complex, a little over 3 arcmin from the HII region DWB 137 (Dickel et al. 1969). The vicinity of young stars might cast some doubt on the nature of IPHAS-Sy 3 as a symbiotic star. Even if it shows some of the typical spectral signatures of these interacting binaries, such as the TiO bands together with highly ionized $\mathrm{HeII}$ and $[\mathrm{OIII}]$ in emission, some extreme examples of T Tauri stars also present them (see e.g. Beristain et al. 2001).

However, there are several arguments pointing toward IPHAS-Sy 3 as most likely being a symbiotic star. First, its spectrum lacks evidence of other features typical of T Tauri stars: e.g. the strongest resonance Li doublet at $6708 \AA$ in absorption; any of FeII or $\mathrm{NaI}$ lines in emission; apparent broadening of the HeI emission line profiles. Second, T Tauri stars, being closer to the main sequence (MS), have radii just 2-3 times larger than their MS descendants, whereas symbiotic stars must contain a red giant with radii two orders of magnitudes larger than MS values. Playing devil's advocate, we can see if a typical T Tauri star radius works for IPHAS Sy 3 if placed at a plausible distance to be in a young Cygnus- $X$ cluster. The distance to the young cluster containing the Herbig Be star V1685 Cyg, some 3 degrees away on the sky, is given as $980 \mathrm{pc}$ (Davies et al. 2001), with the other nearby HII regions in Cygnus $\mathrm{X}$ being mostly at 1-2 kpc (Straizys et al. 1993). For a distance of $\sim 1 \mathrm{kpc}$, an M0 spectral type (compatible with the strength of the TiO and VO absorption bands), a reddening $E(B-V) \sim 2-2.5$ (estimated from HI and $\mathrm{HeI}$ emission line ratios, the $J-K_{\mathrm{S}}$ colour, and a fit of the optical and near-IR spectral energy distribution), we apply the Barnes-Evans relation (e.g. Beuermann et al. 1999) to derive a radius $\sim 30 R_{\odot}$. This is too large for a T Tauri star, but consistent with the presence of a red giant. The estimated radius increases if the distance is larger than $1 \mathrm{kpc}$.

\section{Summary and perspectives}

Searching for a relatively rare and old family of stars in a narrow band in the Galactic plane, where young stellar objects dominate the population of $\mathrm{H} \alpha$ emitters, might seem a hard challenge. Our motivation to embark in such an enterprise is the poor knowledge of the total population of symbiotic stars in the Galaxy: the IPHAS survey gives us the opportunity to tackle this problem by performing, for the first time, a complete search of these objects in a magnitude limited volume.

As symbiotic systems contain a cool giant star, the near-IR data from the 2MASS survey have been added to the IPHAS photometry. Then, a discussion of the location of the different classes of $\mathrm{H} \alpha$ emitters in the combined IPHAS and 2MASS colour-colour diagrams has been presented. This allows us to define selection criteria for symbiotic stars which separate them from the vast majority of normal and $\mathrm{H} \alpha$ emitting stars.

The only exception are T Tauri stars. They overlap with symbiotic stars in the IPHAS diagram. In the 2MASS one, they form a sequence very close to (and partially overlapping with) the main subclass of symbiotic stars (S-types), and fully overlap with the other, less frequent group of symbiotics (the D-types). In fact, even when a spectrum is available (see Sect. 5.3), it is sometimes difficult to distinguish a symbiotic star from those $\mathrm{T}$ Tauri stars which display high excitation emission lines. As an additional criterion to separate symbiotic from T Tauri candidates in our survey, we have considered the spatial distribution of the $\mathrm{H} \alpha$ emitters detected by IPHAS. T Tauri stars are expected to be found in groups in star forming regions. In contrast, symbiotic stars should be more isolated objects. With this aim, we have introduced a "clustering" parameter which is the mean distance to the four most nearby $\mathrm{H} \alpha$ emitters in the IPHAS list that we have considered (Witham et al. 2008).

We expect that all these criteria for the search for symbiotic stars within IPHAS will improve as soon as additional information is gathered from the survey itself. A better fix on the number of $\mathrm{H} \alpha$ emission line stars above specified, more exacting equivalent width thresholds, might improve our estimates of the clustering parameter - or further follow-up spectroscopy of the selected candidates can improve our understanding of the IPHAS and near-infrared colour-colour planes.

With this second aim in mind, we have started an intensive spectroscopic campaign. With it, we hope that the number of symbiotic stars in the IPHAS area, so far limited to only 11 objects listed in the catalogue of Belczyński et al. (2000), will 
significantly increase. The confirmation in this paper of three new systems out of a small sample of candidates observed in the first nights of the spectroscopic follow-up, seems to be a good start in this direction.

Acknowledgements. We are grateful to many of our collaborators in the IPHAS project, for continuous discussion about the properties of the variety of objects that are involved in the analysis of the survey data. R.L.M.C., E.R.R.F., A.M., and K.V. acknowledge funding from the Spanish AYA2002-0883 grant, and JM funding from Polish KBN 1PO3D 01727 grant.

\section{References}

Acker, A., \& Stenholm, B. 1990, A\&AS, 86, 219

Allen, D. A. 1984, PASA 5, 369

Allen, D. A., \& Glass, I. S. 1974, MNRAS, 167, 337

Belczyński, K., Mikolajewska, J., Munari, U., Ivison, R. J., \& Friedjung, M. 2000, A\&AS, 146, 407

Beristain, G., Edwards, S., \& Kwan, J. 2001, ApJ, 551, 1037

Beuermann, K., Baraffe, I., \& Hauschildt, P. 1999, A\&A, 348, 524

Corradi, R. L. M. 1995, MNRAS, 276, 521

Corradi, R. L. M., 2003, in Symbiotic stars probing stellar evolution, ASP Conf. Ser., 303, 393

Corradi, R. L. M., Mikolajewska, J., \& Mahoney, T. J., 2003, Symbiotic stars probing stellar evolution, ASP Conf. Ser., 303

Dahm, S. E., \& Simon, T. 2005 AJ, 129, 829

Davies, R. I., Tecza, M., Looney, L. W., et al. 2001, ApJ, 552,692

Dickel, H. R., Wendker, H., \& Bieritz, J. H. 1969, A\&A, 1, 270

Drew, J., Greimel, R., Irwin, M. J., et al. 2005, MNRAS, 362, 753

González-Solares, E. A., Walton, N., Greimel, R., et al. 2008, MNRAS, submitted
Hachisu, I., Kato, M., \& Nomoto, K., 1999, ApJ, 519, 314

Hawley, S. L., Gizis, J. E., \& Reid, I. N. 1996, AJ, 112, 2799

Howart, I. D. 1983, MNRAS, 203, 301

Jordan, S., Schmutz, W., Wolff, B., Werner K., \& Mürset U., 1996, A\&A, 312, 897

Kenyon, S. J. 1986, The Symbiotic Stars (Cambridge University Press)

Kenyon, S. J., Livio, M., Mikolajewska, J., \& Tout, C. A. 1993, ApJ, 407, L81

Magrini, L., Corradi, R. L. M., \& Munari, U., 2003, in Symbiotic stars probing stellar evolution, ASP Conf. Ser., 303, 539

Munari U., 1997, in Physical processes in Symbiotic binaries and related systems, ed. J. Mikolajewska, Copernicus Found. for Polish Astron., Warsaw, 37

Munari, U., \& Renzini, A. 1992, AJ, 397, 87

Munari, U., Yudin, B. F., Taranova, O. G., et al. 1992, A\&AS, 93, 383

Phillips, J. P. 2007, MNRAS, 376, 1120

Ramos-Larios, G., \& Phillips, J. P. 2005, MNRAS, 357, 732

Rodríguez-Flores, E. R. 2006, DEA Thesis, University of La Laguna, Tenerife, Spain

Sabbadin, F., Falomo, R., \& Ortolani, S. 1987 A\&ASS, 67, 541

Santander-García, M., Corradi, R. L. M., \& Mampaso, A. 2007, in Asymmetrical Planetary Nebulae IV, ed. R. L. M. Corradi, A. Manchado, \& N. Soker, in preparation

Schmeja, S., \& Kimeswenger, S. 2001, A\&A, 377, L18

Stenholm, B., \& Acker, A. 1987, A\&ASS, 68, 51

Straizys, V., Kazlauskas, A., Vansevicius, V., \& Cernis, K. 1993, Baltic Astron., 2,171

Tomov, T. 2003, in Symbiotic stars probing stellar evolution, ASP Conf. Ser., 303, 376

Viironen, K., et al. 2008, A\&A, in preparation

Vink, J. S., Drew, J. E., Greimel, R., et al. 2007, MNRAS, in preparation

Witham, A. R., Knigge, C., Gänsicke, B. T., et al. 2006, MNRAS, 369, 581

Witham, A. R., Knigge, C., Drew, J. E., et al. 2008, MNRAS, submitted

Zhang, P., Chen, P. S., \& Yang, H. T. 2005, New Astron., 10, 325 
R. L. M. Corradi et al.: IPHAS and the symbiotic stars. I., Online Material $p 1$

Table 1. 1183 candidate symbiotic stars extracted from the list of Witham et al. (2008) using the photometric constraints defined in Sect 4.3. Columns contain: the coordinates, the IPHAS and 2MASS magnitudes, and the mean distance $d_{4}$ (in arcmin) from four neighbours (see Sect. 4.4). Part A contains the candidate S-type symbiotics, and Part B the candidate D-types.

\begin{tabular}{|c|c|c|c|c|c|c|c|c|}
\hline \multirow{2}{*}{\multicolumn{2}{|c|}{$\begin{array}{c}\text { RA (J2000.0) Dec } \\
\text { Part A: S-type candidates }\end{array}$}} & \multirow[t]{2}{*}{$r$} & \multirow[t]{2}{*}{$i$} & \multirow[t]{2}{*}{$\overline{\mathrm{H} \alpha}$} & \multirow[t]{2}{*}{$\bar{J}$} & \multirow[t]{2}{*}{$\bar{H}$} & \multirow[t]{2}{*}{$\overline{K_{\mathrm{s}}}$} & \multirow[t]{2}{*}{$d_{4}$} \\
\hline & & & & & & & & \\
\hline 000740.09 & +654010.6 & 17.07 & 15.80 & 15.56 & 13.98 & 13.14 & 12.84 & 6.7 \\
\hline 000834.71 & +652832.8 & 18.54 & 17.06 & 17.12 & & .87 & 3.55 & 10.8 \\
\hline 001033.27 & +584021.1 & 16.00 & 14.40 & 14.51 & 12.34 & 11.45 & 11.00 & 4.7 \\
\hline 001036.41 & +585005.0 & 14.66 & 13.70 & 13.06 & 11.89 & 11.09 & 10.74 & 7.2 \\
\hline 001301.79 & +653445.7 & 18.89 & 17.23 & 17.00 & 14.72 & 13.80 & 13.38 & 15.2 \\
\hline 001428.55 & +654934.5 & 19.42 & 17.32 & 17.82 & 15.11 & 4.19 & 13.78 & 5.8 \\
\hline 001520.95 & +654941.1 & 18.59 & 16.61 & 17.14 & 14.28 & 13.36 & 2.98 & 6.1 \\
\hline 001522.04 & +654530.4 & 16.68 & 15.49 & 15.13 & 13.47 & 12.54 & 12.09 & 6.2 \\
\hline 001909.80 & +623401.5 & 19.16 & 17.82 & 17.97 & 16.10 & 15.34 & 15.17 & 11.6 \\
\hline 002106.03 & +621049.1 & 19.37 & 17.93 & 18.33 & & & 14.55 & 17.9 \\
\hline 2205.18 & +623611.1 & 18.57 & 17.06 & 17.54 & 14.45 & .39 & 13.08 & 14.1 \\
\hline 2718.55 & +644431.3 & & & 18.21 & & .23 & .72 & 22.6 \\
\hline 003031.28 & +653202.1 & 18.51 & 16.84 & 16.79 & 14.71 & 13.65 & 13.16 & 4.6 \\
\hline 003047.88 & +652122.1 & 18.65 & 17.00 & 17.58 & 14.32 & 13.20 & 12.61 & 6.9 \\
\hline 3152.06 & +652808.3 & 18.38 & 16.94 & 17.24 & 14.95 & .82 & 13.34 & 8.3 \\
\hline 24.83 & +655930.9 & & & 16.57 & & 36 & 82 & 25.3 \\
\hline 601.76 & +623235.6 & 18.56 & 17.1 & 17.39 & & 52 & 12.91 & 33.7 \\
\hline 003927.23 & +601900.3 & 18.67 & 17.55 & 17.62 & 15.89 & 14.99 & 14.66 & 33.4 \\
\hline 004110.36 & +621054.0 & 17.72 & 15.59 & 16.44 & 13.38 & 12.60 & 12.25 & 20.2 \\
\hline 4153.24 & +614942.8 & .15 & 16. & 16.82 & 3.89 & .03 & 12.64 & 9.3 \\
\hline 004212.35 & +61544 & & & 15.92 & & 07 & 1.71 & 7.7 \\
\hline 004252.37 & +611039.3 & 18.24 & & 16.90 & 04 & 12.99 & 12.58 & 6.1 \\
\hline 012305.28 & +614200.8 & 18.24 & & 16.96 & 14.80 & 3.96 & 13.68 & 14.6 \\
\hline 012341.80 & +615001.0 & 17.24 & 15. & 16.26 & 13.77 & 12.75 & 12.26 & 15.1 \\
\hline 12701.54 & +623 & & & 06 & & 16 & .83 & 19.2 \\
\hline 013837.90 & +645747.3 & 17.97 & 16.59 & 16.75 & 61 & 57 & .06 & 28.4 \\
\hline 022307.88 & +565807.2 & & 17.43 & 17.42 & & 1.73 & 14.49 & 34.0 \\
\hline 022759.30 & +585147.9 & 19.20 & 18.00 & 17.48 & 15.93 & 14.85 & 14.30 & 28.3 \\
\hline 022816.91 & +620950.8 & 18.67 & & 17.49 & .38 & .35 & 13.86 & 14.1 \\
\hline 022901.93 & +620725.2 & & 17.7 & 17.42 & & 92 & 14.64 & 17.3 \\
\hline 023142.96 & +610453.0 & 19.30 & & 18.29 & & 15 & 89 & 10.3 \\
\hline 023251.54 & +614346.8 & & & 17.26 & & & 13.74 & 7.6 \\
\hline 3253.77 & +621528.9 & 17.70 & 16.7 & 16.71 & 15.10 & 14.25 & 13.96 & 20.6 \\
\hline 023657.74 & +612857.1 & 18.26 & 16. & 17.24 & 04 & & 3.53 & 10.5 \\
\hline 023701.50 & +612823.6 & 0 & & 5.67 & & .67 & 17 & 10.8 \\
\hline 023749.30 & +605107.3 & 19.24 & 17.99 & 18.08 & 99 & 83 & .26 & 14.9 \\
\hline 023814.81 & +615214.6 & 18.23 & 17.13 & 17.06 & 15.21 & 14.38 & 14.11 & 11.3 \\
\hline 024148.15 & +572337.1 & 19.03 & 17.85 & 17.87 & & .34 & 14.93 & 15.3 \\
\hline 025045.30 & +602809.4 & 18.04 & 16.80 & 17.01 & 14.76 & .74 & 13.27 & 10.3 \\
\hline 025211.62 & +602951.5 & & 17. & 17.38 & & & 01 & 8.2 \\
\hline 025248.11 & +601609.6 & 18.71 & 17.54 & 17.20 & 15 & 14.25 & 13.78 & 7.5 \\
\hline 025526.52 & +572050.6 & 18.07 & & 17.09 & & .93 & 13.58 & 25.5 \\
\hline 025719.80 & +602141.3 & 19.47 & 18.20 & 18.21 & 16.37 & .34 & 15.01 & 10.7 \\
\hline 025749.09 & +604617.9 & 18.78 & 17.62 & 17.76 & 15.61 & 14.72 & 14.38 & 6.7 \\
\hline 025820.50 & +603558.4 & 18.95 & 17.72 & 17.69 & 15.67 & 14.61 & 14.07 & 4.8 \\
\hline 025845.13 & +602942.1 & 19.12 & 17.80 & 17.96 & 15.49 & 14.66 & 14.29 & 4.4 \\
\hline 025917.69 & +603411.0 & 19.43 & 17.90 & 18.16 & 15.53 & 14.46 & 13.98 & 2.8 \\
\hline 025931.03 & +604025.5 & 18.75 & 17.54 & 17.36 & 15.25 & 14.15 & 13.63 & 5.0 \\
\hline 030007.58 & +602536.8 & 19.08 & 17.91 & 17.84 & 15.70 & 14.56 & 14.12 & 4.4 \\
\hline 030043.98 & +603059.0 & 18.76 & 17.55 & 17.53 & 15.45 & 14.43 & 13.97 & 3.8 \\
\hline 031147.12 & +563430.2 & 18.76 & 17.29 & 17.42 & 14.94 & & 13.26 & 29.1 \\
\hline 031704.34 & +601500.0 & 16.65 & 15.33 & 15.21 & 13.33 & 12.35 & 11.91 & 11.8 \\
\hline 031937.54 & +591114.4 & 18.82 & 17.43 & 17.64 & 15.31 & 14.36 & 14.05 & 25.4 \\
\hline 032020.46 & +601632.9 & 18.59 & 17.12 & 17.17 & 14.89 & 14.07 & 13.69 & 4.1 \\
\hline 032046.82 & +602033.0 & 17.96 & 16.58 & 16.69 & 14.63 & 13.62 & 13.13 & 4.2 \\
\hline 032048.19 & +602019.0 & 16.70 & 15.59 & 15.51 & 13.63 & 12.74 & 12.38 & 4.1 \\
\hline 032542.41 & +585730.0 & 18.68 & 17.21 & 17.43 & 15.26 & 14.39 & 14.05 & 14.5 \\
\hline 032647.39 & +585538.3 & 17.95 & 16.51 & 16.60 & 14.05 & 12.89 & 12.31 & 9.1 \\
\hline
\end{tabular}


R. L. M. Corradi et al.: IPHAS and the symbiotic stars. I., Online Material $p 2$

Table 1. continued.

\begin{tabular}{|c|c|c|c|c|c|c|c|c|}
\hline \multicolumn{2}{|c|}{ RA (J2000.0) Dec } & $r$ & $i$ & $\mathrm{H} \alpha$ & $J$ & $\bar{H}$ & $\overline{K_{\mathrm{s}}}$ & $d_{4}$ \\
\hline 033232.97 & +600825.2 & 18.93 & 17.31 & 17.77 & 14.84 & 13.80 & 13.32 & 20.7 \\
\hline 034335.34 & +530940.6 & 18.25 & 16.72 & 17.13 & 14.37 & 13.29 & 12.86 & 37.9 \\
\hline 034422.86 & +540729.0 & 18.64 & 17.32 & 17.19 & 15.09 & 14.06 & 13.76 & 30.6 \\
\hline 034423.39 & +564636.3 & 18.51 & 17.15 & 17.41 & 15.27 & 14.41 & 14.19 & 32.5 \\
\hline 035922.23 & +515415.1 & 17.09 & 16.09 & 16.12 & 14.35 & 13.54 & 13.37 & 13.5 \\
\hline 040232.14 & +513958.1 & 18.20 & 16.78 & 17.14 & 14.75 & 13.68 & 13.24 & 25.5 \\
\hline 040732.70 & +573831.4 & 16.74 & 15.73 & 15.74 & 14.05 & 13.23 & 13.02 & 20.4 \\
\hline 041642.06 & +551444.2 & 18.59 & 17.90 & 17.18 & 16.51 & 15.77 & 15.48 & 23.2 \\
\hline 042334.84 & +501822.5 & 18.39 & 17.22 & 17.42 & 15.51 & 14.34 & 13.74 & 41.5 \\
\hline 042604.19 & +453422.7 & 19.26 & 17.64 & 17.71 & 15.22 & 13.95 & 13.28 & 29.4 \\
\hline 042907.66 & +452647.1 & 18.42 & 17.53 & 17.28 & 15.67 & 14.96 & 14.68 & 9.2 \\
\hline 3100.52 & +445559.6 & 19.25 & 18.02 & 18.13 & 16.09 & 14.84 & 14.46 & 27.3 \\
\hline 043629.20 & +451750.4 & 18.08 & 16.86 & 17.04 & 15.18 & 14.31 & 14.04 & 18.7 \\
\hline 044707.00 & +434339.0 & 18.30 & 17.07 & 17.24 & 15.44 & 14.62 & 14.36 & 24.7 \\
\hline 045630.88 & +435748.9 & 18.84 & 17.40 & 17.77 & 15.31 & 14.37 & 13.96 & 40.2 \\
\hline 5638.58 & +450225.9 & 15.08 & 14.30 & 14.12 & 12.90 & 12.09 & 11.71 & 36.5 \\
\hline 5.64 & +434143.5 & 15.91 & 14.90 & 14.73 & 2.16 & 1.46 & 1.15 & 34.1 \\
\hline 051105.67 & +423621.3 & 18.18 & 16.87 & 17.13 & 15.39 & 14.67 & 14.49 & 35.9 \\
\hline 051201.82 & +384334.0 & 18.97 & 18.03 & 17.93 & 15.70 & 14.67 & 14.24 & 12.9 \\
\hline 051827.42 & +300849.7 & 13.76 & 12.40 & 12.70 & 10.59 & 9.89 & 9.64 & 65.0 \\
\hline 53.15 & 845.8 & 19.06 & 17.78 & 18.05 & 49 & 14.47 & 4.10 & 51.8 \\
\hline 052241.49 & +332050.1 & 18.44 & 17.39 & 17.42 & 15.54 & 14.38 & 13.91 & 7.4 \\
\hline 052245.78 & +332816.4 & 18.30 & 17.06 & 17.19 & 15.05 & 14.04 & 13.54 & 3.5 \\
\hline 052300.06 & +333039.0 & 17.07 & 16.13 & 16.14 & 14.34 & 13.42 & 12.97 & 3.7 \\
\hline 052544.57 & +345017.9 & 17.73 & 16.63 & 16.76 & 14.71 & 13.72 & 13.23 & 10.3 \\
\hline 05263 & +345 & 17.94 & 16.97 & 16.86 & 15.26 & .21 & 13.74 & 7.4 \\
\hline 052652.58 & +403306.2 & 18.86 & 17.91 & 17.90 & 16.33 & 15.48 & 15.22 & 54.9 \\
\hline 052712.33 & +343219.8 & 18.99 & 18.05 & 17.80 & 16.12 & 15.25 & 15.01 & 5.8 \\
\hline 2716.23 & +351339.6 & 19.01 & 17.77 & 17.81 & 15.85 & 14.59 & 14.10 & 15.7 \\
\hline 052725.89 & +344241.7 & 18.15 & 17.22 & 16.99 & 15.44 & 59 & 4.31 & 4.7 \\
\hline 052743.83 & +344027.9 & 18.82 & 17.84 & 17.30 & 16.12 & 36 & 15.08 & 3.2 \\
\hline 052754.45 & +344622.2 & 17.29 & 16.55 & 16.30 & 15.23 & 49 & 14.22 & 3.8 \\
\hline 052754.76 & +344530.7 & 18.50 & 17.49 & 17.49 & 15.36 & 14.39 & 13.91 & 3.2 \\
\hline 5301 & +313 & 16.95 & 16.02 & 16.01 & 14.32 & 13.50 & 13.21 & 33.7 \\
\hline 053258.06 & +255 & 17.86 & 16.30 & 16.55 & 14.32 & 3.42 & 13.09 & 20.1 \\
\hline 053406.31 & +253822.5 & 17.29 & 16.21 & 16.11 & 10 & 13.10 & 12.59 & 9.9 \\
\hline 053426.31 & +253850.4 & 19.25 & 17.44 & 17.43 & 14.98 & 14.09 & 13.71 & 8.9 \\
\hline 3456.79 & +274736.4 & 18.00 & 16.95 & 17.00 & 15.10 & 14.24 & 13.88 & 13.2 \\
\hline 3611.81 & +321437.9 & 16.92 & 16.10 & 15.51 & 14.68 & .96 & 3.68 & 13.6 \\
\hline 053612.82 & +273410.8 & 18.09 & 16.60 & 16.96 & 10 & 2.89 & 12.29 & 12.7 \\
\hline 053641.46 & +314627.8 & 18.19 & 16.90 & 17.10 & 14.91 & 13.93 & 13.57 & 4.5 \\
\hline 053714.05 & +274857.8 & 18.89 & 17.46 & 17.64 & 15.24 & 14.12 & 13.69 & 7.0 \\
\hline 3844.29 & +261641.7 & 17.58 & 16.15 & 16.36 & 14.25 & 13.43 & 13.18 & 21.4 \\
\hline 11.84 & +264218.8 & 17.07 & 15.89 & 16.11 & 4.19 & 13.39 & 13.19 & 21.5 \\
\hline 053929.60 & +312349.5 & 18.55 & 17.21 & 17.00 & 10 & .14 & .75 & 27.7 \\
\hline 054050.08 & +352349.1 & 17.70 & 16.41 & 16.49 & 14.44 & 13.50 & 13.09 & 21.8 \\
\hline 054223.90 & +224915.4 & 19.15 & 17.87 & 17.73 & 15.97 & 14.62 & 14.02 & 31.7 \\
\hline 054333.43 & +332411.8 & 18.44 & 17.51 & 17.41 & 15.97 & 15.07 & 14.64 & 34.8 \\
\hline 054706.78 & +205913.0 & 18.68 & 17.02 & 17.35 & 14.93 & 13.83 & 13.34 & 40.0 \\
\hline 054914.66 & +332329.3 & 16.97 & 16.01 & 15.74 & 14.32 & 13.47 & 13.12 & 20.1 \\
\hline 055204.78 & +200733.8 & 17.20 & 16.29 & 16.25 & 14.84 & 14.08 & 13.84 & 65.1 \\
\hline 055837.29 & +320438.1 & 19.32 & 18.28 & 18.38 & 16.44 & 15.70 & 15.40 & 34.3 \\
\hline 055845.75 & +201012.6 & 17.79 & 16.77 & 16.76 & 14.95 & 14.11 & 13.90 & 11.4 \\
\hline 055936.44 & +203149.0 & 19.01 & 17.61 & 17.82 & 15.58 & 14.62 & 14.30 & 12.4 \\
\hline 060005.82 & +201817.8 & 19.11 & 17.59 & 18.04 & 15.51 & 14.62 & 14.34 & 9.2 \\
\hline 060134.22 & +161345.8 & 15.37 & 14.44 & 14.21 & 12.98 & 12.28 & 12.04 & 22.9 \\
\hline 060821.91 & +295255.7 & 17.80 & 16.35 & 16.39 & 14.41 & 13.37 & 12.84 & 44.2 \\
\hline 061120.03 & +132058.1 & 18.96 & 17.75 & 17.78 & 15.70 & 14.68 & 14.16 & 42.9 \\
\hline 061308.11 & +230523.1 & 17.77 & 17.64 & 16.86 & 15.45 & 14.59 & 14.34 & 19.7 \\
\hline 061317.70 & +230814.1 & 17.74 & 17.67 & 16.23 & 15.27 & 14.49 & 14.12 & 20.2 \\
\hline 061328.37 & +160455.1 & 18.11 & 16.37 & 16.46 & 13.74 & 12.93 & 12.58 & 28.5 \\
\hline
\end{tabular}


Table 1. continued.

\begin{tabular}{|c|c|c|c|c|c|c|c|c|}
\hline \multicolumn{2}{|c|}{ RA $(J 2000.0)$ Dec } & $r$ & $i$ & $\mathrm{H} \alpha$ & $\bar{J}$ & $\bar{H}$ & $\bar{K}_{\mathrm{s}}$ & $d_{4}$ \\
\hline 061419.39 & +212805.7 & 18.28 & 17.64 & 16.74 & 15.55 & 14.75 & 14.56 & 27.4 \\
\hline 061540.34 & +190005.2 & 18.49 & 17.36 & 17.39 & 15.40 & 14.45 & 14.06 & 31.0 \\
\hline 648.63 & +205633.1 & 18.84 & 17.61 & 17.48 & 15.64 & 14.58 & 14.15 & 36.0 \\
\hline 061656.26 & +23 3827.1 & 19.09 & 17.97 & 17.66 & 15.81 & 14.66 & 14.15 & 33.4 \\
\hline 062206.53 & +223436.7 & 18.61 & 16.86 & 17.40 & 14.48 & 13.37 & 12.93 & 10.2 \\
\hline 063124.23 & +121612.7 & 18.74 & 17.65 & 17.66 & 15.88 & 14.95 & 14.73 & 32.1 \\
\hline 3141.98 & +045418.2 & 16.45 & 15.58 & 15.56 & 13.89 & 13.18 & 12.90 & 4.4 \\
\hline 063154.87 & +103118.8 & 17.84 & 15.74 & 16.62 & 12.85 & 11.75 & 11.24 & 8.5 \\
\hline 063158.90 & +045811.1 & 18.16 & 17.06 & 16.89 & 15.09 & 14.19 & 13.74 & 4.7 \\
\hline 063205.29 & +045742.5 & 17.18 & 16.24 & 16.01 & 14.61 & 13.78 & 13.50 & 4.9 \\
\hline 3210.04 & +120817.8 & 19.07 & 17.83 & 18.01 & 15.78 & 14.67 & 14.16 & 37.0 \\
\hline 3216.30 & +100955.9 & 18.12 & 16.09 & 16.70 & 13.51 & 12.74 & 12.38 & 5.6 \\
\hline 063612.36 & +0343 33.7 & 18.09 & 16.88 & 16.79 & 14.84 & 13.71 & 13.15 & 38.8 \\
\hline 063659.97 & +020958.9 & 18.82 & 17.38 & 17.27 & 15.03 & 13.90 & 13.37 & 3.6 \\
\hline 53701.68 & +021046.7 & 17.41 & 16.08 & 16.43 & 14.06 & 13.12 & 12.72 & 3.6 \\
\hline 703.18 & +021 & 18.56 & 17.11 & 17.32 & 14.40 & 13.21 & 12.58 & 3.9 \\
\hline .08 & -003457.2 & 17.53 & 14.87 & 15.66 & 10.97 & 9.61 & 9.05 & 51.1 \\
\hline 183334.49 & +003422.1 & 18.01 & 16.05 & 16.82 & 13.24 & 12.09 & 11.63 & 40.0 \\
\hline 183501.83 & +014656.0 & 16.34 & 14.47 & 13.83 & 10.74 & 9.48 & 8.92 & 26.4 \\
\hline 183748.03 & -001617.2 & 16.94 & 15.51 & 15.72 & 13.25 & 12.31 & 12.04 & 42.7 \\
\hline .38 & +001 & 18.47 & 17.08 & 16.11 & 20 & 13.24 & 12.79 & 36.0 \\
\hline 183943.08 & -011116.7 & 17.79 & 16.30 & 16.71 & 13.93 & 12.99 & 12.68 & 46.5 \\
\hline 184439.63 & +050249.1 & 14.01 & 12.27 & 12.67 & 9.39 & 8.29 & 7.81 & 53.1 \\
\hline 184446.08 & +060703.5 & 14.74 & 13.34 & 12.70 & 11.04 & 9.88 & 9.41 & 44.5 \\
\hline 4628.35 & +002554.5 & 18.32 & 16.81 & 17.04 & 14.31 & 13.08 & 12.46 & 25.9 \\
\hline .03 & +032 & 18.55 & 15.32 & 16.14 & 10.07 & 8.44 & 7.71 & 54.0 \\
\hline 184839.34 & +002342.9 & 15.54 & 14.43 & 14.47 & 12.48 & 11.57 & 11.25 & 38.9 \\
\hline 185039.20 & +065916.7 & 17.93 & 15.74 & 16.57 & 12.31 & 10.86 & 10.32 & 65.7 \\
\hline .58 & +084955.1 & 16.54 & 14.25 & 14.28 & 10.32 & 9.04 & 8.52 & 85.5 \\
\hline .26 & +00 & 17.46 & 15.73 & 16.17 & 13.11 & 12.00 & 11.44 & 23.7 \\
\hline 185704.44 & +00 2631.7 & 18.28 & 16.03 & 15.59 & 10.43 & 8.86 & 8.09 & 24.4 \\
\hline 190106.87 & +045927.5 & 18.73 & 16.66 & 17.04 & 13.87 & 12.89 & 12.44 & 51.0 \\
\hline 190309.13 & +020431.8 & 18.85 & 17.53 & 17.66 & 15.23 & 14.21 & 13.74 & 74.9 \\
\hline .19 & 58.4 & 16.33 & 14.54 & 15.21 & 14.78 & 14.00 & 13.71 & 68.8 \\
\hline 1.53 & -005 & 15.88 & 13.08 & 14.19 & 8.89 & 7.75 & 7.17 & 25.9 \\
\hline 190924.64 & -010910.2 & 17.16 & 15.26 & 14.79 & 11.44 & 10.29 & 9.80 & 15.5 \\
\hline 191036.14 & +024928.2 & 15.32 & 12.79 & 13.50 & 10.08 & 8.94 & 8.51 & 37.7 \\
\hline .89 & +203 & 18.14 & 16.37 & 16.72 & 14.13 & 13.13 & 12.79 & 56.8 \\
\hline 19212 & +10 & 18.39 & 16.03 & 16.46 & 12.61 & 11.52 & 10.95 & 38.9 \\
\hline 192204.55 & +103153.7 & 19.00 & 16.32 & 16.66 & 11.89 & 9.98 & 9.04 & 35.0 \\
\hline 192336.01 & +100009.4 & 18.71 & 16.57 & 17.34 & 14.52 & 13.70 & 13.40 & 46.3 \\
\hline 192506.46 & +224514.1 & 16.24 & 15.12 & 14.99 & 13.43 & 12.51 & 12.05 & 2.4 \\
\hline 192515.05 & +224720.3 & 16.40 & 15.29 & 14.60 & 13.38 & 12.57 & 12.24 & 3.3 \\
\hline 192556.60 & +230454.0 & 17.73 & 16.08 & 16.51 & 14.31 & 13.57 & 13.38 & 18.6 \\
\hline 192629.11 & +210407.4 & 15.18 & 14.15 & 14.26 & 12.69 & 11.92 & 11.70 & 9.7 \\
\hline 192635.81 & +223924.4 & 18.00 & 16.36 & 16.68 & 13. & 12.75 & 12.21 & 8.7 \\
\hline 192712.25 & +223936.9 & 16.56 & 15.11 & 15.46 & 12.95 & 11.83 & 11.31 & 8.9 \\
\hline 192742.36 & +201825.7 & 18.43 & 16.67 & 17.18 & 14.40 & 13.64 & 13.35 & 22.5 \\
\hline 192951.43 & +215411.3 & 18.33 & 17.09 & 17.34 & 15.08 & 14.29 & 14.11 & 27.4 \\
\hline 193436.06 & +163128.9 & 16.84 & 14.65 & 14.49 & 10.71 & 9.34 & 8.77 & 52.2 \\
\hline 193501.31 & +135427.5 & 14.04 & 12.51 & 12.81 & 10.05 & 8.98 & 8.58 & 36.6 \\
\hline 193530.04 & +271147.6 & 17.35 & 15.58 & 16.05 & 14.24 & 13.49 & 13.40 & 23.6 \\
\hline 194117.05 & +220132.3 & 18.68 & 16.89 & 17.19 & 14.61 & 13.75 & 13.44 & 24.4 \\
\hline 194120.78 & +245612.8 & 14.91 & 13.30 & 13.77 & 10.39 & 9.19 & 8.73 & 29.7 \\
\hline 194223.61 & +225530.8 & 19.41 & 18.04 & 18.30 & 15.62 & 14.34 & 13.66 & 12.1 \\
\hline 194450.12 & +234722.5 & 19.05 & 17.75 & 18.02 & 15.62 & 14.42 & 14.01 & 2.9 \\
\hline 194523.91 & +235513.1 & 17.91 & 16.50 & 16.88 & 14.21 & 13.10 & 12.56 & 9.6 \\
\hline 194529.73 & +241156.5 & 19.02 & 17.71 & 17.89 & 16.00 & 14.74 & 14.28 & 13.9 \\
\hline 195910.60 & +291940.3 & 16.38 & 14.43 & 15.14 & 11.04 & 9.67 & 9.10 & 18.5 \\
\hline 200223.56 & +351656.3 & 18.77 & 17.23 & 17.50 & 14.76 & 13.75 & 13.23 & 7.0 \\
\hline 200421.29 & +354149.6 & 19.10 & 17.95 & 17.63 & 15.56 & 14.65 & 14.22 & 6.7 \\
\hline
\end{tabular}


Table 1. continued.

\begin{tabular}{|c|c|c|c|c|c|c|c|c|}
\hline \multicolumn{2}{|c|}{ RA (J2000.0) Dec } & $r$ & $i$ & $\mathrm{H} \alpha$ & $J$ & $\bar{H}$ & $\overline{K_{\mathrm{s}}}$ & $d_{4}$ \\
\hline 200427.42 & +343735.3 & 19.36 & 18.10 & 17.71 & 16.00 & 14.86 & 14.48 & 29.5 \\
\hline 200722.57 & +353832.5 & 19.21 & 17.79 & 17.57 & 15.83 & 14.69 & 14.13 & 4.7 \\
\hline 200812.32 & +35 5922.7 & 18.89 & 17.92 & 17.56 & 15.96 & 15.03 & 14.55 & 12.0 \\
\hline 200850.48 & +355410.6 & 18.67 & 17.65 & 17.74 & 16.20 & 14.98 & 14.35 & 12.3 \\
\hline 201036.18 & +300127.8 & 18.50 & 17.09 & 16.86 & 15.37 & 14.63 & 14.38 & 31.9 \\
\hline 201142.77 & +393954.2 & 15.40 & 14.34 & 14.29 & 12.42 & 11.54 & 11.15 & 32.7 \\
\hline 201156.67 & +370022.9 & 17.61 & 16.59 & 16.43 & 14.81 & 13.80 & 13.31 & 19.9 \\
\hline 201316.45 & +360630.1 & 18.54 & 17.43 & 17.54 & 15.41 & 14.33 & 13.77 & 12.5 \\
\hline 201637.58 & +401847.1 & 16.14 & 15.19 & 15.05 & 13.85 & 13.06 & 12.81 & 18.1 \\
\hline 201703.42 & +404133.9 & 17.55 & 16.13 & 16.31 & 14.53 & 13.63 & 13.37 & 9.2 \\
\hline 201713.67 & +403031.2 & 18.71 & 17.12 & 17.59 & 14.70 & 13.56 & 12.96 & 15.1 \\
\hline 201743.66 & +341907.1 & 18.90 & 17.63 & 17.80 & 15.60 & 14.47 & 13.94 & 31.0 \\
\hline 201822.11 & +391042.7 & 18.80 & 17.58 & 17.31 & 15.46 & 14.65 & 14.47 & 8.9 \\
\hline 201823.03 & +385815.9 & 18.14 & 16.96 & 16.39 & 15.07 & 14.06 & 13.70 & 8.0 \\
\hline 201845.96 & +405553.2 & 16.94 & 15.80 & 15.94 & 13.98 & 13.14 & 12.93 & 4.3 \\
\hline 58.45 & 59.9 & 18.54 & 16.96 & 17.01 & 14.80 & 13.89 & 13.48 & 14.5 \\
\hline 05.63 & +392351.3 & 18.40 & 17.22 & 17.05 & 15.11 & 4.17 & 13.78 & 5.9 \\
\hline 201913.93 & +405405.2 & 16.13 & 14.98 & 14.92 & 13.40 & 12.52 & 12.17 & 6.5 \\
\hline 201915.88 & +403542.3 & 17.74 & 16.44 & 16.64 & 14.68 & 13.68 & 13.30 & 11.4 \\
\hline 202002.64 & +401052.3 & 17.69 & 16.02 & 16.53 & 13.62 & 12.64 & 12.33 & 12.7 \\
\hline 20201 & +370 & 18.14 & 17.09 & 17.19 & 01 & .07 & .60 & 14.3 \\
\hline 202020.60 & +395243.8 & 19.50 & 17.77 & 18.01 & 15.54 & 14.54 & 14.08 & 10.7 \\
\hline 202037.46 & +395644.9 & 16.46 & 15.54 & 15.36 & 13.98 & 13.20 & 12.85 & 11.3 \\
\hline 202041.50 & +404859.1 & 16.21 & 15.29 & 15.27 & 13.87 & 13.02 & 12.71 & 12.2 \\
\hline 2047.07 & +385542.2 & 18.30 & 17.02 & 17.27 & 14.75 & 13.75 & 13.30 & 13.4 \\
\hline 20212 & +38 & 18.07 & 16.99 & 17.01 & .07 & .17 & 3.89 & 14.0 \\
\hline 202202.15 & +385050.2 & 17.20 & 16.28 & 15.92 & 14.53 & 13.49 & 12.98 & 11.6 \\
\hline 202225.44 & +384046.1 & 19.37 & 17.82 & 17.68 & 15.47 & 14.54 & 14.12 & 12.7 \\
\hline 2352.57 & +404605.8 & 17.25 & 16.32 & 16.21 & 14.88 & 14.06 & 13.75 & 15.0 \\
\hline 202426.35 & +390 & 16.69 & 15.27 & 15.60 & 12.95 & .91 & 1.38 & 3.6 \\
\hline 202445.41 & +390020.9 & 17.52 & 16.00 & 16.32 & 13.85 & 12.95 & 12.55 & 4.5 \\
\hline 202446.60 & +422142.2 & 18.04 & 16.86 & 16.86 & 14.79 & 13.90 & 13.47 & 6.2 \\
\hline 202510.58 & +435233.0 & 15.34 & 13.34 & 13.42 & 9.94 & 8.45 & 7.73 & 26.6 \\
\hline 202515.11 & +394828.1 & 16.55 & 15.48 & 15.28 & 13.67 & 12.73 & 12.28 & 28.2 \\
\hline 202717.23 & +390 & 17.00 & 15.93 & 15.59 & 13.81 & 12.85 & 2.56 & 14.2 \\
\hline 202909.03 & +393140.8 & 19.46 & 17.94 & 18.19 & 28 & 14.23 & 13.93 & 10.4 \\
\hline 202957.91 & +391913.5 & 18.62 & 17.20 & 17.54 & 15.06 & 14.21 & 13.96 & 18.8 \\
\hline 203052.48 & +401816.2 & 16.63 & 15.55 & 15.36 & 13.78 & 12.91 & 12.64 & 12.8 \\
\hline 203149.04 & +401 & 17.50 & 16.45 & 16.22 & 15.30 & 14.33 & 3.90 & 15.0 \\
\hline 203259.66 & +450402.6 & 17.93 & 16.91 & 16.86 & 94 & 13.94 & 3.47 & 19.6 \\
\hline 203545.40 & +400332.6 & 19.46 & 17.50 & 17.86 & 93 & 13.96 & 13.51 & 40.8 \\
\hline 203656.75 & +421519.1 & 17.73 & 16.23 & 16.60 & 14.03 & 12.98 & 12.56 & 68.2 \\
\hline 5.96 & +494814.4 & 18.79 & 17.77 & 17.33 & 15.89 & 14.86 & 14.47 & 20.0 \\
\hline .77 & +493 & 18.29 & 17.05 & 16.93 & 15.16 & 14.19 & 13.74 & 9.4 \\
\hline 204033.77 & +394838.8 & 18.85 & 17.26 & 17.70 & 01 & 14.07 & 13.85 & 17.5 \\
\hline 204053.55 & +395107.2 & 18.34 & 16.69 & 17.14 & 14 & 13.61 & 13.31 & 18.1 \\
\hline 204127.03 & +393730.0 & 17.02 & 15.85 & 15.61 & 13.90 & 13.00 & 12.70 & 14.1 \\
\hline 204308.84 & +424608.5 & 16.36 & 15.26 & 15.30 & 13.10 & 12.27 & 11.90 & 31.3 \\
\hline 204402.83 & +3931 16.3 & 17.77 & 16.36 & 16.45 & 14.34 & 13.55 & 13.25 & 29.7 \\
\hline 204433.07 & +431321.6 & 18.51 & 16.74 & 16.84 & 13.52 & 12.49 & 11.96 & 27.6 \\
\hline 204533.53 & +410727.8 & 18.01 & 16.13 & 16.64 & 13.63 & 12.68 & 12.26 & 19.0 \\
\hline 204546.40 & +455808.4 & 18.30 & 16.49 & 17.12 & 14.04 & 13.15 & 12.73 & 12.0 \\
\hline 204555.86 & +461201.9 & 19.28 & 18.29 & 18.32 & 16.43 & 15.39 & 15.03 & 10.7 \\
\hline 204648.46 & +441114.3 & 18.89 & 16.80 & 17.29 & 13.53 & 12.46 & 11.95 & 21.1 \\
\hline 204832.87 & +441159.7 & 17.89 & 16.39 & 16.16 & 14.16 & 13.23 & 12.75 & 21.9 \\
\hline 205021.10 & +465544.3 & 18.39 & 17.17 & 17.37 & 15.06 & 13.95 & 13.39 & 6.2 \\
\hline 205054.47 & +434623.8 & 16.51 & 15.61 & 15.27 & 14.00 & 13.21 & 12.97 & 28.8 \\
\hline 205112.11 & +472625.5 & 18.64 & 17.50 & 17.37 & 15.68 & 14.55 & 14.25 & 15.5 \\
\hline 205215.45 & +442810.8 & 16.82 & 15.48 & 15.47 & 13.20 & 12.21 & 11.73 & 9.6 \\
\hline 205225.79 & +461849.5 & 18.57 & 17.51 & 17.33 & 15.42 & 14.41 & 13.91 & 33.5 \\
\hline 205456.93 & +443924.5 & 17.85 & 16.21 & 16.59 & 14.11 & 13.21 & 12.81 & 8.7 \\
\hline
\end{tabular}


R. L. M. Corradi et al.: IPHAS and the symbiotic stars. I., Online Material p 5

Table 1. continued.

\begin{tabular}{|c|c|c|c|c|c|c|c|c|}
\hline \multicolumn{2}{|c|}{ RA $(J 2000.0)$ Dec } & $r$ & $i$ & $\mathrm{H} \alpha$ & $\bar{J}$ & $\bar{H}$ & $\overline{K_{\mathrm{s}}}$ & $d_{4}$ \\
\hline 205726.70 & +433529.6 & 17.22 & 15.78 & 15.93 & 13.41 & 12.40 & 12.01 & 16.1 \\
\hline 205755.03 & +525554.9 & 16.89 & 15.57 & 15.64 & 13.62 & 12.60 & 12.09 & 33.7 \\
\hline 0005.43 & +521705.1 & 15.54 & 14.46 & 14.34 & 13.06 & 12.06 & 1.63 & 8.7 \\
\hline 210024.07 & +521450.8 & 17.97 & 16.46 & 16.27 & 13.99 & 13.04 & 12.62 & 8.4 \\
\hline 210058.39 & +522856.3 & 17.41 & 15.87 & 16.27 & 12.33 & 11.08 & 10.48 & 6.5 \\
\hline 210135.60 & +495254.0 & 17.62 & 16.08 & 16.55 & 13.49 & 12.19 & 11.71 & 28.4 \\
\hline 210351.54 & +480335.1 & 18.95 & 17.05 & 17.72 & 15.22 & 14.53 & 14.43 & 19.7 \\
\hline 211551.96 & +513620.8 & 19.37 & 17.77 & 18.27 & 15.67 & 14.76 & 14.31 & 16.8 \\
\hline 211615.72 & +433658.4 & 19.44 & 17.52 & 18.10 & 15.52 & 14.81 & 14.51 & 34.3 \\
\hline 211922.95 & +464736.0 & 18.29 & 16.34 & 16.75 & 13.72 & 12.71 & 12.32 & 2.8 \\
\hline 211939.41 & +514412.6 & 18.56 & 17.01 & 17.22 & 14.78 & 13.90 & 13.51 & 5.7 \\
\hline 212054.77 & +465236.9 & 16.05 & 14.66 & 14.74 & 12.38 & 11.50 & 11.09 & 10.0 \\
\hline 212227.33 & +5235 55.7 & 19.21 & 17.67 & 17.86 & 15.19 & 13.91 & 13.25 & 23.7 \\
\hline 212240.17 & +465355.6 & 17.20 & 15.70 & 16.01 & 13.77 & 12.86 & 12.42 & 6.8 \\
\hline 212651.93 & +473413.9 & 16.85 & 15.43 & 15.54 & 13.41 & 12.51 & 12.15 & 13.8 \\
\hline 212820.10 & +552847.9 & 18.16 & 17.41 & 17.01 & 16.30 & 15.35 & 15.03 & 20.1 \\
\hline 212856.65 & +474742.0 & 15.92 & 14.98 & 14.50 & 13.29 & 12.56 & 12.39 & 27.9 \\
\hline 213516.28 & +572822.2 & 17.27 & 15.87 & 16.02 & 14.01 & 13.07 & 12.67 & 8.6 \\
\hline 213811.37 & +585317.2 & 18.54 & 17.02 & 17.49 & 14.70 & 13.63 & 13.24 & 35.6 \\
\hline 213935.62 & +57 1821.9 & 17.69 & 16.38 & 16.33 & 14.50 & 13.58 & 13.22 & 6.4 \\
\hline 69 & +57163 & 18.06 & 16.65 & 16. & & & .73 & 6.9 \\
\hline 213956.13 & +572707.9 & 19.42 & 17.74 & 17.48 & 15.51 & 14.64 & 14.22 & 1.6 \\
\hline 214020.05 & +575044.3 & 19.46 & 17.12 & 18.13 & 14.49 & 13.58 & 13.27 & 10.2 \\
\hline 214025.37 & +573416.2 & 18.51 & 16.96 & 16.79 & 14.90 & 14.03 & 13.61 & 5.0 \\
\hline 4027.32 & +581421.3 & 18.22 & 16.78 & 16.65 & 14.30 & 13.30 & 12.88 & 3.3 \\
\hline 21403 & +575833.2 & 18.80 & 17.15 & 17.56 & .90 & .99 & 13.62 & 11.7 \\
\hline 214114.98 & +573814.8 & 17.24 & 15.64 & 16.11 & 13.14 & 12.32 & 11.92 & 7.2 \\
\hline 214216.79 & +573622.0 & 17.13 & 15.46 & 15.99 & 13.08 & 12.28 & 11.95 & 11.8 \\
\hline 215408.13 & +594535.3 & 18.90 & 17.22 & 17.50 & 15.13 & 14.31 & 14.04 & 14.0 \\
\hline 22061 & +590207.1 & 16.12 & 14.59 & 14.63 & .00 & .10 & .74 & 13.0 \\
\hline 220754.48 & +590657.1 & 15.46 & 13.88 & 14.29 & 12.30 & 11.50 & 11.24 & 8.4 \\
\hline 221214.28 & +611246.4 & 18.92 & 17.23 & 17.58 & 15.10 & 14.28 & 13.89 & 18.6 \\
\hline 221430.15 & +613213.2 & 18.45 & 16.94 & 17.33 & 14.52 & 13.46 & 12.93 & 5.8 \\
\hline 22143 & +612758.7 & 17.73 & 16.04 & 16.58 & 13.70 & 12.60 & 12.15 & 2.5 \\
\hline 22145 & +614 & 16.37 & 15.24 & 15.38 & 13.39 & 12.48 & 12.15 & 11.0 \\
\hline 221530.21 & +604609.7 & 18.75 & 17.20 & 17.00 & 69 & 13.73 & 13.33 & 7.8 \\
\hline 221554.21 & +521727.5 & 18.12 & 17.32 & 17.07 & 15.86 & 14.99 & 14.82 & 8.3 \\
\hline 221600.81 & +591951.0 & 16.76 & 15.20 & 15.47 & 13.21 & 12.41 & 12.13 & 21.6 \\
\hline 221601.12 & +561450.5 & 18.35 & 16.80 & 17.10 & 14.51 & 3.61 & 13.25 & 11.2 \\
\hline 221606.52 & +522722.5 & 18.86 & 17.92 & 17.66 & 16.33 & 15.06 & .42 & 5.9 \\
\hline 221624.48 & +615919.0 & 18.60 & 17.09 & 17.51 & 14.61 & 13.53 & 13.12 & 18.1 \\
\hline 222535.13 & +625816.8 & 17.45 & 16.23 & 16.31 & 14.16 & 13.28 & 12.97 & 25.5 \\
\hline 222628.63 & +612049.0 & 18.68 & 18.13 & 16.55 & 14.62 & 13.75 & 13.42 & 24.2 \\
\hline 222843.00 & +622133.7 & 17.51 & 16.23 & 16.44 & 14.21 & 13.31 & 12.90 & 20.4 \\
\hline 224547.21 & +575053.9 & 15.87 & 14.88 & 14.90 & 09 & 12.13 & 11.68 & 8.0 \\
\hline 224604.51 & +605346.0 & 15.75 & 14.60 & 14.77 & 12.82 & 11.88 & 11.42 & 13.9 \\
\hline 224727.94 & +605853.5 & 18.50 & 16.87 & 17.24 & 14.39 & 13.42 & 12.97 & 13.1 \\
\hline 224734.41 & +580717.7 & 18.18 & 17.12 & 17.12 & 15.52 & 14.61 & 14.44 & 10.5 \\
\hline 224748.64 & +632155.1 & 17.34 & 15.91 & 16.28 & 13.62 & 12.58 & 12.10 & 29.7 \\
\hline 224759.08 & +615126.4 & 19.10 & 17.22 & 17.33 & 14.51 & 13.51 & 13.10 & 15.7 \\
\hline 225022.14 & +615328.9 & 17.16 & 16.27 & 16.05 & 14.99 & 14.04 & 13.72 & 12.0 \\
\hline 225143.33 & +625803.0 & 18.59 & 16.85 & 17.36 & 14.70 & 13.87 & 13.52 & 13.8 \\
\hline 225201.54 & +570322.4 & 19.08 & 17.81 & 17.92 & 15.80 & 14.79 & 14.28 & 21.4 \\
\hline 225318.88 & +62 2927.0 & 17.26 & 15.85 & 15.82 & 13.31 & 12.09 & 11.47 & 5.8 \\
\hline 225319.03 & +622631.7 & 18.29 & 16.79 & 17.13 & 14.13 & 13.08 & 12.62 & 7.6 \\
\hline 225345.86 & +595216.4 & 17.98 & 16.24 & 16.81 & 13.12 & 12.01 & 11.47 & 20.7 \\
\hline 225359.68 & +623749.7 & 16.31 & 15.11 & 15.27 & 12.99 & 11.92 & 11.38 & 1.3 \\
\hline 225407.90 & +623558.6 & 17.69 & 16.22 & 16.64 & 14.07 & 13.08 & 12.76 & 2.4 \\
\hline 225458.16 & +624132.7 & 18.07 & 16.45 & 16.84 & 14.12 & 13.05 & 12.55 & 3.6 \\
\hline 225528.19 & +570818.2 & 18.39 & 17.33 & 17.22 & 15.40 & 14.38 & 13.88 & 16.9 \\
\hline 225538.34 & +630231.6 & 17.47 & 16.19 & 16.48 & 14.16 & 13.17 & 12.79 & 6.6 \\
\hline
\end{tabular}


R. L. M. Corradi et al.: IPHAS and the symbiotic stars. I., Online Material p 6

Table 1. continued.

\begin{tabular}{|c|c|c|c|c|c|c|c|c|}
\hline \multicolumn{2}{|c|}{ RA (J2000.0) Dec } & $r$ & $i$ & $\mathrm{H} \alpha$ & $J$ & $\bar{H}$ & $\overline{K_{\mathrm{s}}}$ & $d_{4}$ \\
\hline 225556.25 & +624540.5 & 17.25 & 15.91 & 15.86 & 13.86 & 12.80 & 12.34 & 2.3 \\
\hline 225559.62 & +624744.8 & 16.09 & 14.75 & 15.03 & 12.65 & 11.60 & 11.11 & 3.4 \\
\hline 225614.14 & +624343.5 & 18.60 & 16.94 & 16.98 & 14.46 & 13.41 & 12.97 & 2.1 \\
\hline 225615.81 & +623932.8 & 19.17 & 17.02 & 17.34 & 14.44 & 13.53 & 13.07 & 2.4 \\
\hline 225630.95 & +624138.6 & 18.78 & 17.08 & 17.40 & 14.36 & 13.20 & 12.60 & 2.1 \\
\hline 225702.73 & +622901.9 & 19.29 & 17.45 & 17.31 & 14.89 & 13.84 & 13.36 & 10.6 \\
\hline 225834.40 & +613838.5 & 18.74 & 16.75 & 17.57 & 14.06 & 12.88 & 12.31 & 20.5 \\
\hline 225915.83 & +635224.2 & 19.01 & 17.28 & 17.82 & 14.68 & 13.53 & 12.99 & 27.3 \\
\hline 230023.89 & +624851.6 & 17.70 & 16.24 & 16.43 & 14.04 & 13.04 & 12.70 & 5.4 \\
\hline 230026.92 & +624830.9 & 17.66 & 16.32 & 16.61 & 14.09 & 13.21 & 12.80 & 5.3 \\
\hline 230029.03 & +624414.0 & 18.09 & 16.77 & 16.96 & 14.53 & 13.46 & 13.05 & 7.1 \\
\hline 230035.60 & +61 1730.6 & 18.54 & 17.31 & 17.37 & 15.06 & 13.99 & 13.50 & 9.1 \\
\hline 230111.35 & +625325.3 & 18.90 & 17.10 & 17.65 & 14.51 & 13.58 & 13.19 & 6.9 \\
\hline 230144.02 & +630616.5 & 18.77 & 17.14 & 17.64 & 14.59 & 13.61 & 13.11 & 7.8 \\
\hline 230202.60 & +640322.7 & 18.59 & 17.05 & 17.34 & 14.88 & 13.92 & 13.50 & 20.1 \\
\hline 230216.34 & +565755.6 & 18.95 & 17.89 & 17.74 & 16.13 & 14.71 & 14.06 & 19.7 \\
\hline 230218.37 & 619.5 & 17.27 & 15.54 & 16.15 & 13.21 & 12.15 & 11.70 & 10.1 \\
\hline 230247.08 & +64 4805.7 & 18.26 & 16.91 & 16.65 & 14.65 & 13.58 & 13.03 & 31.4 \\
\hline 230341.77 & +614736.1 & 19.11 & 17.20 & 17.71 & 14.31 & 12.85 & 12.07 & 4.8 \\
\hline 230501.79 & +600454.9 & 18.75 & 17.49 & 17.78 & 15.56 & 14.63 & 14.31 & 10.4 \\
\hline .93 & 20.1 & 18.62 & 16.93 & 17.39 & 51 & 48 & 3.05 & 19.8 \\
\hline 230531.15 & +60 1223.9 & 19.10 & 17.58 & 17.91 & 15.18 & 14.08 & 13.64 & 6.8 \\
\hline 230546.69 & +635017.1 & 18.64 & 17.15 & 17.46 & 14.80 & 13.62 & 13.00 & 26.5 \\
\hline 231244.96 & +614605.4 & 14.64 & 13.94 & 13.66 & 12.77 & 12.04 & 11.72 & 10.1 \\
\hline 49.78 & +592054.5 & 19.01 & 17.57 & 17.78 & 15.51 & 14.70 & 14.43 & 21.4 \\
\hline .92 & 44.1 & 17.12 & 15.91 & 15.87 & .13 & .21 & 2.90 & 12.8 \\
\hline 231411.67 & +62 1800.0 & 18.28 & 16.65 & 17.08 & 14.66 & 13.82 & 13.51 & 8.8 \\
\hline 231627.57 & +630338.1 & 19.02 & 17.70 & 17.18 & 15.15 & 14.03 & 13.63 & 22.2 \\
\hline 232450.86 & +633815 & 18.73 & 17.09 & 17.38 & 14.79 & 13.94 & 13.81 & 24.1 \\
\hline 232744.89 & +61 & 18.31 & 17.21 & 17.26 & 15.56 & .47 & 1.18 & 18.6 \\
\hline 233630.38 & +634816.4 & 18.56 & 17.09 & 17.08 & 14.76 & 13.88 & 13.44 & 30.5 \\
\hline 234628.69 & +632802.9 & 17.18 & 15.67 & 15.96 & 13.59 & 12.64 & 12.21 & 6.4 \\
\hline 234641.72 & +665316.6 & 17.29 & 16.01 & 15.56 & 13.88 & 12.91 & 12.51 & 56.0 \\
\hline 234740.63 & 217.5 & 17.16 & 15.53 & 15.91 & 13.61 & 12.70 & 12.32 & 7.2 \\
\hline 235530.14 & +655657.0 & 17.05 & 15.07 & 15.78 & 12.65 & 11.85 & 11.52 & 24.4 \\
\hline \multicolumn{9}{|c|}{ Part B: D-type candidates } \\
\hline 000213.37 & +645424.6 & 16.97 & 16.11 & 15.62 & 10.66 & 9.67 & 9.05 & 20.2 \\
\hline .16 & +672 & 16.48 & 14.92 & 15.42 & 12.04 & 10.86 & 10.02 & 47.3 \\
\hline 2.31 & +58 & 17.36 & 16.02 & 15.41 & 13.63 & 12.86 & 12.35 & 48.2 \\
\hline 000718.66 & +653642.1 & 16.59 & 15.17 & 15.30 & 12.82 & 11.76 & 11.11 & 6.0 \\
\hline 000936.91 & +664545.4 & 19.43 & 18.02 & 17.90 & 15.71 & 14.53 & 13.70 & 23.5 \\
\hline 1020.53 & +583708.6 & 19.36 & 18.20 & 17.02 & 15.74 & 14.28 & 13.22 & 7.3 \\
\hline .20 & +663 & 17.08 & 15.71 & 15.64 & 13.34 & 12.46 & 11.91 & 27.2 \\
\hline .30 & +584 & 17.80 & 15.98 & 16.28 & 13.47 & 12.42 & 11.71 & 3.9 \\
\hline 001102.24 & +584232.2 & 18.46 & 16.48 & 17.05 & 86 & 13.11 & 12.61 & 3.3 \\
\hline 001125.46 & +584209.6 & 18.19 & 16.15 & 16.83 & 14.02 & 13.19 & 12.76 & 5.1 \\
\hline 01259.13 & +654700.7 & 18.08 & 16.82 & 16.72 & 15.54 & 13.74 & 12.42 & 11.0 \\
\hline 001414.65 & +610009.6 & 18.39 & 17.34 & 17.43 & 15.35 & 14.53 & 13.87 & 21.2 \\
\hline 001636.19 & +633348.6 & 19.32 & 18.12 & 18.33 & 16.06 & 14.87 & 14.08 & 17.0 \\
\hline 001643.60 & +643138.7 & 17.98 & 16.85 & 16.77 & 14.91 & 13.98 & 13.48 & 17.7 \\
\hline & +654846.5 & 18.62 & 17.26 & 17.40 & 14.88 & 13.68 & 12.99 & 15.6 \\
\hline 001853.83 & +62 2013.3 & 18.59 & 17.41 & 17.42 & 15.27 & 14.41 & 13.91 & 15.2 \\
\hline 001903.83 & +624225.9 & 19.46 & 18.37 & 18.36 & 16.35 & 15.53 & 14.79 & 12.2 \\
\hline 002143.02 & +614752.4 & 18.37 & 17.24 & 17.39 & 15.13 & 14.18 & 13.61 & 13.5 \\
\hline 002208.28 & +612545.3 & 19.27 & 18.14 & 18.19 & 16.44 & 15.56 & 15.02 & 13.8 \\
\hline 002457.77 & +655134.1 & 19.28 & 17.70 & 18.15 & 14.49 & 13.33 & 12.57 & 19.8 \\
\hline 002630.19 & +655145.8 & 18.77 & 17.30 & 17.22 & 14.95 & 13.80 & 12.96 & 19.2 \\
\hline 002820.89 & +650926.3 & 19.30 & 17.21 & 17.77 & 14.93 & 14.14 & 13.72 & 16.3 \\
\hline 003013.46 & +652812.5 & 18.84 & 17.42 & 17.53 & 14.62 & 12.88 & 11.69 & 4.5 \\
\hline & +585919.4 & 15.82 & 14.92 & 14.51 & 13.73 & 12.61 & 11.73 & 72.2 \\
\hline 003617.42 & +661513.3 & 18.16 & 16.71 & 16.60 & 14.35 & 13.50 & 12.80 & 29.6 \\
\hline
\end{tabular}


Table 1. continued.

\begin{tabular}{|c|c|c|c|c|c|c|c|c|}
\hline \multicolumn{2}{|c|}{ RA (J2000.0) Dec } & $r$ & $i$ & $\mathrm{H} \alpha$ & $\bar{J}$ & $\bar{H}$ & $\overline{K_{\mathrm{s}}}$ & $d_{4}$ \\
\hline 003756.79 & +630459.1 & 17.57 & 16.63 & 15.48 & 14.56 & 13.59 & 12.97 & 20.2 \\
\hline 003814.97 & +630559.7 & 15.84 & 14.85 & 14.59 & 12.50 & 11.48 & 10.64 & 20.3 \\
\hline 4224.91 & +66 5931.9 & 19.47 & 18.13 & 18.31 & 16.29 & 15.12 & 14.24 & 37.6 \\
\hline 004232.04 & +614757.5 & 19.36 & 17.29 & 17.92 & 14.69 & 13.56 & 12.86 & 9.0 \\
\hline 004234.61 & +61 1716.1 & 16.76 & 15.60 & 15.78 & 13.69 & 12.74 & 12.23 & 4.4 \\
\hline 004254.48 & +61 1931.2 & 16.89 & 15.46 & 15.33 & 12.53 & 11.15 & 9.98 & 4.3 \\
\hline 004255.80 & +612347.0 & 18.39 & 16.95 & 16.91 & 14.32 & 13.24 & 12.54 & 5.3 \\
\hline 004302.63 & +610923.0 & 18.55 & 16.89 & 17.10 & 14.32 & 13.31 & 12.73 & 7.2 \\
\hline 004315.16 & +612501.4 & 17.72 & 16.27 & 16.61 & 14.29 & 13.27 & 12.70 & 6.8 \\
\hline 004417.91 & +620118.0 & 18.60 & 16.63 & 16.93 & 13.83 & 13.08 & 12.61 & 5.4 \\
\hline 004420.30 & +615859.2 & 17.10 & 15.56 & 15.98 & 13.58 & 12.69 & 12.18 & 5.6 \\
\hline 4420.78 & +615834.1 & 17.96 & 16.27 & 16.66 & 13.77 & 12.84 & 12.27 & 5.9 \\
\hline 004446.83 & +66 5706.3 & 18.44 & 17.26 & 17.21 & 15.35 & 14.48 & 13.67 & 40.9 \\
\hline 005443.63 & +610904.0 & 19.38 & 17.65 & 18.15 & 14.30 & 13.15 & 12.47 & 33.5 \\
\hline 5449.44 & +633542.6 & 19.26 & 17.98 & 17.90 & 15.34 & 14.13 & 13.03 & 31.7 \\
\hline 930.32 & 54.8 & 15.87 & 14.71 & 14.90 & 12.82 & 11.93 & 11.33 & 41.4 \\
\hline 010316.60 & 701.1 & 15.30 & 14.33 & 14.22 & 12.18 & 11.26 & 10.70 & 43.9 \\
\hline 010722.27 & +5943 35.2 & 19.38 & 18.16 & 18.29 & 16.48 & 15.68 & 14.92 & 45.8 \\
\hline 011220.50 & +643917.2 & 17.07 & 15.63 & 15.91 & 13.30 & 12.17 & 11.48 & 16.5 \\
\hline 011935.82 & +625525.8 & 17.18 & 15.91 & 16.01 & 13.64 & 12.46 & 11.55 & 36.0 \\
\hline 2107.17 & +612919.5 & 18.13 & 16.78 & 16. & 4.02 & .03 & 12.47 & 8.6 \\
\hline 012109.27 & +612652.7 & 17.12 & 16.18 & 16.14 & 14.26 & 13.15 & 12.51 & 8.8 \\
\hline 012158.27 & +613018.6 & 18.22 & 16.77 & 16.84 & 14.50 & 13.51 & 12.85 & 8.0 \\
\hline 012444.28 & +615329.9 & 18.80 & 17.35 & 17.31 & 14.60 & 13.45 & 12.74 & 17.7 \\
\hline 2544.66 & +613611.7 & 18.87 & 18.20 & 16.86 & 15.48 & 13.22 & 11.49 & 21.5 \\
\hline 3720.01 & 57.7 & 17.47 & 16.01 & 15.51 & 3.62 & .69 & 12.08 & 28.1 \\
\hline 013913.79 & +622145.3 & 17.84 & 16.48 & 16.54 & 13.79 & 12.48 & 11.51 & 30.5 \\
\hline 014203.40 & +612052.9 & 18.68 & 17.54 & 17.53 & 15.29 & 14.14 & 13.41 & 30.0 \\
\hline 4349.54 & +622952.6 & 19.33 & 17.97 & 18.02 & 15.44 & 14.14 & 13.26 & 17.7 \\
\hline .50 & +64 1216.5 & 19.36 & 18.28 & 17.99 & 15.91 & .94 & 14.30 & 13.6 \\
\hline 014550.34 & +573403.1 & 16.23 & 15.59 & 15.29 & 12.49 & 10.92 & 9.72 & 39.8 \\
\hline 014551.20 & +641605.5 & 19.03 & 17.86 & 17.34 & 15.65 & 14.50 & 13.28 & 17.5 \\
\hline 20039.48 & +603259.1 & 14.73 & 14.12 & 12.79 & 12.45 & 11.26 & 9.88 & 33.6 \\
\hline 8.00 & +593732.6 & 17.95 & 16.77 & 16.93 & 14.57 & 13.35 & 12.53 & 27.4 \\
\hline 021623.72 & +601657.6 & 19.28 & 17.98 & 18.05 & 15.56 & 1.47 & 13.65 & 30.6 \\
\hline 021745.54 & +660022.3 & 17.73 & 16.54 & 16.44 & 14.37 & 13.17 & 12.31 & 33.3 \\
\hline 022103.83 & +620958.4 & 18.74 & 17.45 & 17.65 & 15.36 & 14.32 & 13.66 & 26.5 \\
\hline 2106.39 & +590113.6 & 17.03 & 16.02 & 16.08 & 13.77 & 12.46 & 11.32 & 41.0 \\
\hline 02222 & +612 & 18.48 & 16.95 & 16.98 & 14.17 & 12.85 & 11.99 & 31.8 \\
\hline 022525.64 & +62 1157.7 & 18.69 & 17.33 & 17.63 & 15.17 & 14.01 & 13.25 & 17.1 \\
\hline 022622.40 & +613308.7 & 18.35 & 17.05 & 17.18 & 14.64 & 13.49 & 12.73 & 17.2 \\
\hline 022639.54 & +620240.0 & 18.21 & 17.00 & 17.07 & 14.90 & 13.85 & 13.20 & 11.8 \\
\hline 022653.83 & +600543.3 & 18.94 & 17.17 & 17.69 & 14.78 & 13.91 & 13.41 & 10.4 \\
\hline 022743.09 & +581 & 17.87 & 16.93 & 16.95 & 15.17 & 14.30 & 13.74 & 36.3 \\
\hline 022902.78 & +611527.7 & 17.76 & 16.46 & 16.69 & .24 & .18 & 12.57 & 10.3 \\
\hline 022914.18 & +613325.5 & 17.81 & 16.52 & 16.83 & 14.37 & 13.28 & 12.59 & 9.1 \\
\hline 022924.29 & +611354.9 & 18.29 & 16.83 & 17.13 & 14.41 & 13.21 & 12.48 & 8.7 \\
\hline 022935.91 & +611556.8 & 13.83 & 12.98 & 12.76 & 11.14 & 9.91 & 8.94 & 9.2 \\
\hline 023024.71 & +563657.6 & 17.08 & 16.25 & 16.18 & 14.68 & 13.90 & 13.42 & 63.4 \\
\hline 023102.00 & +610757.2 & 17.84 & 16.45 & 16.76 & 13.88 & 12.67 & 11.81 & 10.2 \\
\hline 023109.66 & +60 5438.9 & 18.71 & 17.30 & 17.62 & 14.14 & 13.03 & 12.33 & 9.5 \\
\hline 023410.28 & +612440.4 & 13.64 & 12.97 & 12.46 & 11.44 & 10.41 & 9.51 & 9.3 \\
\hline 023425.20 & +622108.9 & 18.27 & 17.06 & 17.23 & 14.88 & 13.70 & 12.83 & 19.0 \\
\hline 023450.40 & +622518.5 & 18.96 & 17.68 & 17.64 & 15.48 & 14.60 & 14.00 & 20.1 \\
\hline 023503.66 & +615452.4 & 16.78 & 15.83 & 15.89 & 14.06 & 13.17 & 12.57 & 12.2 \\
\hline 023515.33 & +614803.3 & 16.75 & 15.67 & 15.63 & 13.54 & 12.51 & 11.77 & 9.1 \\
\hline 023823.48 & +615105.2 & 18.09 & 17.06 & 17.17 & 15.26 & 14.33 & 13.86 & 12.0 \\
\hline 023828.34 & +594001.3 & 18.85 & 17.71 & 17.76 & 15.89 & 14.96 & 14.23 & 18.2 \\
\hline 023940.51 & +590401.6 & 17.54 & 16.23 & 16.38 & 13.86 & 12.69 & 11.91 & 22.1 \\
\hline 024135.93 & +573738.0 & 18.82 & 17.88 & 17.68 & 16.42 & 15.14 & 13.30 & 7.0 \\
\hline 024147.73 & +601109.1 & 16.13 & 14.79 & 14.99 & 12.53 & 11.83 & 11.26 & 19.3 \\
\hline
\end{tabular}


R. L. M. Corradi et al.: IPHAS and the symbiotic stars. I., Online Material p 8

Table 1. continued.

\begin{tabular}{|c|c|c|c|c|c|c|c|c|}
\hline \multicolumn{2}{|c|}{ RA (J2000.0) Dec } & $r$ & $i$ & $\mathrm{H} \alpha$ & $J$ & $\bar{H}$ & $\overline{K_{\mathrm{s}}}$ & $d_{4}$ \\
\hline 024326.34 & +590631.1 & 16.64 & 15.57 & 15.39 & 13.68 & 12.73 & 12.20 & 23.7 \\
\hline 024439.54 & +605954.8 & 16.24 & 15.22 & 15.11 & 13.17 & 12.10 & 11.25 & 9.1 \\
\hline 024442.09 & +603427.5 & 19.05 & 17.71 & 17.95 & 15.67 & 14.44 & 13.78 & 9.0 \\
\hline 024507.00 & +610744.0 & 19.01 & 17.60 & 17.69 & 15.52 & 14.35 & 13.63 & 10.5 \\
\hline 024748.61 & +605750.4 & 14.02 & 13.49 & 13.14 & 12.04 & 10.94 & 9.96 & 23.8 \\
\hline 024834.20 & +60 2755.5 & 18.64 & 17.41 & 17.57 & 15.24 & 14.16 & 13.30 & 17.6 \\
\hline 024949.77 & +601715.2 & 18.39 & 17.13 & 16.96 & 15.03 & 13.81 & 13.04 & 10.7 \\
\hline 024953.35 & +601328.3 & 16.78 & 15.77 & 15.51 & 13.72 & 12.73 & 11.95 & 11.2 \\
\hline 025151.96 & +601309.3 & 18.73 & 17.53 & 17.44 & 15.33 & 14.31 & 13.71 & 4.8 \\
\hline 025158.30 & +60 2359.2 & 18.49 & 17.58 & 17.29 & 15.39 & 14.36 & 13.57 & 4.8 \\
\hline 025207.87 & +601455.6 & 17.41 & 16.35 & 16.48 & 14.37 & 13.35 & 12.57 & 4.2 \\
\hline 25338.45 & +604116.0 & 18.68 & 17.64 & 17.40 & 15.36 & 14.45 & 13.87 & 3.9 \\
\hline 025356.29 & +603920.7 & 18.16 & 17.11 & 16.79 & 15.04 & 14.09 & 13.39 & 2.1 \\
\hline 025400.78 & +603639.5 & 19.43 & 18.14 & 18.41 & 16.05 & 15.24 & 14.82 & 2.0 \\
\hline 025406.19 & +603833.5 & 17.79 & 16.71 & 16.85 & 14.89 & 13.73 & 12.85 & 1.6 \\
\hline 025417.67 & 314.7 & 18.52 & 17.39 & 17.27 & 15.61 & 14.61 & 13.88 & 1.8 \\
\hline 025456.86 & +603813.7 & 19.45 & 18.23 & 18.37 & 15.86 & 15.06 & 14.54 & 2.2 \\
\hline 025545.29 & +590438.2 & 17.25 & 16.50 & 16.28 & 14.43 & 12.91 & 11.65 & 30.8 \\
\hline 025602.38 & +611159.9 & 17.91 & 16.58 & 16.85 & 13.90 & 12.62 & 11.62 & 12.7 \\
\hline 025720.41 & +604446.6 & 19.32 & 17.91 & 17.95 & 15.58 & 14.70 & 14.19 & 6.7 \\
\hline 5721.57 & +60 & 19.02 & 17.62 & 17.66 & 07 & 12.81 & .79 & 8.1 \\
\hline 025757.37 & +602040.7 & 17.55 & 16.50 & 16.39 & 14.42 & 13.57 & 13.02 & 8.4 \\
\hline 025824.52 & +603416.4 & 18.51 & 17.36 & 17.25 & 15.41 & 14.62 & 14.13 & 4.3 \\
\hline 025851.22 & +603954.5 & 18.68 & 17.40 & 17.49 & 15.22 & 14.18 & 13.49 & 5.1 \\
\hline 025902.08 & +603624.4 & 17.60 & 16.60 & 16.23 & 14.70 & 13.73 & 13.07 & 3.9 \\
\hline 5925.87 & +60 & 19.19 & 17.96 & 18.20 & .58 & .45 & 3.83 & 1.8 \\
\hline 025940.84 & +603453.6 & 18.37 & 17.23 & 16.99 & 15.07 & 13.99 & 13.29 & 1.7 \\
\hline 025942.81 & +602821.2 & 18.27 & 17.27 & 17.07 & 15.59 & 14.65 & 13.95 & 2.0 \\
\hline 0105.20 & +603155.4 & 15.85 & 14.77 & 14.91 & 12.85 & 11.90 & 11.29 & 3.0 \\
\hline 34.36 & +60 & 19.30 & 17.60 & 17.99 & 4.78 & .43 & 2.56 & 3.1 \\
\hline 030411.04 & +605504.1 & 18.89 & 17.61 & 17.50 & 15.16 & 90 & 13.06 & 21.4 \\
\hline 030555.54 & +603709.4 & 18.79 & 17.41 & 17.40 & 15.00 & 13.81 & 13.02 & 22.9 \\
\hline 030614.52 & +583935.0 & 19.26 & 17.62 & 17.67 & 15.19 & 14.20 & 13.61 & 21.5 \\
\hline 030902.19 & +582 & 18.42 & 17.30 & 17.18 & 14.62 & 13.51 & 12.73 & 26.5 \\
\hline 031046.27 & +593 & 15.08 & 14.51 & 14.29 & 12.82 & 11.75 & 10.65 & 29.5 \\
\hline 031405.39 & +584453.4 & 19.00 & 17.66 & 17.61 & 15.70 & 14.65 & 14.02 & 26.5 \\
\hline 031653.16 & +600305.5 & 19.43 & 17.97 & 18.06 & 15.55 & 14.34 & 13.66 & 10.9 \\
\hline 53.27 & +601 & 18.05 & 16.61 & 16.77 & 13.34 & 12.12 & 11.25 & 12.8 \\
\hline 56.98 & +595 & 18.97 & 17.51 & 17.12 & 15.14 & 13.80 & 12.88 & 13.3 \\
\hline 031741.86 & +530850.2 & 18.15 & 17.04 & 17.11 & 15.04 & 14.04 & 13.40 & 42.0 \\
\hline 031807.44 & +590339.7 & 17.75 & 16.34 & 16.52 & 14.38 & 13.43 & 12.62 & 22.0 \\
\hline 031912.06 & +594009.5 & 18.78 & 17.14 & 17.59 & 15.41 & 14.29 & 13.53 & 18.4 \\
\hline 03203 & +601818.3 & 15.85 & 14.52 & 14.85 & 12.10 & 10.81 & 9.86 & 3.5 \\
\hline 032039.49 & +562 & 14.68 & 13.40 & 13.07 & 10.95 & 9.57 & 8.22 & 35.3 \\
\hline 032508.89 & +605654.6 & 19.28 & 18.76 & 18.26 & 16.87 & 15.50 & 14.44 & 26.5 \\
\hline 032536.55 & +560533.2 & 16. & 15. & 15.64 & 13.21 & 12.46 & 11.88 & 35.6 \\
\hline 032638.60 & +585125.1 & 18. & 17.04 & 17.20 & 14.22 & 13.00 & 12.31 & 10.6 \\
\hline 032755.71 & +581806.0 & 18.01 & 16.81 & 16.96 & 14.38 & 13.41 & 12.90 & 22.7 \\
\hline 032835.36 & +585656.0 & 18.48 & 17.23 & 16.84 & 14.59 & 13.57 & 13.02 & 12.9 \\
\hline 032857.92 & +584050.3 & 19.35 & 17.64 & 17.99 & 14.99 & 14.01 & 13.36 & 19.8 \\
\hline 033013.60 & +580423.6 & 18.60 & 16.98 & 17.35 & 14.17 & 12.92 & 12.12 & 23.1 \\
\hline 033132.99 & +603555.9 & 16.71 & 15.94 & 15.54 & 13.98 & 13.20 & 12.76 & 23.0 \\
\hline 033155.48 & +582232.7 & 18.24 & 16.64 & 16.96 & 13.95 & 12.81 & 12.21 & 19.6 \\
\hline 033202.36 & +593200.7 & 17.75 & 16.33 & 16.50 & 13.76 & 12.66 & 12.06 & 34.7 \\
\hline 033540.02 & +575835.7 & 18.37 & 16.76 & 17.22 & 14.35 & 12.94 & 11.93 & 25.4 \\
\hline 034513.72 & +543525.9 & 16.93 & 15.49 & 15.83 & 13.25 & 12.47 & 11.84 & 25.9 \\
\hline 034609.84 & +545550.8 & 17.59 & 16.18 & 16.50 & 14.10 & 13.33 & 12.76 & 25.9 \\
\hline 035001.78 & +530756.2 & 18.93 & 17.36 & 17.76 & 15.07 & 14.12 & 13.41 & 40.1 \\
\hline 035046.67 & +540109.3 & 16.87 & 15.34 & 15.67 & 13.14 & 11.90 & 11.09 & 21.3 \\
\hline & +534542.0 & 18.47 & 16.49 & 17.28 & 12.55 & 10.92 & 9.65 & 29.2 \\
\hline 035608.31 & +534526.0 & 15.78 & 14.18 & 14.73 & 11.62 & 10.73 & 10.17 & 28.9 \\
\hline
\end{tabular}


Table 1. continued.

\begin{tabular}{|c|c|c|c|c|c|c|c|c|}
\hline \multicolumn{2}{|c|}{ RA (J2000.0) Dec } & $r$ & $i$ & $\mathrm{H} \alpha$ & $J$ & $\bar{H}$ & $\overline{K_{\mathrm{s}}}$ & $d_{4}$ \\
\hline 035619.88 & +522944.0 & 19.41 & 18.00 & 18.19 & 15.43 & 14.61 & 14.09 & 14.9 \\
\hline 035634.20 & +514833.2 & 17.88 & 16.37 & 16.39 & 13.98 & 12.84 & 12.18 & 15.6 \\
\hline 5700.59 & +514734.8 & 16.33 & 15.40 & 14.79 & 13.29 & 12.41 & 11.91 & 13.6 \\
\hline 035707.52 & +574142.7 & 19.25 & 18.33 & 17.47 & 16.00 & 15.12 & 14.48 & 24.5 \\
\hline 035719.39 & +570743.1 & 18.91 & 17.82 & 17.92 & 15.72 & 14.82 & 14.15 & 5.1 \\
\hline 035823.95 & +522312.6 & 18.96 & 17.77 & 17.05 & 15.74 & 14.83 & 14.30 & 13.2 \\
\hline 035841.02 & +513723.9 & 19.30 & 18.00 & 18.01 & 15.85 & 14.71 & 13.80 & 9.6 \\
\hline 035923.18 & +514250.8 & 18.62 & 17.23 & 17.49 & 15.10 & 14.18 & 13.63 & 8.0 \\
\hline 040006.20 & +513902.3 & 17.48 & 16.47 & 16.29 & 14.16 & 13.23 & 12.63 & 9.8 \\
\hline 040056.06 & +584529.0 & 19.09 & 18.04 & 17.48 & 15.93 & 14.85 & 14.00 & 47.6 \\
\hline 040220.91 & +525101.9 & 18.83 & 17.84 & 17.48 & 15.81 & 14.80 & 14.21 & 23.3 \\
\hline 450.08 & +542744.8 & 18.43 & 17.23 & 17.30 & 14.95 & 13.82 & 12.79 & 27.6 \\
\hline 810.62 & +524258.6 & 18.07 & 17.01 & 16.67 & 14.95 & 13.86 & 13.18 & 16.6 \\
\hline 040930.70 & +524450.6 & 19.47 & 18.26 & 18.43 & 15.86 & 14.96 & 14.50 & 18.8 \\
\hline 040936.51 & +490306.8 & 19.05 & 17.97 & 18.07 & 15.74 & 14.65 & 13.96 & 68.2 \\
\hline+1011.85 & +505954.6 & 16.82 & 14.71 & 15.38 & 10.92 & 8.90 & 7.12 & 40.8 \\
\hline 7.61 & +524256.3 & 19.34 & 18.19 & 18.07 & 15.96 & 14.83 & 13.98 & 29.5 \\
\hline 042240.39 & +461746.3 & 18.66 & 17.51 & 17.33 & 15.24 & 14.15 & 13.42 & 47.5 \\
\hline 042256.11 & +534709.4 & 19.30 & 18.20 & 18.06 & 16.27 & 15.57 & 14.75 & 24.3 \\
\hline 042303.57 & +432651.0 & 18.37 & 17.68 & 17.25 & 16.34 & 15.62 & 15.06 & 53.9 \\
\hline .10 & +445346.7 & 19.43 & 18.16 & 18.04 & 16.22 & 15.16 & 14.44 & 37.6 \\
\hline 04250 & 57.7 & 18.10 & 16.61 & 16.85 & 82 & 2.99 & 12.49 & 43.3 \\
\hline 042754.38 & +481008.6 & 19.34 & 18.09 & 17.80 & 15.51 & 13.54 & 11.94 & 38.1 \\
\hline 042914.20 & +452434.8 & 17.03 & 16.05 & 15.82 & 14.47 & 13.46 & 12.68 & 8.7 \\
\hline 12926.85 & +451610.2 & 17.23 & 15.94 & 15.74 & 14.04 & 12.62 & 11.44 & 12.2 \\
\hline 2.43 & +534 & 18.53 & 17.22 & 16.70 & & .92 & 3.10 & 47.8 \\
\hline 043228.30 & +462017.1 & 19.33 & 17.79 & 17.68 & 15.48 & 14.63 & 14.19 & 44.6 \\
\hline 043236.68 & +424121.1 & 18.45 & 17.27 & 17.19 & 02 & 13.77 & 12.82 & 32.6 \\
\hline 043347.04 & +443132.7 & 18.45 & 17.32 & 17.32 & 15.38 & 14.30 & 13.53 & 26.0 \\
\hline 044112.44 & +471652.3 & 18.29 & 17.05 & 16.88 & 14.86 & 13.80 & 13.06 & 26.2 \\
\hline 044338.64 & +410941.5 & 15.02 & 14.02 & 13.83 & 12.25 & 11.19 & 10.17 & 42.7 \\
\hline 044539.97 & +420229.9 & 19.31 & 18.18 & 18.27 & 16.33 & 15.45 & 14.84 & 3.0 \\
\hline 044542.71 & +420213.8 & 18.67 & 17.20 & 17.26 & 14.68 & 13.46 & 12.53 & 3.2 \\
\hline 5432.84 & +432123.8 & 17.72 & 16.54 & 16.68 & 15.06 & 14.13 & 13.51 & 42.1 \\
\hline 625.15 & +434 & 18.13 & 17.13 & 16.29 & 15.25 & .08 & 13.04 & 36.8 \\
\hline 045717.54 & +402121.7 & 16.26 & 15.14 & 15.01 & 12.82 & 11.77 & 11.10 & 30.7 \\
\hline 050124.25 & +464847.7 & 15.07 & 13.80 & 14.06 & 12.30 & 11.22 & 9.99 & 36.6 \\
\hline 050219.46 & +480337.6 & 18.68 & 17.63 & 17.35 & 16.12 & 15.32 & 14.44 & 55.4 \\
\hline 0747.35 & +402355.3 & 18.57 & 17.69 & 17.12 & 15.43 & 14.45 & 13.62 & 29.0 \\
\hline 0.97 & +424212.5 & 17.57 & 16.58 & 16.25 & 14.88 & 13.90 & 13.24 & 25.6 \\
\hline 050805.09 & +402440.3 & 18.25 & 17.20 & 17.12 & 09 & 14.00 & .28 & 28.7 \\
\hline 050810.23 & +405351.8 & 19.17 & 18.30 & 17.17 & 16.46 & 15.60 & 15.07 & 36.9 \\
\hline 1348.76 & +324206.7 & 18.82 & 17.73 & 17.50 & 15.27 & 14.21 & 13.43 & 45.0 \\
\hline 052243.78 & +332525.8 & 16.51 & 15.09 & 15.24 & 12.38 & 10.77 & 9.42 & 4.2 \\
\hline 052254.80 & +332718.7 & 19.10 & 18.22 & 17.78 & 16.59 & 15.53 & 14.76 & 3.3 \\
\hline 052443.05 & +350123.3 & 18.42 & 17.28 & 17.13 & 15.11 & 14.10 & 13.50 & 17.2 \\
\hline 052514.61 & +431549.4 & 17.62 & 16.86 & 16.65 & 15.21 & 14.30 & 13.51 & 60.6 \\
\hline 052527.71 & +431407.1 & 18.14 & 17.28 & 16.94 & 15.17 & 14.20 & 13.43 & 59.1 \\
\hline 052610.73 & +333750.8 & 18.24 & 17.18 & 17.30 & 15.22 & 14.21 & 13.62 & 32.3 \\
\hline 052707.61 & +34 5724.8 & 18.66 & 17.66 & 17.24 & 15.33 & 14.11 & 13.13 & 7.2 \\
\hline 052707.83 & +345727.6 & 18.38 & 17.41 & 17.27 & 15.69 & 14.75 & 14.27 & 7.2 \\
\hline 052732.45 & +350240.7 & 17.22 & 16.36 & 16.30 & 14.56 & 13.54 & 12.90 & 9.4 \\
\hline 052742.63 & +344152.9 & 18.76 & 17.56 & 17.07 & 15.67 & 14.77 & 14.21 & 2.9 \\
\hline 052746.99 & +34 4401.1 & 18.77 & 17.83 & 17.64 & 15.95 & 15.24 & 14.85 & 2.7 \\
\hline 052808.59 & +342538.4 & 16.01 & 15.25 & 14.95 & 13.51 & 12.50 & 11.83 & 1.8 \\
\hline 052829.01 & +350138.8 & 18.92 & 17.82 & 17.36 & 15.66 & 14.85 & 14.31 & 6.5 \\
\hline 052829.06 & +310324.4 & 17.91 & 16.71 & 16.96 & 14.60 & 13.48 & 12.87 & 36.1 \\
\hline 052832.58 & +345922.6 & 18.98 & 17.90 & 17.96 & 16.15 & 15.12 & 14.50 & 7.4 \\
\hline 052857.93 & +350937.0 & 17.66 & 16.82 & 16.70 & 15.19 & 14.31 & 13.85 & 8.3 \\
\hline 053029.55 & +333220.8 & 18.48 & 17.12 & 17.39 & 15.44 & 14.35 & 13.74 & 9.6 \\
\hline 053113.16 & +382006.6 & 16.53 & 15.68 & 14.94 & 12.54 & 11.74 & 11.14 & 31.0 \\
\hline
\end{tabular}


Table 1. continued.

\begin{tabular}{|c|c|c|c|c|c|c|c|c|}
\hline \multicolumn{2}{|c|}{ RA (J2000.0) Dec } & $r$ & $i$ & $\mathrm{H} \alpha$ & $J$ & $\bar{H}$ & $\overline{K_{\mathrm{s}}}$ & $d_{4}$ \\
\hline 053204.48 & +301731.3 & 18.90 & 17.63 & 17.65 & 15.34 & 14.28 & 13.64 & 8.0 \\
\hline 053209.27 & +301853.0 & 18.68 & 17.37 & 17.40 & 15.20 & 14.25 & 13.37 & 8.3 \\
\hline 3309.32 & +325030.1 & 17.72 & 16.67 & 16.22 & 14.93 & 13.98 & 13.47 & 25.0 \\
\hline 053350.71 & +304254.0 & 17.62 & 16.32 & 16.50 & 14.10 & 12.88 & 12.16 & 32.4 \\
\hline 053423.83 & +253910.5 & 18.53 & 17.04 & 17.05 & 14.88 & 13.93 & 13.38 & 8.6 \\
\hline 053440.77 & +254238.2 & 17.48 & 16.28 & 15.63 & 14.09 & 12.12 & 10.58 & 11.3 \\
\hline 053517.98 & +274758.1 & 18.12 & 17.04 & 16.64 & 15.25 & 14.27 & 13.65 & 13.2 \\
\hline 053523.89 & +321721.7 & 17.93 & 16.77 & 16.97 & 15.01 & 14.09 & 13.60 & 11.2 \\
\hline 053538.79 & +322115.2 & 18.82 & 17.66 & 17.02 & 15.81 & 15.00 & 14.56 & 10.1 \\
\hline 053544.51 & +364940.1 & 19.08 & 17.78 & 18.00 & 15.64 & 14.74 & 14.12 & 30.6 \\
\hline 053611.80 & +273436.0 & 15.53 & 14.49 & 14.28 & 13.46 & 12.31 & 11.32 & 12.5 \\
\hline 3631.98 & +314939.6 & 18.37 & 16.94 & 17.29 & 14.44 & 13.16 & 12.44 & 4.3 \\
\hline 053706.57 & +274724.2 & 19.40 & 17.85 & 18.04 & 15.41 & 13.87 & 12.81 & 6.5 \\
\hline 053712.44 & +274804.0 & 16.42 & 15.45 & 15.23 & 13.43 & 12.38 & 11.66 & 6.7 \\
\hline 053713.51 & +265659.2 & 18.66 & 17.47 & 17.65 & 15.88 & 14.75 & 13.70 & 16.2 \\
\hline 3728.60 & +320001.5 & 17.70 & 16.70 & 16.32 & 14.99 & 13.82 & 13.00 & 13.0 \\
\hline 3751.20 & +270346.0 & 18.40 & 17.38 & 16.98 & 15.20 & 14.18 & 3.49 & 12.0 \\
\hline 053817.88 & +313934.8 & 16.23 & 15.19 & 14.88 & 13.46 & 12.63 & 11.71 & 22.5 \\
\hline 053821.92 & +272832.4 & 18.24 & 17.14 & 17.09 & 15.00 & 14.03 & 13.33 & 11.0 \\
\hline 053823.09 & +230218.3 & 17.38 & 16.51 & 16.22 & 14.13 & 13.05 & 12.29 & 42.9 \\
\hline 06.05 & +304933.0 & 17.92 & 16.90 & & 5.22 & 36 & .72 & 18.9 \\
\hline 053917.83 & +272747.0 & 18.50 & 17.26 & 17.00 & 14.80 & 13.73 & 13.04 & 11.7 \\
\hline 053923.87 & +272650.4 & 18.94 & 17.64 & 17.43 & 15.43 & 14.15 & 13.26 & 12.8 \\
\hline 053927.29 & +305151.0 & 19.39 & 17.99 & 17.53 & 15.47 & 14.39 & 13.70 & 18.2 \\
\hline 3955.76 & +332412.8 & 16.30 & 15.27 & 15.32 & 13.20 & 12.00 & 11.00 & 46.8 \\
\hline 0.97 & +352724.4 & 18.60 & 17.19 & 17.21 & .72 & .35 & 2.47 & 20.1 \\
\hline 054054.32 & +352554.9 & 16.40 & 15.59 & 15.03 & 14.05 & 12.94 & 12.18 & 21.1 \\
\hline 054132.75 & +265513.6 & 17.33 & 15.69 & 16.11 & 38 & 12.47 & 12.00 & 35.7 \\
\hline 4146.63 & +272748.0 & 18.37 & 17.17 & 16.75 & 14.99 & 14.03 & 13.46 & 22.8 \\
\hline .34 & +341200.6 & 17.88 & 16.67 & 16.93 & 14.55 & 3.52 & 12.87 & 41.3 \\
\hline 054240.94 & +334515.2 & 18.91 & 17.90 & 17.47 & 15.98 & 15.03 & 14.27 & 34.8 \\
\hline 054252.04 & +310014.2 & 18.19 & 17.07 & 17.13 & 90 & 13.91 & 13.28 & 27.5 \\
\hline 054424.19 & +312031.4 & 16.36 & 15.21 & 15.38 & 13.33 & 12.24 & 11.61 & 38.8 \\
\hline 054621.54 & +262724.0 & 15.60 & 14.70 & 14.31 & 12.68 & 11.68 & 10.95 & 24.1 \\
\hline 054635.52 & +261909.8 & 17.68 & 16.63 & 16.73 & 14.61 & 60 & 12.96 & 26.4 \\
\hline 054703.77 & +210034.8 & 18.11 & 16.54 & 16.75 & 11.56 & 10.74 & 10.28 & 40.2 \\
\hline 054713.95 & +221100.5 & 18.88 & 18.09 & 17.81 & 16.45 & 15.56 & 15.09 & 29.4 \\
\hline 4745.79 & +224829.1 & 18.61 & 17.08 & 17.00 & 14.13 & 12.97 & 12.18 & 23.2 \\
\hline 4753.19 & +303 & 18.53 & 17.44 & 16.93 & 15.31 & & 13.76 & 32.1 \\
\hline 054807.74 & +225653.6 & 18.65 & 17.12 & 17.40 & 14.69 & 13.39 & 12.66 & 24.8 \\
\hline 054903.72 & +222708.7 & 18.10 & 17.05 & 16.91 & 14.87 & 13.63 & 12.90 & 29.6 \\
\hline 054912.30 & +330724.5 & 17.99 & 16.99 & 16.91 & 14.50 & 13.19 & 12.16 & 17.6 \\
\hline 05491 & +270021.7 & 19.20 & 17.37 & 17.84 & 13.81 & 12.55 & 11.59 & 13.3 \\
\hline 05493 & +231931.7 & 18.08 & 16.90 & 16.48 & 14.80 & 13.73 & 13.14 & 38.0 \\
\hline 054942.86 & +282315.6 & 18.42 & 17.26 & 17.25 & 37 & 38 & .68 & 18.2 \\
\hline 054945.05 & +270416.1 & 16.97 & 15.90 & 15.79 & 13.67 & 12.60 & 11.83 & 8.7 \\
\hline 054950.54 & +270701.5 & 16.42 & 15.38 & 15.48 & 13.90 & 13.00 & 12.36 & 8.6 \\
\hline 055028.14 & +235951.1 & 19.45 & 18.20 & 18.34 & 15.86 & 14.75 & 14.12 & 21.7 \\
\hline 055031.16 & +312746.0 & 17.98 & 17.17 & 16.98 & 15.57 & 14.61 & 13.99 & 31.9 \\
\hline 055038.56 & +270315.8 & 19.12 & 17.47 & 17.80 & 14.76 & 13.24 & 12.27 & 15.4 \\
\hline 055050.63 & +240611.9 & 17.65 & 16.76 & 16.59 & 14.65 & 13.60 & 12.85 & 19.7 \\
\hline 055112.18 & +254603.3 & 18.51 & 16.95 & 17.05 & 14.17 & 12.77 & 11.75 & 29.5 \\
\hline 055116.76 & +254213.9 & 17.99 & 16.64 & 16.66 & 13.78 & 12.45 & 11.51 & 29.4 \\
\hline 055201.57 & +265733.7 & 19.12 & 17.76 & 17.91 & 15.23 & 14.00 & 13.32 & 27.6 \\
\hline 055245.11 & +171119.1 & 16.40 & 15.27 & 15.16 & 13.78 & 12.95 & 12.51 & 51.8 \\
\hline 055254.08 & +171424.7 & 16.47 & 15.54 & 14.58 & 13.86 & 13.03 & 12.50 & 51.9 \\
\hline 055532.35 & +274451.4 & 17.25 & 16.31 & 15.89 & 14.77 & 13.86 & 13.31 & 44.6 \\
\hline 055724.37 & +200800.5 & 19.07 & 17.79 & 18.08 & 15.68 & 14.66 & 14.07 & 14.1 \\
\hline 055832.17 & +195712.6 & 19.09 & 17.65 & 17.88 & 15.49 & 14.53 & 13.99 & 16.2 \\
\hline 055920.12 & +243307.9 & 17.46 & 16.56 & 16.19 & 15.20 & 14.12 & 13.25 & 34.8 \\
\hline 055923.17 & +165633.5 & 19.41 & 18.19 & 18.26 & 15.84 & 14.87 & 14.02 & 36.0 \\
\hline
\end{tabular}


Table 1. continued.

\begin{tabular}{|c|c|c|c|c|c|c|c|c|}
\hline \multicolumn{2}{|c|}{ RA $(J 2000.0)$ Dec } & $r$ & $i$ & $\mathrm{H} \alpha$ & $\bar{J}$ & $\bar{H}$ & $\overline{K_{\mathrm{s}}}$ & $d_{4}$ \\
\hline 060004.54 & +165126.0 & 18.98 & 18.18 & 17.42 & 16.69 & 15.69 & 14.71 & 28.8 \\
\hline 060019.28 & +201159.2 & 18.16 & 17.09 & 16.66 & 15.26 & 14.29 & 13.73 & 13.8 \\
\hline .17 & +312955.8 & 17.72 & 16.34 & 16.59 & 14.50 & 13.04 & 12.15 & 29.3 \\
\hline 060211.11 & +262232.1 & 18.66 & 18.16 & 17.89 & 16.76 & 15.54 & 14.22 & 50.3 \\
\hline 060236.34 & +30 5744.2 & 19.13 & 18.14 & 17.88 & 16.38 & 15.61 & 15.20 & 23.4 \\
\hline 060240.22 & +312140.2 & 19.44 & 18.51 & 18.49 & 16.90 & 15.95 & 15.34 & 21.2 \\
\hline 060515.15 & +204036.7 & 17.82 & 16.48 & 16.43 & 14.77 & 13.21 & 11.22 & 56.1 \\
\hline 060624.06 & +233235.1 & 17.77 & 16.69 & 16.61 & 14.70 & 13.49 & 12.65 & 64.4 \\
\hline 060633.01 & +300640.5 & 18.28 & 17.24 & 17.05 & 15.75 & 14.58 & 13.92 & 34.7 \\
\hline 060644.63 & +195038.6 & 17.94 & 16.91 & 16.79 & 15.19 & 14.21 & 13.65 & 40.7 \\
\hline 060715.86 & +193000.1 & 15.05 & 14.20 & 14.17 & 13.45 & 12.37 & 11.64 & 32.9 \\
\hline 30.13 & +162739.6 & 15.92 & 15.13 & 15.04 & 13.61 & 12.59 & 11.96 & 45.6 \\
\hline 060852.63 & +203728.3 & 17.14 & 16.25 & 16.25 & 14.42 & 13.51 & 13.04 & 24.3 \\
\hline 060923.62 & +210235.8 & 17.74 & 16.29 & 16.60 & 13.17 & 11.84 & 10.91 & 30.2 \\
\hline 060926.79 & +245519.9 & 16.48 & 15.93 & 15.42 & 12.26 & 11.20 & 10.24 & 39.3 \\
\hline 9.56 & +204126.3 & 18.80 & 17.67 & 17.34 & 15.59 & 14.76 & 14.27 & 24.4 \\
\hline 5.23 & +124022.8 & 17.97 & 16.66 & 16.95 & 13.97 & 12.41 & 11.10 & 15.6 \\
\hline 061008.00 & +121734.8 & 18.88 & 17.75 & 17.42 & 15.35 & 14.03 & 13.08 & 22.7 \\
\hline 061024.41 & +124647.6 & 15.88 & 15.05 & 14.22 & 13.30 & 12.28 & 11.51 & 18.1 \\
\hline 1045.52 & +194817.0 & 17.83 & 16.61 & 16.73 & 15.05 & 13.89 & 13.21 & 31.9 \\
\hline .24 & 352.6 & 19.26 & 17.93 & 17. & 12 & 85 & 2.91 & 33.5 \\
\hline 061258.84 & +15 1835.4 & 18.12 & 16.94 & 16.95 & 15.05 & 13.80 & 12.93 & 5.0 \\
\hline 061302.76 & +153527.3 & 17.80 & 16.66 & 16.70 & 14.68 & 13.69 & 13.12 & 14.6 \\
\hline 061312.70 & +152036.7 & 14.79 & 14.01 & 13.87 & 12.44 & 11.44 & 10.57 & 4.2 \\
\hline 1314.30 & +175343.9 & 17.76 & 16.79 & 16.61 & 14.95 & 13.95 & 13.34 & 27.4 \\
\hline .53 & +15 & 19.48 & 18.34 & 18.12 & 6.07 & 29 & 14.29 & 4.6 \\
\hline 061322.66 & +215746.3 & 18.13 & 17.26 & 17.23 & 15.11 & 14.17 & 13.52 & 28.0 \\
\hline 061328.57 & +160556.9 & 16.10 & 14.95 & 14.99 & 13.11 & 12.05 & 11.47 & 29.2 \\
\hline 1417.27 & +225418.6 & 18.60 & 18.11 & 16.70 & 14.55 & 13.29 & 12.28 & 22.4 \\
\hline 29.10 & +200956.5 & 18.49 & 17.26 & 17.32 & 15.61 & .43 & 3.68 & 18.1 \\
\hline 061522.28 & +1856 55.2 & 18.66 & 17.64 & 17.55 & 15.60 & 14.76 & 14.07 & 30.2 \\
\hline 061638.92 & +200151.6 & 19.37 & 18.14 & 17.99 & 15.80 & 14.23 & 13.25 & 29.9 \\
\hline 061719.51 & +233606.8 & 17.50 & 16.34 & 16.35 & 14.12 & 12.72 & 11.78 & 34.9 \\
\hline 9.67 & +245125.0 & 18.05 & 17.01 & 16.71 & 15.42 & 14.52 & 13.73 & 43.3 \\
\hline 061729.17 & +222152.4 & 17.26 & 16.36 & 16.32 & 14.85 & 13.85 & 13.31 & 30.6 \\
\hline 061758.15 & +241149.2 & 19.16 & 18.12 & 17.90 & 16.27 & 15.32 & 14.47 & 34.0 \\
\hline 061804.17 & +183010.3 & 18.50 & 17.34 & 17.15 & 14.96 & 14.11 & 13.50 & 38.8 \\
\hline 05.61 & +174043.0 & 19.12 & 18.01 & 17.76 & 15.96 & 14.89 & 14.10 & 36.1 \\
\hline 06.94 & +130203.7 & 17.82 & 16.87 & 16.26 & 15.39 & 14.41 & 13.80 & 57.7 \\
\hline 062021.71 & +110710.2 & 18.62 & 17.45 & 17.44 & 16.22 & 15.07 & 13.99 & 40.1 \\
\hline 062047.11 & +105725.7 & 19.17 & 18.12 & 17.65 & 16.26 & 15.04 & 14.15 & 39.5 \\
\hline 062206.60 & +223429.5 & 17.99 & 16.83 & 16.65 & 15.08 & 13.80 & 12.94 & 10.2 \\
\hline 2311.57 & +234251.3 & 18.85 & 17.59 & 17.63 & 15.36 & 14.28 & 13.37 & 30.6 \\
\hline 062335.18 & +231041.0 & 19.35 & 18.05 & 17.92 & 14.86 & 13.76 & 13.02 & 15.2 \\
\hline 062341.27 & +225805.9 & 18.71 & 17.41 & 17.62 & 15.08 & 14.12 & 13.42 & 11.3 \\
\hline 062352.13 & +142619.2 & 17.36 & 16.35 & 16.15 & 14.60 & 13.59 & 12.92 & 13.1 \\
\hline 062421.99 & +143315.3 & 18.31 & 17.17 & 17.29 & 15.16 & 14.14 & 13.45 & 12.1 \\
\hline 062438.78 & +09 1825.9 & 18.14 & 17.15 & 16.72 & 15.34 & 14.53 & 13.79 & 10.6 \\
\hline 062842.43 & +093209.2 & 15.26 & 14.22 & 14.28 & 12.68 & 11.84 & 11.35 & 36.6 \\
\hline 063037.20 & +045402.7 & 16.70 & 15.51 & 15.59 & 12.93 & 11.66 & 10.71 & 14.0 \\
\hline 063039.33 & +052142.1 & 17.11 & 15.89 & 15.96 & 13.70 & 12.66 & 12.08 & 20.3 \\
\hline 063048.17 & +094604.2 & 16.62 & 15.45 & 15.04 & 13.53 & 12.62 & 12.09 & 15.9 \\
\hline 063056.81 & +043834.1 & 16.82 & 15.83 & 15.65 & 13.83 & 12.80 & 12.08 & 15.3 \\
\hline 063101.43 & +104102.0 & 18.30 & 16.46 & 17.11 & 13.74 & 12.59 & 11.95 & 14.2 \\
\hline 063102.58 & +095920.6 & 17.82 & 16.45 & 16.34 & 15.66 & 14.22 & 13.43 & 7.9 \\
\hline & +100126.3 & 18.68 & 17.14 & 17.38 & 14.88 & 14.14 & 13.73 & 8.2 \\
\hline 063109.62 & +102557.5 & 17.35 & 15.43 & 15.73 & 12.85 & 11.88 & 11.27 & 1.7 \\
\hline 063110.28 & +095917.2 & 14.44 & 13.51 & 13.51 & 11.60 & 10.76 & 10.25 & 7.8 \\
\hline 063113.16 & +102657.2 & 19.38 & 16.94 & 17.97 & 14.32 & 13.63 & 13.21 & 1.3 \\
\hline & +102700.0 & 16.87 & 15.31 & 15.14 & 12.93 & 11.90 & 11.25 & 1.3 \\
\hline 063116.26 & +050651.1 & 17.18 & 16.26 & 16.25 & 14.67 & 13.82 & 13.37 & 9.3 \\
\hline
\end{tabular}


R. L. M. Corradi et al.: IPHAS and the symbiotic stars. I., Online Material p 12

Table 1. continued.

\begin{tabular}{|c|c|c|c|c|c|c|c|c|}
\hline \multicolumn{2}{|c|}{ RA (J2000.0) Dec } & $r$ & $i$ & $\mathrm{H} \alpha$ & $J$ & $\bar{H}$ & $\overline{K_{\mathrm{s}}}$ & $d_{4}$ \\
\hline 063119.11 & +102246.5 & 19.10 & 17.59 & 17.81 & 14.37 & 12.81 & 11.91 & 3.5 \\
\hline 063119.66 & +045241.9 & 18.06 & 16.91 & 16.77 & 15.09 & 14.13 & 13.58 & 6.0 \\
\hline 127.85 & +045002.9 & 16.44 & 15.62 & 15.26 & 13.79 & 12.80 & 12.01 & 4.8 \\
\hline 063129.67 & +102323.1 & 17.18 & 15.86 & 15.78 & 13.39 & 12.32 & 11.74 & 3.2 \\
\hline 063130.96 & +0506 58.5 & 15.17 & 14.37 & 14.21 & 12.88 & 12.08 & 11.60 & 7.4 \\
\hline 063146.86 & +100748.5 & 17.09 & 15.40 & 15.96 & 13.54 & 12.69 & 12.26 & 7.3 \\
\hline 3147.05 & +044811.0 & 18.30 & 17.17 & 17.07 & 15.24 & 14.36 & 13.81 & 4.4 \\
\hline 063148.05 & +045048.3 & 17.58 & 16.28 & 16.48 & 14.15 & 13.25 & 12.76 & 3.0 \\
\hline 063206.21 & +103627.7 & 14.80 & 13.30 & 13.73 & 11.01 & 10.22 & 9.61 & 12.8 \\
\hline 063214.59 & +100737.1 & 17.50 & 16.33 & 16.03 & 13.82 & 12.86 & 12.35 & 6.3 \\
\hline 3221.34 & +044619.2 & 17.41 & 16.29 & 16.44 & 14.27 & 13.34 & 12.79 & 9.0 \\
\hline 3231.50 & +045453.4 & 18.83 & 17.74 & 16.71 & 15.80 & 14.75 & 14.12 & 7.8 \\
\hline 063243.35 & +050220.6 & 17.34 & 16.49 & 16.02 & 14.77 & 13.90 & 13.29 & 10.9 \\
\hline 063246.98 & +101638.1 & 17.78 & 16.49 & 16.12 & 12.77 & 11.46 & 10.62 & 7.1 \\
\hline 063322.32 & +043638.2 & 16.70 & 15.76 & 15.56 & 14.09 & 13.10 & 12.49 & 19.7 \\
\hline 32.26 & +045739.5 & 18.24 & 17.04 & 16.73 & 15.12 & 13.78 & 12.73 & 12.4 \\
\hline 32.39 & +045749.2 & 16.32 & 15.34 & 15.05 & 13.57 & 12.56 & 11.87 & 12.4 \\
\hline 063416.90 & +04 2419.2 & 17.13 & 15.93 & 15.97 & 13.78 & 12.75 & 12.05 & 31.3 \\
\hline 063625.88 & +021616.2 & 16.93 & 15.79 & 15.74 & 13.91 & 12.65 & 11.83 & 10.1 \\
\hline 3642.85 & +053736.8 & 17.70 & 16.78 & 16.39 & 14.85 & 14.01 & 13.35 & 24.2 \\
\hline 4.07 & 046.8 & 18.18 & 16.86 & 17. & & .83 & 3.27 & 37.5 \\
\hline 063849.77 & +06 2335.4 & 17.85 & 16.95 & 16.91 & 15.35 & 14.26 & 13.54 & 19.6 \\
\hline 063934.25 & +062116.9 & 18.80 & 18.31 & 17.48 & 16.77 & 15.79 & 14.79 & 13.0 \\
\hline 064222.19 & -022628.6 & 19.22 & 17.77 & 17.94 & 15.85 & 14.90 & 13.75 & 26.2 \\
\hline 850.75 & -014043.3 & 18.93 & 17.64 & 17.77 & 15.67 & 14.59 & 13.82 & 64.5 \\
\hline 5.04 & -00( & 18.35 & 16.26 & 17.14 & 3.69 & 12.14 & 11.09 & 48.8 \\
\hline 182850.22 & +000949.6 & 17.86 & 15.78 & 16.43 & 13.01 & 11.33 & 10.30 & 48.2 \\
\hline 183753.26 & +00 1849.2 & 19.06 & 16.48 & 17.52 & 13.05 & 11.84 & 11.16 & 37.6 \\
\hline 814.63 & -012213.8 & 17.30 & 15.30 & 15.75 & 12.16 & 10.98 & 10.10 & 47.0 \\
\hline 8.54 & 716.5 & 19.27 & 17.40 & 8.14 & .57 & .35 & 2.64 & 43.7 \\
\hline 184222.67 & +025807.0 & 16.03 & 15.04 & 15.08 & 13.32 & 12.36 & 11.80 & 46.7 \\
\hline 184431.46 & -001652.4 & 17.21 & 15.76 & 16.00 & 12.54 & 10.87 & 9.56 & 42.1 \\
\hline 184627.71 & +002817.8 & 18.94 & 17.23 & 17.57 & 14.06 & 12.79 & 12.02 & 26.0 \\
\hline 635.85 & +005 & 14.86 & 13.69 & 13.70 & 11.73 & 10.04 & 8.72 & 37.4 \\
\hline 185005.71 & -004041.2 & 16.95 & 15.59 & 15.72 & 13.00 & 11.87 & 10.65 & 36.1 \\
\hline 185138.28 & -021426.0 & 15.14 & 13.54 & 13.88 & 10.62 & 9.15 & 8.02 & 38.2 \\
\hline 185156.32 & +06 1946.1 & 17.81 & 16.00 & 16.28 & 13.27 & 12.17 & 11.55 & 44.3 \\
\hline 1.44 & +021525.3 & 18.91 & 16.78 & 17.45 & 13.78 & 12.34 & 11.53 & 40.0 \\
\hline .55 & +05 & 18.48 & 17.08 & 16.70 & 14.34 & 13.06 & 12.26 & 51.4 \\
\hline 185403.70 & +035526.7 & 19.30 & 17.62 & 18.05 & 14.28 & 12.71 & 11.63 & 57.9 \\
\hline 185424.82 & +04 1905.0 & 18.29 & 16.46 & 17.10 & 13 & 11.92 & 11.20 & 59.8 \\
\hline 185506.04 & +005426.9 & 19.02 & 17.15 & 17.63 & 15.36 & 13.54 & 12.23 & 25.3 \\
\hline 185526.72 & -040537.5 & 19.30 & 16.76 & 18.00 & 14.23 & 13.51 & 13.12 & 17.2 \\
\hline 185707.14 & +022226.0 & 18.79 & 17.19 & 17.43 & 14.30 & 12.97 & 12.08 & 66.1 \\
\hline 190015.86 & +00 0517.3 & 14.94 & 13. & 13.99 & 12.18 & 11.30 & 10.30 & 32.6 \\
\hline 190021.58 & +052001.1 & 17.90 & 16.73 & 15.95 & 13 & 12.09 & 10.83 & 56.8 \\
\hline 190229.97 & -022757.0 & 16.13 & 16.30 & 13.19 & 13.42 & 11.22 & 9.34 & 12.8 \\
\hline 190346.84 & +162616.9 & 13.98 & 11.95 & 12.08 & 8.73 & 7.92 & 7.50 & 49.8 \\
\hline 190857.31 & +053620.6 & 16.89 & 15.82 & 15.95 & 12.83 & 11.47 & 10.35 & 42.1 \\
\hline 191017.43 & +065258.1 & 15.81 & 13.89 & 14.10 & 10.42 & 8.71 & 7.22 & 84.3 \\
\hline 191233.23 & +114631.2 & 18.60 & 16.86 & 17.09 & 13.99 & 12.67 & 10.92 & 33.2 \\
\hline 191701.33 & +155947.8 & 17.70 & 16.20 & 16.34 & 13.43 & 12.06 & 10.76 & 27.0 \\
\hline 191930.75 & +133242.5 & 19.48 & 18.05 & 18.29 & 15.91 & 15.15 & 14.65 & 60.5 \\
\hline 192033.79 & +231040.3 & 16.89 & 15.39 & 15.85 & 13.43 & 12.65 & 12.22 & 56.9 \\
\hline 192249.80 & +142236.3 & 16.96 & 15.32 & 15.62 & 12.61 & 11.19 & 9.88 & 66.5 \\
\hline 192257.72 & +113854.8 & 18.63 & 17.28 & 17.50 & 14.28 & 12.50 & 11.07 & 59.8 \\
\hline 192400.05 & +230253.1 & 15.79 & 14.68 & 14.56 & 12.82 & 11.97 & 11.34 & 22.1 \\
\hline 192504.10 & +224438.5 & 19.04 & 17.17 & 17.67 & 14.82 & 14.09 & 13.66 & 2.8 \\
\hline 192514.31 & +224144.1 & 16.84 & 15.39 & 15.69 & 13.33 & 12.43 & 11.91 & 3.5 \\
\hline 192517.16 & +223355.9 & 16.14 & 14.69 & 14.88 & 12.77 & 11.83 & 11.33 & 7.4 \\
\hline 192538.41 & +223414.6 & 14.68 & 13.70 & 13.65 & 11.79 & 10.54 & 9.61 & 7.6 \\
\hline
\end{tabular}


Table 1. continued.

\begin{tabular}{|c|c|c|c|c|c|c|c|c|}
\hline \multicolumn{2}{|c|}{ RA $(J 2000.0)$ Dec } & $r$ & $i$ & $\mathrm{H} \alpha$ & $\bar{J}$ & $\bar{H}$ & $\overline{K_{\mathrm{s}}}$ & $d_{4}$ \\
\hline 192547.52 & +222651.3 & 17.56 & 16.56 & 15.71 & 14.71 & 13.92 & 13.23 & 12.5 \\
\hline 192619.04 & +224546.6 & 17.36 & 15.77 & 16.28 & 13.52 & 12.52 & 11.90 & 10.6 \\
\hline 192639.93 & +210705.3 & 14.66 & 13.82 & 13.79 & 12.32 & 11.47 & 10.95 & 8.1 \\
\hline 192701.88 & +224542.0 & 17.02 & 15.49 & 15.98 & 12.38 & 10.97 & 10.04 & 6.7 \\
\hline 192708.06 & +224622.5 & 16.12 & 14.60 & 14.93 & 11.90 & 10.68 & 9.90 & 7.5 \\
\hline 192717.94 & +08 1429.4 & 17.20 & 17.03 & 16.28 & 15.32 & 13.99 & 12.76 & 44.4 \\
\hline 192802.95 & +171643.3 & 19.25 & 17.57 & 17.53 & 14.17 & 12.57 & 10.88 & 35.5 \\
\hline 192819.52 & +215537.9 & 18.73 & 16.88 & 17.56 & 14.74 & 13.82 & 13.25 & 16.3 \\
\hline 192841.27 & +1748 19.9 & 16.70 & 15.49 & 15.47 & 13.67 & 12.43 & 11.48 & 21.3 \\
\hline 192913.09 & +175923.4 & 16.91 & 15.70 & 15.87 & 13.78 & 12.73 & 12.06 & 14.0 \\
\hline 192954.41 & +181026.0 & 17.68 & 16.15 & 16.21 & 13.54 & 12.16 & 11.20 & 10.4 \\
\hline 193000.51 & +182123.0 & 18.96 & 17.20 & 17.74 & 14.87 & 13.36 & 12.49 & 7.6 \\
\hline 193012.69 & +174836.5 & 18.74 & 17.27 & 17.18 & 14.37 & 13.02 & 12.07 & 17.3 \\
\hline 193024.47 & +181938.3 & 19.27 & 17.51 & 17.90 & 13.80 & 12.16 & 10.96 & 7.2 \\
\hline 193038.84 & +183909.8 & 13.26 & 11.35 & 11.95 & 8.21 & 7.28 & 6.60 & 15.0 \\
\hline 19305 & +222228.3 & 17.16 & 15.45 & 15.97 & 12.72 & 11.94 & 11.36 & 36.3 \\
\hline 19310 & +164950.5 & 19.34 & 16.97 & 17.80 & 13.20 & 11.77 & 10.70 & 19.5 \\
\hline 193232.88 & +151711.5 & 14.16 & 13.27 & 13.24 & 11.73 & 10.98 & 10.20 & 79.8 \\
\hline 193311.62 & +175659.0 & 17.94 & 16.69 & 16.87 & 14.61 & 13.51 & 12.77 & 21.6 \\
\hline 193431.49 & +215539.9 & 17.79 & 16.20 & 16.59 & 13.79 & 12.80 & 12.21 & 45.3 \\
\hline 19343 & +182 & 17.23 & 15.87 & 16.22 & 13.38 & 2.20 & 1.41 & 34.1 \\
\hline 193709.65 & +202655.7 & 18.75 & 17.30 & 17.59 & 14.93 & 13.68 & 12.48 & 22.8 \\
\hline 193751.66 & +244408.0 & 19.32 & 17.72 & 18.08 & 15.40 & 14.55 & 14.01 & 19.7 \\
\hline 193852.27 & +203848.8 & 19.43 & 17.98 & 18.00 & 15.51 & 14.43 & 13.75 & 19.5 \\
\hline 194130.75 & +215650.2 & 18.34 & 16.73 & 16.90 & 14.04 & 13.08 & 12.47 & 23.0 \\
\hline 19420 & +212857.8 & 17.89 & 16.02 & 16.62 & 12.95 & 12.04 & 1.38 & 24.4 \\
\hline 194238.90 & +225324.1 & 18.86 & 17.07 & 17.50 & 14.29 & 12.81 & 11.79 & 13.5 \\
\hline 194254.98 & +232414.9 & 13.53 & 12.30 & 12.55 & 10.51 & 9.56 & 8.62 & 3.4 \\
\hline 19425 & +232427.7 & 18.55 & 17.30 & 17.48 & 15.24 & 14.43 & 13.86 & 3.1 \\
\hline 194325.03 & +231347.6 & 19.26 & 17.74 & 18.16 & 5.34 & .08 & 3.36 & 4.4 \\
\hline 194344.62 & +231444.7 & 18.55 & 17.20 & 17.52 & 14.75 & 13.36 & 12.28 & 5.0 \\
\hline 194405.25 & +232647.9 & 14.05 & 13.54 & 11.78 & 11.77 & 10.30 & 9.08 & 8.7 \\
\hline 194423.55 & +235946.4 & 19.17 & 17.50 & 18.08 & 14.76 & 13.72 & 13.11 & 8.3 \\
\hline 194429.90 & +233531.4 & 18.10 & 16.93 & 16.94 & 14.76 & 13.65 & 12.99 & 6.0 \\
\hline 194433.20 & +234430.2 & 18.82 & 17.60 & 17.77 & 15.63 & 14.37 & 13.65 & 4.3 \\
\hline 194442.55 & +272248.6 & 19.39 & 17.94 & 18.25 & 15.74 & 15.02 & 14.52 & 14.9 \\
\hline 194445.16 & +234439.5 & 19.33 & 17.94 & 18.19 & 15.75 & 14.33 & 13.36 & 2.5 \\
\hline 194504.72 & +263950.6 & 18.48 & 16.93 & 17.43 & 14.50 & 13.68 & 13.03 & 29.4 \\
\hline 194513.64 & +234 & 19.18 & 17.64 & 17.95 & 14.76 & 13.57 & 12.92 & 5.5 \\
\hline 194513.87 & +275045.6 & 19.24 & 18.03 & 18.28 & 15.34 & 14.17 & 13.35 & 12.5 \\
\hline 194607.52 & +223112.3 & 17.54 & 16.04 & 14.70 & 9.22 & 7.19 & 5.97 & 33.2 \\
\hline 194609.56 & +225423.2 & 15.20 & 13.69 & 14.12 & 11.50 & 10.79 & 10.24 & 19.2 \\
\hline 194907.23 & +211742.0 & 16.77 & 15.99 & 15.05 & 13.58 & 11.88 & 10.49 & 39.2 \\
\hline 19492 & +285809.4 & 17.13 & 15.86 & 16.03 & 13.78 & 12.97 & 12.23 & 13.6 \\
\hline 194948.25 & +235331.8 & 16.03 & 14.94 & 14.94 & 03 & 11.97 & 11.25 & 33.9 \\
\hline 195001.48 & +262738.1 & 16. & 15. & 15.41 & 13 & 11.74 & 10.62 & 28.2 \\
\hline 195444.83 & +335153.9 & 18. & 17.34 & 17.67 & 15.27 & 14.20 & 13.54 & 30.2 \\
\hline 19561 & +301528.6 & 18.35 & 17.42 & 17.45 & 15.22 & 14.25 & 13.43 & 4.7 \\
\hline 195655.88 & +315637.0 & 16.39 & 15.30 & 14.95 & 13.04 & 12.19 & 11.58 & 7.5 \\
\hline 195712.42 & +301316.1 & 13.02 & 12.22 & 12.10 & 10.30 & 9.31 & 8.10 & 7.9 \\
\hline 195934.39 & +320848.7 & 16.03 & 15.00 & 15.03 & 12.81 & 12.07 & 11.53 & 14.2 \\
\hline 195935.55 & +283830.3 & 15.81 & 14.95 & 14.58 & 12.48 & 10.32 & 8.27 & 20.1 \\
\hline 195947.22 & +353052.9 & 17.05 & 16.25 & 16.09 & 14.80 & 14.01 & 13.04 & 32.9 \\
\hline 195956.42 & +304823.8 & 17.48 & 16.44 & 15.90 & 13.69 & 12.16 & 10.91 & 7.0 \\
\hline 200112.85 & +282211.3 & 17.72 & 16.55 & 16.40 & 14.27 & 13.05 & 12.20 & 26.5 \\
\hline 200153.90 & +354222.3 & 17.85 & 16.50 & 16.61 & 14.33 & 13.20 & 12.37 & 26.5 \\
\hline 200154.27 & +292848.4 & 18.81 & 17.39 & 17.55 & 15.11 & 14.12 & 13.53 & 32.3 \\
\hline 200159.69 & +320635.4 & 16.13 & 14.62 & 15.10 & 11.61 & 10.75 & 10.08 & 13.1 \\
\hline 200203.12 & +323245.5 & 17.41 & 16.73 & 16.26 & 13.67 & 12.80 & 11.98 & 19.1 \\
\hline 200233.05 & +351538.8 & 18.50 & 17.23 & 17.50 & 14.96 & 14.01 & 13.49 & 5.7 \\
\hline 200423.15 & +354007.1 & 18.10 & 16.60 & 16.97 & 14.32 & 13.25 & 12.56 & 6.5 \\
\hline
\end{tabular}


Table 1. continued.

\begin{tabular}{|c|c|c|c|c|c|c|c|c|}
\hline \multicolumn{2}{|c|}{ RA (J2000.0) Dec } & $r$ & $i$ & $\mathrm{H} \alpha$ & $J$ & $\bar{H}$ & $\overline{K_{\mathrm{s}}}$ & $d_{4}$ \\
\hline 200447.02 & +354709.5 & 18.85 & 17.88 & 17.49 & 16.04 & 15.22 & 14.77 & 8.7 \\
\hline 200500.61 & +353752.2 & 19.34 & 17.75 & 18.06 & 15.67 & 14.76 & 14.26 & 4.9 \\
\hline 200514.59 & +322125.1 & 17.24 & 17.63 & 14.18 & 14.96 & 12.76 & 10.66 & 15.3 \\
\hline 200521.89 & +295156.6 & 17.41 & 16.39 & 16.49 & 13.76 & 12.37 & 11.25 & 35.9 \\
\hline 200651.26 & +272037.6 & 17.98 & 16.39 & 16.40 & 13.47 & 12.09 & 11.01 & 35.4 \\
\hline 200707.58 & +355006.3 & 17.95 & 16.96 & 16.83 & 15.14 & 14.15 & 13.50 & 9.1 \\
\hline 200714.83 & +353601.9 & 19.24 & 18.09 & 18.03 & 16.46 & 15.24 & 14.50 & 3.9 \\
\hline 200726.84 & +353436.0 & 19.39 & 17.95 & 17.69 & 15.99 & 15.10 & 14.57 & 2.9 \\
\hline 200728.65 & +361829.3 & 18.20 & 17.16 & 16.66 & 15.06 & 14.26 & 13.64 & 23.6 \\
\hline 200811.53 & +352500.5 & 15.58 & 15.00 & 14.18 & 11.31 & 10.61 & 9.78 & 8.8 \\
\hline 200911.34 & +3034 11.7 & 18.39 & 17.02 & 17.10 & 13.86 & 12.66 & 11.77 & 36.7 \\
\hline 201022.77 & +2956 21.5 & 17.95 & 16.75 & 16.93 & 15.50 & 14.39 & 13.76 & 31.3 \\
\hline 201024.42 & +314516.6 & 17.82 & 15.97 & 16.36 & 12.87 & 11.81 & 10.90 & 31.0 \\
\hline 201057.51 & +343732.4 & 15.68 & 14.36 & 14.64 & 12.08 & 10.84 & 9.75 & 20.1 \\
\hline 201126.20 & +331606.8 & 18.93 & 17.55 & 17.58 & 14.34 & 12.94 & 11.70 & 19.1 \\
\hline 52.98 & +281517.0 & 17.39 & 15.33 & 15.93 & 12.30 & 10.71 & 9.50 & 35.6 \\
\hline 201231.94 & +322214.0 & 15.27 & 14.56 & 14.42 & 13.13 & 12.18 & 10.82 & 25.8 \\
\hline 201307.52 & +31 1851.5 & 18.36 & 16.82 & 16.95 & 13.96 & 12.63 & 11.59 & 27.1 \\
\hline 201358.71 & +314210.8 & 19.31 & 17.38 & 17.94 & 13.66 & 12.15 & 10.97 & 27.8 \\
\hline 201428.69 & +320909.5 & 18.06 & 16.67 & 16.98 & 14.24 & 13.03 & 12.25 & 14.9 \\
\hline 42.32 & +320903.9 & 17.39 & 16.01 & 16. & 3.38 & 12.11 & 11.26 & 14.4 \\
\hline 201550.96 & +373004.2 & 18.07 & 16.40 & 15.91 & 13.08 & 11.46 & 9.96 & 8.6 \\
\hline 201603.23 & +371450.6 & 19.01 & 17.76 & 17.42 & 15.45 & 14.11 & 13.10 & 7.9 \\
\hline 201622.09 & +381014.2 & 17.40 & 16.31 & 16.06 & 14.84 & 13.91 & 13.35 & 13.8 \\
\hline 1623.18 & +373704.6 & 19.34 & 17.33 & 17.81 & 14.06 & 12.66 & 11.48 & 9.9 \\
\hline 8.91 & +293 & 18.80 & 16.86 & 17.25 & .86 & 12.53 & 11.60 & 68.3 \\
\hline 201704.88 & +404834.2 & 18.36 & 16.95 & 17.35 & 14.85 & 13.78 & 13.20 & 6.6 \\
\hline 201708.12 & +410727.0 & 14.29 & 12.74 & 13.04 & 10.15 & 9.27 & 8.65 & 18.1 \\
\hline 201713.88 & +421044.2 & 16.76 & 15.38 & 15.67 & 13.06 & 11.81 & 10.95 & 31.7 \\
\hline 201715.03 & +310 & 16.00 & 15.13 & 5.08 & 3.54 & 12.58 & 2.06 & 9.8 \\
\hline 201745.30 & +415033.2 & 18.12 & 16.82 & 16.81 & 14.72 & 13.28 & 12.30 & 24.1 \\
\hline 201751.21 & +310052.0 & 18.52 & 17.43 & 16.68 & 14.67 & 13.03 & 11.75 & 13.8 \\
\hline 201835.83 & +405508.0 & 17.24 & 16.22 & 15.61 & 14.75 & 13.81 & 12.88 & 3.7 \\
\hline 67 & +404727.8 & 16.97 & 15.81 & 15.29 & 13.94 & 13.10 & 12.49 & 6.3 \\
\hline 49.29 & +393 & 17.04 & 16.00 & 15.55 & 13.59 & 12.64 & 11.88 & 12.4 \\
\hline 201859.19 & +392442.3 & 17.58 & 16.48 & 16.25 & 76 & 13.68 & 13.02 & 6.8 \\
\hline 201909.01 & +333240.1 & 16.34 & 15.38 & 15.40 & 13.70 & 12.83 & 12.22 & 45.7 \\
\hline 20191 & +360359.3 & 18.27 & 16.61 & 17.16 & 13.16 & 11.28 & 9.71 & 42.6 \\
\hline 20191 & 448.5 & 18.61 & 17.68 & 16.64 & 16.04 & 14.87 & 14.19 & 12.6 \\
\hline 201933.95 & +364110.6 & 17.97 & 16.97 & 16.81 & 10 & .08 & 13.22 & 26.7 \\
\hline 201935.95 & +392443.5 & 19.35 & 17.73 & 17.98 & 15.43 & 14.40 & 13.78 & 5.6 \\
\hline 201945.44 & +394400.9 & 16.36 & 15.17 & 14.45 & 12.98 & 11.83 & 10.97 & 14.6 \\
\hline 52.07 & +324132.3 & 18.29 & 16.86 & 17.15 & 14.18 & 13.04 & 12.25 & 63.6 \\
\hline 07.87 & +400 & 17.96 & 16.85 & 16.48 & 14.85 & 14.11 & 13.55 & 9.2 \\
\hline 202024.83 & +3843 17.5 & 19.18 & 17.77 & 18.07 & 15.17 & 14.05 & 13.44 & 17.1 \\
\hline 202058.52 & +380949.8 & 16.20 & 14.67 & 14.60 & 11.88 & 10.38 & 8.87 & 14.2 \\
\hline 202112.98 & +410113.1 & 18.03 & 16.57 & 16.83 & 14.24 & 13.33 & 12.84 & 14.7 \\
\hline 202210.20 & +335034.6 & 14.38 & 12.90 & 13.34 & 10.58 & 9.84 & 9.28 & 44.6 \\
\hline 202234.45 & +405546.4 & 18.73 & 17.12 & 17.36 & 14.87 & 14.15 & 13.63 & 15.0 \\
\hline 202341.92 & +432538.5 & 18.38 & 17.12 & 17.14 & 14.64 & 13.40 & 12.65 & 33.3 \\
\hline 202345.75 & +390440.2 & 19.25 & 17.58 & 17.95 & 15.32 & 14.44 & 13.87 & 6.9 \\
\hline 202407.29 & +423552.1 & 16.98 & 15.58 & 15.56 & 13.16 & 12.04 & 11.33 & 11.2 \\
\hline 202411.20 & +421709.1 & 16.33 & 15.49 & 15.11 & 13.61 & 12.64 & 11.97 & 6.8 \\
\hline 202421.82 & +422554.3 & 17.43 & 16.28 & 16.09 & 13.59 & 12.23 & 11.25 & 6.5 \\
\hline 202427.02 & +385539.0 & 18.11 & 17.15 & 16.29 & 13.40 & 11.89 & 10.78 & 7.0 \\
\hline 202427.13 & +383054.9 & 17.87 & 16.74 & 16.59 & 14.85 & 13.90 & 13.29 & 3.2 \\
\hline 202429.12 & +390227.1 & 15.07 & 14.17 & 14.01 & 12.42 & 11.42 & 10.66 & 3.2 \\
\hline 202431.08 & +421307.6 & 17.75 & 16.60 & 16.47 & 14.85 & 13.81 & 13.10 & 6.4 \\
\hline 202439.32 & +395655.2 & 19.03 & 17.76 & 17.32 & 15.42 & 14.41 & 13.55 & 33.3 \\
\hline 202439.59 & +422109.0 & 15.90 & 14.77 & 14.73 & 12.65 & 11.54 & 10.75 & 5.5 \\
\hline 202455.53 & +424504.0 & 18.90 & 16.99 & 17.38 & 14.43 & 13.55 & 12.88 & 17.9 \\
\hline
\end{tabular}


Table 1. continued.

\begin{tabular}{|c|c|c|c|c|c|c|c|c|}
\hline \multicolumn{2}{|c|}{ RA $(J 2000.0)$ Dec } & $r$ & $i$ & $\mathrm{H} \alpha$ & $\bar{J}$ & $\bar{H}$ & $\overline{K_{\mathrm{s}}}$ & $d_{4}$ \\
\hline 202530.03 & +344517.5 & 18.37 & 17.04 & 16.88 & 14.94 & 13.60 & 12.70 & 49.0 \\
\hline 202549.86 & +412308.1 & 18.89 & 18.06 & 16.99 & 15.42 & 13.85 & 12.30 & 17.9 \\
\hline 202707.14 & +393248.6 & 16.67 & 15.45 & 15.43 & 12.69 & 11.53 & 10.76 & 17.6 \\
\hline 202709.33 & +39 1421.5 & 17.76 & 16.45 & 16.54 & 13.93 & 12.76 & 12.04 & 13.2 \\
\hline 202742.17 & +403011.1 & 18.21 & 16.86 & 17.09 & 14.05 & 12.94 & 12.26 & 17.9 \\
\hline 202757.89 & +392332.5 & 16.33 & 15.20 & 15.29 & 12.77 & 11.48 & 10.42 & 12.7 \\
\hline 202831.45 & +395320.1 & 14.74 & 13.82 & 13.45 & 11.49 & 10.43 & 9.56 & 18.3 \\
\hline 202834.25 & +355417.4 & 15.89 & 14.42 & 13.81 & 11.50 & 10.08 & 8.29 & 45.6 \\
\hline 202844.46 & +401136.7 & 16.83 & 15.57 & 15.27 & 13.68 & 12.69 & 12.12 & 7.0 \\
\hline 202847.71 & +403349.3 & 16.88 & 15.56 & 15.74 & 13.00 & 11.71 & 10.74 & 14.5 \\
\hline 202850.31 & +403549.8 & 17.84 & 16.25 & 16.70 & 13.66 & 12.09 & 10.91 & 14.9 \\
\hline 202916.22 & +401106.2 & 17.22 & 15.91 & 15.94 & 13.96 & 12.99 & 12.42 & 6.9 \\
\hline 202932.28 & +401307.5 & 15.68 & 14.80 & 14.76 & 13.16 & 12.31 & 11.85 & 8.7 \\
\hline 202947.93 & +355926.5 & 14.75 & 13.74 & 12.98 & 10.96 & 9.44 & 8.14 & 48.1 \\
\hline 203018.58 & +385713.5 & 17.18 & 16.12 & 16.04 & 14.16 & 13.17 & 12.56 & 29.1 \\
\hline .99 & 325.6 & 18.67 & 17.00 & 17.37 & 13.98 & 12.60 & 11.67 & 23.1 \\
\hline 20311 & +402046.5 & 16.64 & 15.45 & 15.24 & 13.24 & 12.04 & 11.21 & 13.7 \\
\hline 203113.75 & +411517.5 & 19.34 & 17.67 & 18.04 & 14.56 & 13.36 & 12.66 & 30.8 \\
\hline 203300.81 & +401800.3 & 17.44 & 16.27 & 16.00 & 14.21 & 13.17 & 12.62 & 22.8 \\
\hline 203301.84 & +390342.7 & 16.73 & 15.31 & 15.33 & 12.78 & 11.54 & 10.53 & 42.6 \\
\hline .45 & +48.9 & 18.77 & 16.92 & 33 & .04 & .70 & 10.86 & 18.4 \\
\hline 203413.39 & +410157.9 & 19.00 & 17.75 & 16.54 & 13.23 & 11.29 & 9.86 & 20.5 \\
\hline 203506.32 & +470322.8 & 16.44 & 15.23 & 15.48 & 12.73 & 11.38 & 10.15 & 32.1 \\
\hline 203526.03 & +464956.1 & 18.54 & 17.17 & 17.53 & 14.72 & 13.66 & 12.95 & 31.5 \\
\hline 4114.48 & +460155.9 & 18.93 & 17.98 & 17.32 & 15.74 & 14.91 & 14.28 & 18.0 \\
\hline 4121.02 & 21.3 & 19.07 & 17.53 & 17.49 & .55 & .87 & 12.74 & 34.7 \\
\hline 204122.99 & +455541.6 & 18.84 & 17.72 & 17.91 & 15.71 & 14.87 & 14.33 & 14.4 \\
\hline 204134.22 & +393239.0 & 15.16 & 14.11 & 13.39 & 12.12 & 10.99 & 10.20 & 15.6 \\
\hline 4140.07 & +411227.9 & 17.35 & 15.74 & 15.99 & 12.82 & 11.43 & 10.46 & 32.7 \\
\hline 02.36 & +430 & 15.29 & 14.13 & 14.08 & 2.51 & .35 & 0.57 & 28.6 \\
\hline 204516.51 & +413526.8 & 17.25 & 15.78 & 15.99 & 13.18 & 12.03 & 11.34 & 24.1 \\
\hline 204529.31 & +410622.3 & 16.61 & 15.47 & 15.59 & 13.38 & 12.05 & 11.08 & 19.8 \\
\hline 204626.87 & +461817.8 & 18.83 & 17.71 & 17.58 & 15.99 & 14.92 & 14.26 & 8.1 \\
\hline 2.98 & +464 & 17.72 & 16.48 & 16.61 & 14.10 & 13.26 & 12.60 & 16.7 \\
\hline 204645.49 & +410659.6 & 18.54 & 17.46 & 17.14 & 15.61 & 14.22 & 13.03 & 21.8 \\
\hline 204704.82 & +434911.4 & 18.76 & 17.53 & 16.71 & 15.34 & 14.34 & 13.76 & 1.6 \\
\hline 204713.69 & +463517.5 & 14.46 & 12.85 & 13.13 & 10.05 & 8.93 & 7.48 & 14.6 \\
\hline 204714.21 & +471 & 17.99 & 17.08 & 16.52 & 15.14 & 14.29 & 13.66 & 16.8 \\
\hline 204725.22 & +434 & 17.82 & 16.57 & 16.10 & 13.35 & 2.21 & 1.35 & 3.4 \\
\hline 204733.97 & +413136.7 & 18.19 & 16.88 & 16.38 & 14.92 & 14.09 & 13.58 & 27.4 \\
\hline 204819.32 & +472047.3 & 16.67 & 15.87 & 15.82 & 14.29 & 13.48 & 12.95 & 14.7 \\
\hline 204857.79 & +434955.1 & 18.33 & 16.84 & 17.27 & 13.60 & 12.26 & 11.40 & 18.7 \\
\hline 4907.37 & 518.0 & 16.46 & 15.49 & 15.07 & 13.72 & 12.93 & 12.53 & 15.3 \\
\hline 204956.23 & +465 & 16.50 & 15.46 & 15.38 & 3.51 & 12.59 & 12.04 & 9.5 \\
\hline 205018.22 & +465356.4 & 19.02 & 17.50 & 17.65 & 29 & 13.96 & 12.88 & 6.3 \\
\hline 205019.55 & +454737.0 & 18.72 & 17.20 & 17.67 & 15.78 & 14.67 & 13.77 & 17.9 \\
\hline 205020.65 & +454721.5 & 17.09 & 15.94 & 16.01 & 13.81 & 12.80 & 12.22 & 18.1 \\
\hline 205022.42 & +441917.6 & 18.94 & 16.97 & 16.79 & 13.91 & 12.73 & 12.09 & 14.5 \\
\hline 205040.29 & +443049.1 & 14.96 & 13.83 & 13.78 & 11.52 & 10.42 & 9.55 & 11.7 \\
\hline 205044.99 & +471244.7 & 18.61 & 17.55 & 17.48 & 15.40 & 14.26 & 13.58 & 6.4 \\
\hline 205050.39 & +445011.7 & 14.80 & 13.44 & 13.69 & 15.41 & 13.10 & 11.10 & 17.4 \\
\hline 205053.17 & +445036.4 & 18.66 & 16.78 & 17.09 & 14.02 & 12.89 & 12.28 & 17.6 \\
\hline 205102.14 & +471237.4 & 18.44 & 16.62 & 17.21 & 13.80 & 12.36 & 11.46 & 6.7 \\
\hline 205120.99 & +442619.6 & 15.25 & 14.10 & 14.09 & 11.88 & 10.96 & 10.30 & 7.3 \\
\hline 205132.79 & +442348.0 & 16.65 & 15.43 & 15.31 & 13.34 & 12.27 & 11.63 & 7.7 \\
\hline 205200.99 & +442841.4 & 16.94 & 15.60 & 15.60 & 13.55 & 12.49 & 11.90 & 7.9 \\
\hline 205240.84 & +495804.1 & 18.48 & 17.00 & 17.45 & 14.18 & 13.34 & 12.68 & 39.9 \\
\hline 205256.75 & +390830.2 & 18.12 & 16.59 & 17.01 & 14.71 & 13.82 & 13.31 & 57.0 \\
\hline 205315.64 & +434422.8 & 17.00 & 15.93 & 15.30 & 14.07 & 13.21 & 12.54 & 29.4 \\
\hline & +445131.9 & 18.28 & 16.56 & 16.96 & 13.90 & 12.80 & 12.20 & 12.3 \\
\hline 205407.40 & +413458.0 & 15.12 & 13.77 & 13.77 & 14.62 & 12.82 & 11.28 & 40.6 \\
\hline
\end{tabular}


Table 1. continued.

\begin{tabular}{|c|c|c|c|c|c|c|c|c|}
\hline \multicolumn{2}{|c|}{ RA (J2000.0) Dec } & $r$ & $i$ & $\mathrm{H} \alpha$ & $J$ & $\bar{H}$ & $\overline{K_{\mathrm{s}}}$ & $d_{4}$ \\
\hline 205433.18 & +484630.8 & 16.47 & 15.34 & 15.39 & 13.39 & 12.68 & 12.10 & 36.8 \\
\hline 205438.00 & +491330.9 & 19.39 & 17.86 & 18.07 & 15.49 & 14.21 & 13.13 & 29.2 \\
\hline 5446.91 & +44 4819.9 & 17.02 & 15.63 & 15.88 & 12.88 & 11.52 & 10.31 & 7.1 \\
\hline 205454.34 & +444343.0 & 17.79 & 16.21 & 16.04 & 14.04 & 13.18 & 12.74 & 6.0 \\
\hline 205458.07 & +4745 51.7 & 14.69 & 13.76 & 13.66 & 12.28 & 11.54 & 10.77 & 20.2 \\
\hline 205503.02 & +441052.1 & 16.41 & 14.95 & 15.15 & 12.43 & 11.11 & 10.25 & 26.3 \\
\hline 205503.99 & +444426.6 & 17.80 & 16.39 & 16.35 & 14.31 & 13.31 & 12.59 & 6.5 \\
\hline 205647.90 & +462434.5 & 17.33 & 15.77 & 16.15 & 13.14 & 12.19 & 11.59 & 13.1 \\
\hline 205720.26 & +42 2319.4 & 17.12 & 15.54 & 15.91 & 12.90 & 11.82 & 11.24 & 51.2 \\
\hline 205730.09 & +493258.0 & 16.21 & 14.87 & 15.16 & 13.15 & 12.46 & 11.84 & 18.6 \\
\hline 205736.62 & +522117.1 & 15.78 & 14.58 & 14.37 & 12.45 & 11.44 & 10.76 & 24.4 \\
\hline 205737.30 & +440644.0 & 17.94 & 16.49 & 16.40 & 13.85 & 12.91 & 12.16 & 16.3 \\
\hline 205740.95 & +465945.1 & 15.79 & 14.43 & 14.62 & 12.26 & 11.57 & 10.99 & 21.9 \\
\hline 205759.87 & +435326.1 & 14.68 & 13.75 & 13.54 & 11.85 & 10.87 & 10.25 & 7.7 \\
\hline 205801.38 & +434520.2 & 16.52 & 15.26 & 15.23 & 13.11 & 11.87 & 11.05 & 9.5 \\
\hline 02.67 & +463502.6 & 19.05 & 17.73 & 17.80 & 15.62 & 14.34 & 13.20 & 14.2 \\
\hline .19 & +462149.0 & 18.31 & 16.73 & 17.21 & 14.24 & 3.33 & 12.72 & 7.6 \\
\hline 205821.54 & +435344.9 & 16.69 & 15.23 & 15.35 & 12.80 & 11.49 & 10.71 & 7.3 \\
\hline 205827.02 & +435320.1 & 17.42 & 16.14 & 16.04 & 14.02 & 12.92 & 12.25 & 7.8 \\
\hline 205842.07 & +462459.8 & 16.42 & 15.13 & 15.17 & 13.04 & 12.32 & 11.74 & 10.4 \\
\hline 6.70 & 823.9 & 17.20 & 16.17 & 16. & 4.22 & .29 & 12.75 & 21.8 \\
\hline 210019.06 & +522728.3 & 14.61 & 13.60 & 13.55 & 11.69 & 10.63 & 9.81 & 5.8 \\
\hline 210021.42 & +522709.6 & 14.06 & 12.88 & 12.84 & 11.15 & 10.22 & 9.40 & 5.6 \\
\hline 210110.58 & +521512.9 & 17.07 & 15.54 & 15.49 & 13.06 & 12.04 & 11.29 & 11.2 \\
\hline 2.56 & +474839.4 & 16.81 & 15.66 & 15.39 & 13.62 & 12.59 & 11.81 & 20.5 \\
\hline .13 & +501 & 18.80 & 16.67 & 17.45 & .85 & .80 & 2.23 & 6.5 \\
\hline 210358.92 & +501451.4 & 15.85 & 14.39 & 14.80 & 12.24 & 11.06 & 10.35 & 4.0 \\
\hline 210404.87 & +535124.4 & 18.21 & 16.63 & 15.81 & 13.43 & 12.40 & 11.61 & 54.6 \\
\hline .83 & +501318.0 & 16.33 & 15.12 & 15.16 & 12.71 & 11.78 & 11.10 & 3.9 \\
\hline 210427.91 & +522203.3 & 16.88 & 15.37 & 15.65 & 2.16 & .10 & 0.42 & 33.6 \\
\hline 210428.81 & +501824.6 & 19.01 & 16.75 & 17.35 & 13.98 & 12.96 & 12.42 & 4.8 \\
\hline 210454.56 & +501751.9 & 16.48 & 15.24 & 14.89 & 13.29 & 12.07 & 11.14 & 7.1 \\
\hline 210528.26 & +513600.8 & 18.09 & 16.07 & 16.66 & 12.91 & 11.84 & 11.06 & 38.0 \\
\hline 21061 & +512141.3 & 18.83 & 17.00 & 17.38 & 14.52 & 13.20 & 12.39 & 35.3 \\
\hline 210613.61 & +502 & 17.60 & 16.34 & 16.42 & 14.24 & 13.37 & 12.80 & 18.4 \\
\hline 210710.22 & +485729.3 & 15.06 & 13.60 & 14.01 & 11.35 & 10.55 & 9.97 & 16.5 \\
\hline 211028.25 & +445841.8 & 16.70 & 15.76 & 15.22 & 14.02 & 13.10 & 12.60 & 34.2 \\
\hline 211053.52 & +511344.0 & 17.06 & 15.62 & 15.99 & 13.35 & 12.63 & 12.05 & 37.1 \\
\hline 211125.36 & +465 & 16.45 & 15.34 & 15.33 & 13.51 & 12.46 & 11.76 & 17.0 \\
\hline 211317.53 & +511430.2 & 17.39 & 15.89 & 16.02 & 13.34 & 12.10 & 11.29 & 29.9 \\
\hline 211318.30 & +462017.9 & 19.33 & 17.98 & 18.33 & 15.04 & 13.91 & 13.24 & 25.1 \\
\hline 211558.05 & +475746.5 & 18.32 & 16.77 & 17.18 & 14.48 & 13.25 & 12.20 & 27.3 \\
\hline 22.51 & +513441.8 & 19.48 & 17.55 & 18.16 & 15.25 & 14.30 & 13.79 & 14.6 \\
\hline 41.03 & +513 & 18.88 & 17.45 & 17.72 & 15.35 & 14.29 & 13.58 & 14.2 \\
\hline 211645.67 & +471536.5 & 17.33 & 16. & 15.82 & 58 & 13.66 & 13.12 & 25.2 \\
\hline 211818.24 & +425352.3 & 17.62 & 16.15 & 16.13 & 13.58 & 12.62 & 11.98 & 24.4 \\
\hline 211934.02 & +464745.5 & 16.54 & 15.39 & 15.38 & 13.12 & 12.19 & 11.69 & 1.7 \\
\hline 211936.96 & +464909.0 & 16.79 & 15.28 & 15.52 & 12.09 & 10.88 & 10.04 & 1.4 \\
\hline 211938.96 & +464913.6 & 18.84 & 17.63 & 17.15 & 15.06 & 13.56 & 12.29 & 1.5 \\
\hline 211947.43 & +532225.1 & 16.13 & 14.64 & 14.94 & 12.08 & 11.16 & 10.56 & 29.4 \\
\hline 211956.24 & +514704.2 & 18.97 & 17.51 & 16.92 & 15.20 & 14.03 & 13.01 & 5.0 \\
\hline 212025.15 & +514630.7 & 18.30 & 16.80 & 17.11 & 14.69 & 13.78 & 13.30 & 5.1 \\
\hline 212036.52 & +515126.0 & 18.55 & 17.39 & 17.40 & 14.87 & 13.53 & 12.51 & 1.3 \\
\hline 212048.00 & +534051.3 & 17.03 & 15.39 & 15.84 & 12.72 & 11.85 & 11.24 & 30.8 \\
\hline 212124.55 & +524955.1 & 17.68 & 16.19 & 16.59 & 13.99 & 13.23 & 12.71 & 29.2 \\
\hline 212145.31 & +465457.9 & 17.45 & 15.94 & 16.24 & 13.79 & 12.69 & 12.07 & 5.0 \\
\hline 212154.68 & +463856.7 & 16.89 & 15.68 & 15.76 & 14.28 & 13.18 & 12.40 & 15.1 \\
\hline 212159.94 & +492624.2 & 18.17 & 16.05 & 16.72 & 13.58 & 12.78 & 12.29 & 16.6 \\
\hline 212200.51 & +465432.7 & 15.69 & 14.25 & 14.54 & 11.21 & 9.79 & 8.59 & 4.6 \\
\hline 212209.02 & +492624.6 & 16.09 & 14.79 & 15.12 & 12.25 & 10.27 & 8.71 & 16.6 \\
\hline 212214.73 & +490538.8 & 19.07 & 17.43 & 17.51 & 14.01 & 12.89 & 12.25 & 19.4 \\
\hline
\end{tabular}


Table 1. continued.

\begin{tabular}{|c|c|c|c|c|c|c|c|c|}
\hline \multicolumn{2}{|c|}{ RA (J2000.0) Dec } & $r$ & $i$ & $\mathrm{H} \alpha$ & $\bar{J}$ & $\bar{H}$ & $\overline{K_{\mathrm{s}}}$ & $d_{4}$ \\
\hline 212218.11 & +465711.2 & 18.20 & 16.17 & 16.23 & 13.55 & 12.76 & 12.32 & 5.9 \\
\hline 212350.19 & +500818.2 & 19.07 & 17.45 & 17.62 & 14.38 & 12.83 & 11.73 & 28.4 \\
\hline 2620.32 & +552551.8 & 16.80 & 15.89 & 15.81 & 13.74 & 12.47 & 11.36 & 11.9 \\
\hline 212955.57 & +553904.1 & 17.81 & 16.52 & 16.15 & 14.19 & 12.74 & 11.38 & 26.7 \\
\hline 213105.96 & +512531.8 & 18.07 & 16.33 & 16.86 & 13.83 & 12.88 & 12.21 & 34.9 \\
\hline 213108.81 & +515945.3 & 19.05 & 17.32 & 17.79 & 14.60 & 13.29 & 12.34 & 41.5 \\
\hline 213125.07 & +491835.5 & 18.04 & 17.50 & 16.36 & 14.85 & 13.71 & 12.88 & 34.7 \\
\hline 213129.48 & +480954.7 & 15.97 & 14.77 & 14.96 & 13.28 & 12.37 & 11.82 & 24.0 \\
\hline 213317.78 & +574813.5 & 14.63 & 13.96 & 13.68 & 12.25 & 10.96 & 9.83 & 12.7 \\
\hline 213431.56 & +541611.8 & 15.41 & 13.94 & 14.31 & 11.97 & 11.21 & 10.63 & 23.3 \\
\hline 213451.70 & +575140.5 & 18.49 & 16.77 & 17.22 & 14.33 & 13.25 & 12.65 & 9.4 \\
\hline 213453.08 & +575125.1 & 18.73 & 17.20 & 17.38 & 14.71 & 13.48 & 12.68 & 9.5 \\
\hline 213516.89 & +573242.3 & 17.93 & 16.50 & 16.16 & 14.44 & 13.56 & 13.07 & 7.4 \\
\hline 213806.68 & +552046.4 & 16.16 & 14.78 & 15.16 & 12.73 & 11.99 & 11.46 & 20.0 \\
\hline 213810.00 & +572352.8 & 18.99 & 17.06 & 17.50 & 14.87 & 14.09 & 13.70 & 10.1 \\
\hline 2.64 & +572033.7 & 17.73 & 16.37 & 16.02 & 14.53 & 13.64 & 13.17 & 10.0 \\
\hline 213929.40 & +570630.7 & 18.13 & 16.54 & 16.96 & 14.50 & 13.72 & 13.33 & 13.6 \\
\hline 213951.88 & +572658.3 & 19.36 & 17.23 & 17.90 & 14.93 & 14.16 & 13.56 & 1.6 \\
\hline 213952.16 & +581412.9 & 17.12 & 15.91 & 15.79 & 13.90 & 12.86 & 12.29 & 4.7 \\
\hline 52.23 & +522515.2 & 19.04 & 17.93 & 18.08 & 15.60 & 14.60 & 13.85 & 44.5 \\
\hline .07 & +572933.5 & 17.76 & 16.36 & 16. & 72 & .87 & .28 & 2.1 \\
\hline 213958.44 & +581214.8 & 17.37 & 15.70 & 16.12 & 13.42 & 12.35 & 11.76 & 4.3 \\
\hline 214011.35 & +573951.8 & 17.65 & 16.15 & 16.61 & 14.31 & 13.44 & 12.97 & 7.1 \\
\hline 214021.30 & +572657.9 & 16.95 & 15.92 & 15.93 & 14.50 & 13.40 & 12.62 & 3.6 \\
\hline 6.90 & +581437.9 & 14.85 & 13.77 & 13.87 & 11.90 & 10.89 & 10.23 & 3.7 \\
\hline 2140 & +581 & 19.00 & 17.08 & 17.25 & 3.94 & 12.54 & 11.64 & 6.7 \\
\hline 214055.93 & +57 1759.2 & 17.78 & 16.16 & 16.06 & 13.81 & 12.83 & 12.24 & 7.3 \\
\hline 214115.97 & +580826.5 & 18.20 & 16.71 & 16.72 & 14.47 & 13.30 & 12.53 & 8.4 \\
\hline 214139.49 & +501749.8 & 17.84 & 16.93 & 16.76 & 15.41 & 14.72 & 14.33 & 29.8 \\
\hline 214202.30 & +544 & 19.36 & 17.86 & 18.04 & 15.29 & .97 & 3.06 & 20.7 \\
\hline 214217.63 & +565550.2 & 16.69 & 15.32 & 15.64 & 12.43 & 11.53 & 11.02 & 8.6 \\
\hline 214306.36 & +510610.6 & 18.12 & 17.63 & 17.29 & 15.07 & 14.15 & 13.54 & 28.0 \\
\hline 214418.19 & +472344.2 & 16.27 & 15.32 & 15.36 & 13.19 & 12.13 & 11.54 & 47.6 \\
\hline 21443 & +573732.7 & 17.98 & 16.54 & 16.94 & 14.34 & 13.44 & 12.86 & 14.3 \\
\hline 214441.34 & +574611.9 & 17.19 & 16.00 & 16.23 & 14.17 & 13.09 & 12.39 & 16.3 \\
\hline 214443.70 & +571419.8 & 18.14 & 17.33 & 17.13 & 35 & 15.05 & 13.80 & 12.6 \\
\hline 214547.74 & +564845.8 & 19.06 & 16.88 & 17.54 & 14.33 & 13.40 & 12.82 & 10.3 \\
\hline 214600.27 & +572309.6 & 17.15 & 15.72 & 16.06 & 12.65 & 11.46 & 10.63 & 10.9 \\
\hline 214625.99 & +572828.9 & 18.04 & 16.96 & 15.68 & 14.07 & 13.06 & 12.30 & 12.0 \\
\hline 214640.27 & +471327.6 & 17.25 & 15.84 & 16.07 & 13.37 & 12.25 & 11.50 & 50.2 \\
\hline 214811.78 & +575941.6 & 16.71 & 15.43 & 15.50 & 12.92 & 11.66 & 10.84 & 15.2 \\
\hline 214933.64 & +574502.2 & 16.70 & 15.35 & 15.66 & 13.14 & 12.43 & 11.86 & 17.4 \\
\hline 215331.40 & +562927.0 & 18.79 & 18.13 & 16.47 & 14.94 & 13.30 & 12.06 & 19.0 \\
\hline 215345.14 & +594342.0 & 18.25 & 16.84 & 17.12 & 14.82 & 13.91 & 13.35 & 13.9 \\
\hline 215447.24 & +531347.0 & 18.32 & 17.27 & 16.78 & 15.43 & 14.16 & 13.10 & 31.5 \\
\hline 215628.47 & +571445.5 & 15.14 & 14.38 & 13.48 & 12.97 & 11.83 & 10.31 & 24.5 \\
\hline 215812.70 & +522621.6 & 19.12 & 17.86 & 17.92 & 15.88 & 15.02 & 14.37 & 15.8 \\
\hline 215818.39 & +552631.9 & 19.31 & 18.08 & 17.87 & 15.71 & 14.42 & 13.64 & 10.6 \\
\hline 215820.72 & +552632.6 & 19.29 & 17.97 & 18.05 & 15.32 & 13.99 & 13.00 & 10.4 \\
\hline 215858.32 & +524603.0 & 19.17 & 18.37 & 17.68 & 16.47 & 15.43 & 14.63 & 13.4 \\
\hline 215936.96 & +552433.4 & 17.96 & 16.59 & 16.93 & 14.37 & 13.62 & 13.19 & 12.3 \\
\hline 220108.17 & +555441.4 & 16.14 & 15.36 & 14.99 & 13.03 & 11.62 & 10.43 & 11.0 \\
\hline 220210.21 & +584544.6 & 17.31 & 15.93 & 16.29 & 13.96 & 13.22 & 12.57 & 24.4 \\
\hline 220313.49 & +593957.2 & 17.62 & 16.20 & 16.43 & 13.85 & 12.88 & 12.29 & 7.5 \\
\hline 220330.08 & +582804.2 & 14.90 & 13.92 & 13.91 & 12.07 & 11.16 & 10.64 & 21.8 \\
\hline 220513.54 & +572553.5 & 19.10 & 18.04 & 17.56 & 15.12 & 14.09 & 13.55 & 22.4 \\
\hline 220609.07 & +604241.4 & 16.28 & 15.03 & 15.14 & 13.05 & 11.62 & 10.39 & 20.8 \\
\hline 220648.59 & +541147.5 & 18.67 & 17.42 & 17.22 & 16.48 & 15.44 & 14.46 & 20.3 \\
\hline 220715.51 & +55 1239.2 & 19.31 & 17.77 & 18.13 & 16.53 & 15.35 & 14.44 & 19.2 \\
\hline 220802.26 & +605905.6 & 17.92 & 16.50 & 16.51 & 15.13 & 14.16 & 13.55 & 7.5 \\
\hline 220943.15 & +585622.7 & 17.98 & 16.60 & 16.95 & 13.81 & 12.64 & 11.84 & 14.2 \\
\hline
\end{tabular}


Table 1. continued.

\begin{tabular}{|c|c|c|c|c|c|c|c|c|}
\hline \multicolumn{2}{|c|}{ RA (J2000.0) Dec } & $r$ & $i$ & $\mathrm{H} \alpha$ & $J$ & $\bar{H}$ & $\overline{K_{\mathrm{s}}}$ & $d_{4}$ \\
\hline 221100.10 & +605633.5 & 17.69 & 16.48 & 16.26 & 14.19 & 13.13 & 12.46 & 16.3 \\
\hline 221146.04 & +604203.6 & 17.25 & 16.22 & 16.07 & 14.32 & 13.42 & 12.81 & 9.3 \\
\hline 221154.94 & +585722.1 & 18.84 & 16.97 & 17.68 & 13.95 & 12.57 & 11.53 & 10.3 \\
\hline 221213.10 & +585202.8 & 17.34 & 16.30 & 15.65 & 13.41 & 11.96 & 10.83 & 10.4 \\
\hline 221232.53 & +604250.3 & 17.43 & 16.37 & 16.41 & 13.84 & 12.86 & 12.30 & 8.7 \\
\hline 221311.17 & +542531.4 & 17.80 & 17.64 & 15.89 & 16.59 & 15.14 & 14.19 & 26.6 \\
\hline 221316.57 & +561213.5 & 17.71 & 16.53 & 16.66 & 13.80 & 12.88 & 12.25 & 16.4 \\
\hline 221320.22 & +602929.2 & 18.04 & 16.74 & 17.03 & 14.69 & 13.49 & 12.84 & 8.3 \\
\hline 221334.04 & +6035 58.7 & 18.79 & 17.35 & 17.50 & 14.69 & 13.64 & 13.04 & 6.2 \\
\hline 221349.64 & +55 5407.3 & 19.08 & 18.34 & 17.57 & 15.97 & 15.02 & 14.32 & 15.0 \\
\hline 221351.53 & +604424.8 & 18.43 & 17.02 & 16.84 & 13.96 & 12.68 & 11.99 & 7.9 \\
\hline 221359.71 & +603506.2 & 16.40 & 15.30 & 15.45 & 12.98 & 11.94 & 11.35 & 6.0 \\
\hline 221411.56 & +612606.4 & 17.56 & 16.41 & 16.43 & 14.42 & 13.04 & 12.12 & 3.0 \\
\hline 221419.27 & +603806.7 & 19.32 & 18.04 & 17.96 & 15.62 & 14.33 & 13.27 & 5.9 \\
\hline 221428.89 & +612524.0 & 17.68 & 16.26 & 16.63 & 13.80 & 12.54 & 11.70 & 1.6 \\
\hline 221439.83 & +612558.8 & 17.69 & 16.20 & 16.57 & 13.65 & 12.50 & 11.81 & 1.2 \\
\hline 221440.31 & +612613.8 & 15.33 & 14.14 & 14.22 & 11.91 & 10.66 & 9.77 & 1.3 \\
\hline 221442.24 & +604402.5 & 19.43 & 17.57 & 17.79 & 15.23 & 14.31 & 13.84 & 7.3 \\
\hline 221508.95 & +610241.1 & 17.02 & 15.85 & 15.84 & 14.01 & 12.82 & 12.13 & 7.0 \\
\hline 221511.99 & +575251.4 & 17.83 & 17.08 & 16.14 & 14.98 & 13.47 & 11.97 & 14.3 \\
\hline 221518.87 & +582312.6 & 19.00 & 17.64 & 17.30 & 86 & .22 & 11.62 & 16.5 \\
\hline 221537.63 & +610655.1 & 16.81 & 15.61 & 15.66 & 13.48 & 12.50 & 11.89 & 4.3 \\
\hline 221602.80 & +561427.3 & 17.42 & 16.56 & 15.79 & 14.45 & 13.36 & 12.70 & 11.1 \\
\hline 221621.24 & +600630.4 & 18.58 & 17.47 & 17.59 & 15.14 & 13.94 & 12.78 & 28.5 \\
\hline 221639.34 & +604757.4 & 18.10 & 16.61 & 17.06 & 13.51 & 12.33 & 11.51 & 5.5 \\
\hline 221644.69 & +604 & 18.81 & 17.39 & 16.98 & .85 & .77 & 3.15 & 5.5 \\
\hline 221655.98 & +605343.7 & 17.93 & 16.40 & 16.33 & 13.57 & 12.19 & 11.27 & 7.3 \\
\hline 221734.33 & +611408.8 & 19.43 & 17.92 & 17.61 & 15.28 & 14.06 & 13.39 & 11.2 \\
\hline 221740.25 & +614702.5 & 17.73 & 16.30 & 16.15 & 12.83 & 11.50 & 10.52 & 11.5 \\
\hline 221755.42 & +610 & 18.67 & 17.01 & 17.36 & 69 & .70 & 13.13 & 12.2 \\
\hline 221808.49 & +560553.3 & 13.73 & 13.02 & 12.54 & 38 & 10.18 & 9.03 & 8.1 \\
\hline 221818.15 & +560609.7 & 17.78 & 16.64 & 16.72 & 14 & 13.52 & 12.80 & 7.2 \\
\hline 221825.73 & +613726.5 & 18.46 & 16.67 & 17.21 & 14.46 & 13.60 & 13.16 & 5.2 \\
\hline 221903.17 & +613756.4 & 15.70 & 14.65 & 14.69 & 12.98 & 11.79 & 11.11 & 4.0 \\
\hline 221911.20 & +605 & 18.86 & 17.20 & 17.42 & 13.78 & 11.74 & 10.20 & 12.5 \\
\hline 221911.45 & +560253.3 & 19.11 & 18.03 & 17.85 & 15.83 & 14.92 & 14.26 & 9.0 \\
\hline 221914.69 & +613847.5 & 18.34 & 16.99 & 17.14 & 14.60 & 13.87 & 13.34 & 4.8 \\
\hline 22205 & +5848 & 15.46 & 14.10 & 14.30 & 11.29 & 9.84 & 8.63 & 19.1 \\
\hline 22254 & +612 & 19.20 & 17.65 & 17.77 & 14.77 & 13.80 & 13.14 & 24.5 \\
\hline 222700.27 & +561732.5 & 16.51 & 15.64 & 15.12 & 13.78 & 12.86 & 12.11 & 23.6 \\
\hline 222800.09 & +630908.9 & 16.96 & 15.64 & 15.96 & 13.50 & 12.51 & 11.86 & 17.0 \\
\hline 223150.40 & +591246.0 & 16.09 & 14.80 & 15.01 & 12.77 & 11.54 & 10.85 & 19.8 \\
\hline 223151.98 & +6310 & 19.09 & 17.14 & 17.42 & 14.62 & 13.68 & 13.17 & 27.8 \\
\hline 223645.28 & +615252.2 & 19.50 & 17.64 & 17.72 & 14.70 & 13.49 & 12.80 & 21.7 \\
\hline 223703.73 & +585849.4 & 16.20 & 14.55 & 15.12 & 12.57 & 11.79 & 11.40 & 18.3 \\
\hline 223728.63 & +583753.1 & 18.53 & 16.17 & 16.76 & 13.33 & 12.43 & 11.88 & 19.6 \\
\hline 223819.84 & +621212.1 & 16.71 & 15.68 & 15.44 & 13.83 & 12.55 & 11.50 & 19.8 \\
\hline 223853.88 & +615628.9 & 16.72 & 15.48 & 15.60 & 13.52 & 12.43 & 11.69 & 22.6 \\
\hline 224055.96 & +613109.3 & 19.43 & 17.86 & 17.49 & 15.31 & 14.34 & 13.66 & 31.4 \\
\hline 224214.74 & +594952.6 & 17.91 & 16.82 & 16.98 & 14.94 & 14.15 & 13.73 & 29.4 \\
\hline 224413.21 & +610045.1 & 18.32 & 16.95 & 17.11 & 14.37 & 13.25 & 12.45 & 19.3 \\
\hline 224508.11 & +591123.0 & 18.30 & 16.66 & 17.17 & 13.98 & 12.44 & 11.11 & 26.8 \\
\hline 224518.08 & +613049.9 & 17.37 & 16.19 & 15.67 & 14.32 & 13.36 & 12.74 & 21.5 \\
\hline 224638.35 & +610809.0 & 17.03 & 15.79 & 15.46 & 13.83 & 12.84 & 12.28 & 10.5 \\
\hline 224714.25 & +592149.1 & 17.22 & 15.69 & 15.78 & 12.40 & 10.78 & 9.53 & 15.8 \\
\hline 224722.39 & +580121.5 & 14.00 & 12.95 & 12.85 & 10.80 & 9.76 & 8.85 & 8.3 \\
\hline 224906.90 & +620734.8 & 16.89 & 15.54 & 15.82 & 13.06 & 11.96 & 11.22 & 14.3 \\
\hline 224908.54 & +612406.8 & 19.40 & 18.07 & 17.88 & 15.53 & 14.48 & 13.83 & 15.7 \\
\hline 225250.89 & +623617.3 & 18.45 & 17.17 & 17.22 & 14.92 & 13.70 & 12.97 & 5.8 \\
\hline 225326.79 & +623531.7 & 18.43 & 16.58 & 17.07 & 13.85 & 12.75 & 12.14 & 3.1 \\
\hline 225331.30 & +62 3711.4 & 18.31 & 16.68 & 16.73 & 14.01 & 12.84 & 12.11 & 2.5 \\
\hline
\end{tabular}


Table 1. continued.

\begin{tabular}{|c|c|c|c|c|c|c|c|c|}
\hline \multicolumn{2}{|c|}{ RA (J2000.0) Dec } & $r$ & $i$ & $\mathrm{H} \alpha$ & $\bar{J}$ & $\bar{H}$ & $\overline{K_{\mathrm{s}}}$ & $d_{4}$ \\
\hline 225350.55 & +613246.4 & 17.93 & 16.48 & 16.82 & 14.23 & 13.15 & 12.43 & 22.8 \\
\hline 225351.96 & +623435.7 & 18.42 & 16.78 & 16.57 & 14.24 & 13.09 & 12.38 & 3.0 \\
\hline 225352.02 & +623809.0 & 16.67 & 15.42 & 15.62 & 13.36 & 12.15 & 11.37 & 1.5 \\
\hline 225352.17 & +623746.3 & 18.39 & 16.81 & 17.02 & 14.02 & 12.91 & 12.20 & 1.4 \\
\hline 225407.57 & +623851.5 & 18.48 & 16.72 & 17.06 & 13.55 & 12.17 & 11.25 & 2.1 \\
\hline 225409.88 & +625742.9 & 18.56 & 17.35 & 17.47 & 14.35 & 13.27 & 12.58 & 12.2 \\
\hline 225437.74 & +622930.8 & 18.37 & 16.78 & 16.85 & 14.08 & 12.96 & 12.37 & 7.3 \\
\hline 225452.08 & +615903.0 & 18.94 & 17.12 & 17.50 & 14.09 & 12.75 & 11.98 & 23.7 \\
\hline 225509.51 & +624123.2 & 17.87 & 16.38 & 16.07 & 13.87 & 12.70 & 11.97 & 3.0 \\
\hline 225521.80 & +623753.5 & 15.96 & 14.81 & 14.71 & 12.69 & 11.38 & 10.44 & 2.5 \\
\hline 225522.50 & +622825.2 & 17.59 & 16.33 & 16.43 & 14.19 & 13.07 & 12.33 & 7.9 \\
\hline 225526.19 & +623934.0 & 19.31 & 17.26 & 17.75 & 14.57 & 13.66 & 13.06 & 2.6 \\
\hline 225536.82 & +621115.8 & 18.08 & 16.67 & 16.84 & 14.66 & 13.31 & 12.32 & 17.6 \\
\hline 225537.20 & +630004.9 & 18.95 & 17.34 & 17.33 & 14.46 & 13.17 & 12.24 & 6.7 \\
\hline 225538.17 & +623606.3 & 19.13 & 17.45 & 17.80 & 15.05 & 13.76 & 12.85 & 2.2 \\
\hline 225539.41 & +623739.0 & 19.06 & 16.93 & 17.33 & 14.08 & 12.97 & 12.29 & 1.9 \\
\hline 225552.04 & +623652.1 & 18.52 & 17.25 & 16.99 & 15.08 & 13.97 & 13.33 & 1.9 \\
\hline 225553.65 & +58 1431.3 & 18.44 & 17.28 & 16.32 & 14.98 & 13.90 & 13.33 & 20.3 \\
\hline 225558.51 & +584057.9 & 17.66 & 15.73 & 16.37 & 13.09 & 12.37 & 11.93 & 12.2 \\
\hline 225559.58 & +623757.5 & 17.95 & 16.83 & 16.44 & 14.82 & 13.95 & 13.34 & 2.3 \\
\hline 225602.82 & +624513.8 & 16.85 & 15.57 & 15. & 34 & .28 & .63 & 2.0 \\
\hline 225613.18 & +623533.1 & 15.24 & 14.04 & 14.19 & 11.80 & 10.78 & 10.12 & 3.4 \\
\hline 225617.55 & +584421.5 & 17.54 & 16.38 & 16.20 & 13.93 & 12.58 & 11.63 & 12.6 \\
\hline 225623.13 & +624354.2 & 18.76 & 17.00 & 17.38 & 14.45 & 13.32 & 12.68 & 2.0 \\
\hline 225626.03 & +623900.9 & 17.57 & 16.11 & 16.39 & 13.61 & 12.39 & 11.73 & 2.6 \\
\hline 225628.04 & +630834.1 & 19.24 & 17.89 & 17.87 & .61 & .51 & 13.68 & 10.2 \\
\hline 225630.87 & +624215.7 & 19.10 & 16.69 & 17.26 & 13.48 & 12.50 & 11.92 & 2.0 \\
\hline 225630.91 & +582447.4 & 18.26 & 16.60 & 17.03 & 13.57 & 11.95 & 10.66 & 14.1 \\
\hline 225712.85 & +594648.2 & 18.05 & 16.75 & 16.98 & 14.51 & 13.37 & 12.70 & 11.1 \\
\hline 225725.85 & +625629.0 & 19.34 & 17.80 & 17.76 & .53 & .54 & 3.01 & 11.6 \\
\hline 225906.10 & +624630.0 & 17.55 & 16.41 & 15.83 & 14.32 & 13.21 & 12.56 & 10.6 \\
\hline 230034.78 & +623436.3 & 18.56 & 17.01 & 17.13 & 14.86 & 13.37 & 12.47 & 13.4 \\
\hline 230137.10 & +612613.3 & 18.06 & 16.58 & 16.82 & 13.50 & 12.01 & 10.99 & 6.9 \\
\hline 1.49 & +614054.2 & 18.11 & 16.54 & 16.99 & 14.62 & 13.76 & 13.26 & 12.9 \\
\hline 230314.95 & +631 & 18.63 & 17.52 & 17.27 & 16.02 & 4.46 & 13.24 & 9.7 \\
\hline 230327.99 & +623106.8 & 17.09 & 15.43 & 15.90 & 12.52 & 11.15 & 10.29 & 19.1 \\
\hline 230342.17 & +611850.4 & 17.55 & 15.66 & 15.52 & 13.04 & 11.81 & 11.12 & 16.6 \\
\hline 46.02 & +6148 & 14.61 & 13.50 & 13.54 & 11.36 & 10.18 & 9.33 & 4.6 \\
\hline 03.38 & +615 & 16.35 & 15.18 & 15.13 & 3.01 & .79 & 10.97 & 6.3 \\
\hline 230412.45 & +593954.0 & 17.92 & 16.55 & 16.90 & 54 & 13.40 & 12.79 & 17.5 \\
\hline 230413.56 & +595727.0 & 18.47 & 17.29 & 17.34 & 15.26 & 14.20 & 13.57 & 16.2 \\
\hline 230439.95 & +62 2619.8 & 18.42 & 16.77 & 17.27 & 14.33 & 13.02 & 12.22 & 16.4 \\
\hline 230602.00 & +60 1238.7 & 19.27 & 18.31 & 17.81 & 16.23 & 15.41 & 14.73 & 6.8 \\
\hline 230638.25 & +623204.1 & 16.35 & 14.89 & 15.24 & 12.25 & 10.93 & 9.99 & 14.5 \\
\hline 230756.11 & +622414.4 & 19.41 & 17.20 & 17.65 & 14.26 & 13.41 & 12.90 & 12.0 \\
\hline 230818.22 & +583414.9 & 19.43 & 18.35 & 18.51 & 16.44 & 15.58 & 15.13 & 11.7 \\
\hline 230845.42 & +622712.5 & 19.05 & 17.09 & 17.43 & 14.34 & 13.30 & 12.68 & 9.8 \\
\hline 230847.33 & +582507.5 & 18.25 & 17.14 & 17.23 & 15.00 & 13.97 & 13.22 & 13.6 \\
\hline 231047.31 & +623628.7 & 18.62 & 16.76 & 17.02 & 14.05 & 12.88 & 12.23 & 17.4 \\
\hline 231142.83 & +660011.3 & 17.84 & 15.99 & 16.64 & 13.66 & 12.81 & 12.28 & 48.5 \\
\hline 231159.45 & +595326.5 & 19.10 & 18.01 & 17.96 & 15.95 & 14.71 & 13.90 & 11.4 \\
\hline 231201.75 & +634105.7 & 18.50 & 16.88 & 17.37 & 13.91 & 12.12 & 10.79 & 38.5 \\
\hline 231203.04 & +601838.6 & 17.68 & 16.13 & 16.44 & 13.06 & 11.39 & 10.15 & 14.4 \\
\hline 231204.71 & +624600.3 & 15.23 & 14.01 & 14.18 & 11.92 & 11.20 & 10.65 & 24.9 \\
\hline 231223.26 & +614318.4 & 18.08 & 16.33 & 16.67 & 13.98 & 13.05 & 12.53 & 9.5 \\
\hline 231307.09 & +590247.9 & 14.49 & 13.66 & 13.62 & 11.79 & 10.54 & 9.39 & 19.4 \\
\hline 231319.53 & +595016.2 & 18.55 & 17.34 & 17.59 & 15.05 & 14.11 & 13.31 & 7.7 \\
\hline 231526.39 & +642225.2 & 18.48 & 17.15 & 17.32 & 14.61 & 13.59 & 12.83 & 32.1 \\
\hline 231556.90 & +614516.9 & 17.75 & 16.74 & 16.22 & 14.82 & 13.56 & 12.63 & 17.2 \\
\hline 231610.45 & +621303.4 & 19.38 & 17.68 & 17.65 & 15.35 & 14.58 & 13.93 & 6.7 \\
\hline 231640.86 & +62 2057.1 & 16.78 & 15.68 & 15.82 & 14.15 & 12.86 & 11.89 & 5.8 \\
\hline
\end{tabular}


R. L. M. Corradi et al.: IPHAS and the symbiotic stars. I., Online Material p 20

Table 1. continued.

\begin{tabular}{rrrrrrrrrr}
\hline \hline \multicolumn{2}{c}{ RA (J2000.0) Dec } & $r$ & $i$ & $\mathrm{H} \alpha$ & $J$ & $H$ & $K_{\mathrm{s}}$ & $d_{4}$ \\
\hline 231735.92 & +634506.4 & 16.08 & 14.62 & 14.30 & 12.20 & 10.72 & 9.39 & 17.3 \\
231826.54 & +595346.6 & 19.18 & 18.10 & 18.11 & 16.10 & 14.92 & 14.15 & 19.4 \\
231836.35 & +640406.5 & 17.68 & 16.43 & 16.46 & 13.92 & 12.65 & 11.57 & 18.0 \\
232005.35 & +621841.7 & 17.59 & 16.22 & 16.32 & 13.91 & 12.81 & 12.19 & 20.8 \\
232227.11 & +590311.3 & 19.17 & 18.05 & 18.20 & 16.19 & 15.33 & 14.77 & 20.3 \\
232408.30 & +591752.9 & 19.45 & 18.12 & 18.12 & 15.93 & 14.85 & 13.97 & 19.4 \\
232640.12 & +621858.1 & 15.78 & 14.81 & 14.77 & 13.13 & 12.18 & 11.47 & 17.8 \\
232907.04 & +593430.4 & 17.36 & 15.79 & 16.07 & 12.41 & 10.70 & 9.43 & 24.3 \\
232937.83 & +621307.3 & 17.85 & 16.32 & 15.85 & 14.10 & 13.34 & 12.84 & 11.8 \\
233035.06 & +595526.0 & 19.15 & 17.69 & 17.71 & 15.36 & 14.34 & 13.74 & 28.9 \\
233244.44 & +614410.8 & 17.48 & 16.32 & 16.35 & 14.17 & 13.32 & 12.67 & 12.9 \\
233542.67 & +621228.5 & 18.18 & 16.48 & 17.08 & 14.44 & 13.58 & 13.15 & 15.6 \\
233618.62 & +623708.9 & 17.76 & 16.57 & 16.76 & 14.82 & 13.68 & 13.03 & 13.6 \\
233924.79 & +615940.9 & 18.57 & 16.89 & 17.37 & 14.34 & 13.08 & 12.18 & 10.8 \\
234039.28 & +613545.2 & 18.45 & 17.14 & 17.15 & 14.88 & 13.76 & 13.13 & 16.2 \\
234125.21 & +654042.6 & 15.03 & 13.39 & 13.55 & 10.50 & 8.82 & 7.45 & 50.9 \\
234133.78 & +635323.4 & 16.06 & 14.52 & 14.94 & 12.31 & 11.13 & 10.44 & 27.8 \\
234658.61 & +590143.2 & 17.32 & 16.19 & 16.23 & 14.38 & 13.30 & 12.54 & 18.8 \\
234732.55 & +632346.3 & 16.80 & 15.05 & 15.57 & 12.76 & 11.83 & 11.26 & 6.5 \\
234835.75 & +663053.4 & 15.71 & 14.71 & 14.67 & 12.95 & 11.91 & 11.29 & 44.3 \\
235105.38 & +625203.8 & 18.80 & 17.56 & 17.81 & 15.53 & 14.52 & 13.95 & 11.9 \\
235305.89 & +602112.7 & 19.27 & 17.51 & 17.99 & 14.41 & 13.01 & 12.03 & 23.4 \\
235609.52 & +655723.7 & 18.46 & 16.53 & 17.04 & 13.92 & 13.07 & 12.46 & 23.5 \\
235657.56 & +583034.9 & 16.75 & 14.64 & 15.22 & 12.18 & 11.31 & 10.83 & 29.0 \\
235727.29 & +582842.1 & 17.76 & 16.65 & 15.81 & 13.30 & 11.92 & 11.01 & 29.6 \\
235734.77 & +644649.1 & 14.43 & 13.44 & 13.47 & 11.49 & 10.55 & 9.88 & 20.6 \\
235812.52 & +625434.9 & 18.47 & 17.39 & 17.42 & 15.52 & 14.65 & 14.00 & 19.9 \\
235919.98 & +662312.0 & 14.63 & 13.32 & 13.32 & 11.06 & 9.82 & 8.99 & 29.9 \\
\hline
\end{tabular}

